\title{
Minimal Obstructions for Partial Representations of Interval Graphs*
}

\author{
Pavel Klavík ${ }^{\dagger}$ \\ Computer Science Institute \\ Charles University in Prague \\ Czech Republic \\ klavik@iuuk.mff.cuni.cz
}

\author{
Maria Saumell ${ }^{\ddagger}$ \\ Department of Theoretical Computer Science \\ Faculty of Information Technology \\ Czech Technical University in Prague; and \\ Institute of Computer Science \\ The Czech Academy of Sciences \\ Czech Republic \\ maria.saumell@fit.cvut.cz
}

Submitted: Jan 12, 2016; Accepted: Aug 24, 2018; Published: Dec 21, 2018

(C) The authors. Released under the CC BY-ND license (International 4.0).

\begin{abstract}
Interval graphs are intersection graphs of closed intervals. A generalization of recognition called partial representation extension was introduced recently. The input gives an interval graph with a partial representation specifying some predrawn intervals. We ask whether the remaining intervals can be added to create an extending representation. Two linear-time algorithms are known for solving this problem.

In this paper, we characterize the minimal obstructions which make partial representations non-extendible. This generalizes Lekkerkerker and Boland's characterization of the minimal forbidden induced subgraphs of interval graphs. Each minimal obstruction consists of a forbidden induced subgraph together with at most four pre-drawn intervals. A Helly-type result follows: A partial representation is extendible if and only if every quadruple of pre-drawn intervals is extendible by itself. Our characterization leads to a linear-time certifying algorithm for partial representation extension.
\end{abstract}

Mathematics Subject Classifications: 05C62, 68R10

\footnotetext{
*The conference version of this paper appeared in ISAAC 2014 [27].

†Supported by CE-ITI (P202/12/G061 of GAČR) and Charles University as GAUK 196213. A part of this work was carried out during a visit at ULB in Brussels, funded by a EUROCORES Short Term Visit grant.

${ }^{\ddagger}$ Supported by the Czech Science Foundation, grant number GJ16-07822Y, by institutional support RVO:67985807, by project LO1506 of the Czech Ministry of Education, Youth and Sports, by project NEXLIZ - CZ.1.07/2.3.00/30.0038 co-financed by European Social Fund and the state budget of Czech Republic, and by ESF EuroGIGA project ComPoSe as F.R.S.-FNRS - EUROGIGA NR 13604.
} 
For an interactive structural diagram of this paper, see the following website (supported for Firefox and Google Chrome):

$$
\text { http://pavel.klavik.cz/orgpad/minobstr.html }
$$

\section{Introduction}

The main motivation for graph drawing and geometric representations is finding ways to visualize some given data efficiently. The most famous representations are plane drawings, in which we draw a graph in the plane and we want to avoid (or minimize) crossings of edges. However, for certain types of graphs, intersection representations are more suitable. They represent each vertex by a geometrical object and encode the edges by intersections.

\section{$1.1 \quad$ Interval Graphs}

The most studied class of intersection graphs are interval graphs (INT), defined by Hájos [15] in 1957. An interval representation $\mathcal{R}$ is a collection of closed intervals $\{\langle x\rangle: x \in V(G)\}$ where $\langle x\rangle \cap\langle y\rangle \neq \emptyset$ if and only if $x y \in E(G)$. A graph is an interval graph if it has an interval representation; see Fig. 1a.

Interval graphs have many applications. Already in 1959, Benzer [4] used them in his experimental study of DNA. For some time, interval graphs played an important role for the DNA hybridization [18], in which short pieces of DNA are studied independently. Further applications include scheduling, psychology, archaeology, etc. [39, 35, 19].

Interval graphs also have nice theoretical properties. They are perfect and closely related to path-width decompositions. They can be recognized in linear time $[6,11,32]$, and many hard combinatorial problems are polynomially solvable for interval graphs. Fulkerson and Gross [14] characterized them by consecutive orderings of maximal cliques (see Section 3 for details). This lead Booth and Lueker [6] to the construction of PQ-trees, which are an efficient data structure to deal with consecutive orderings, and have many other applications.

Chordal graphs (CHOR) are graphs with no induced cycle of length four or more, alternatively intersection graphs of subtrees of trees. Three vertices form an asteroidal triple if there exists a path between every pair of them avoiding the neighborhood of the third vertex. Asteroidal triple-free graphs (AT-FREE) are graphs containing no asteroidal triples. Lekkerkerker and Boland [30] characterized interval graphs as INT $=\mathrm{CHOR} \cap \mathrm{AT}$-FREE.

(a)

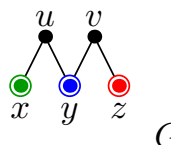

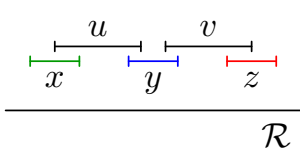

(b)

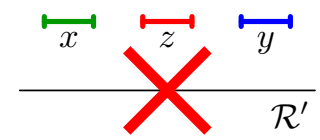

Figure 1: (a) An interval graph $G$ with one of its interval representations $\mathcal{R}$. (b) A partial representation $\mathcal{R}^{\prime}$ with pre-drawn intervals $\langle x\rangle^{\prime},\langle y\rangle^{\prime}$ and $\langle z\rangle^{\prime}$. It is non-extendible since $\langle u\rangle$ cannot be placed. In all figures, we depict pre-drawn intervals in bold. 

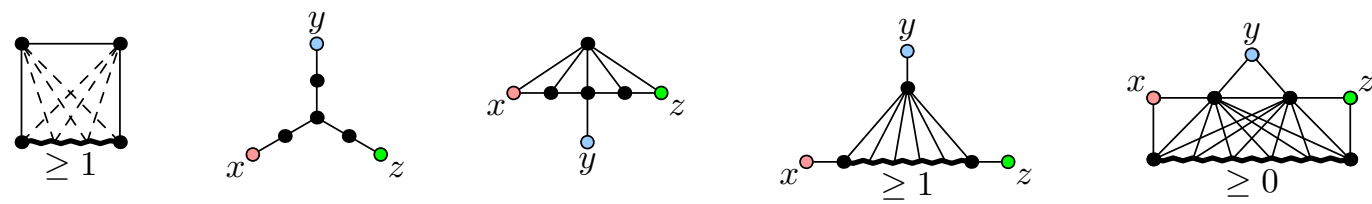

Figure 2: Five types of LB obstructions which are the minimal forbidden induced subgraphs of INT. The bold curly lines correspond to induced paths with denoted minimal lengths. The leftmost obstructions are induced cycles of length four or more. The remaining four types of obstructions are minimal asteroidal triples $(x, y, z)$ which are chordal graphs.

They described this characterization by the minimal forbidden induced subgraphs given in Fig. 2 which we call Lekkerkerker-Boland obstructions (LB).

\subsection{Partial Representation Extension}

The partial representation extension problem was introduced by Klavík et al. [24]. A partial representation $\mathcal{R}^{\prime}$ of $G$ is an interval representation $\left\{\langle x\rangle^{\prime}: x \in V\left(G^{\prime}\right)\right\}$ of an induced subgraph $G^{\prime}$ of $G$. The vertices of $G^{\prime}$ and the intervals of $\mathcal{R}^{\prime}$ are called pre-drawn. A representation $\mathcal{R}$ of $G$ extends $\mathcal{R}^{\prime}$ if and only if it assigns the same intervals to the vertices of $G^{\prime}$, i.e., $\langle x\rangle=\langle x\rangle^{\prime}$ for every $x \in V\left(G^{\prime}\right)$. For an example, see Fig. 1b.

\section{Problem: Partial Representation Extension - REPExT(INT) \\ Input: $\quad$ A graph $G$ and a partial representation $\mathcal{R}^{\prime}$ of $G^{\prime}$. \\ Output: Is there an interval representation of $G$ extending $\mathcal{R}^{\prime}$ ?}

The first polynomial-time algorithm, running in $\mathcal{O}\left(n^{2}\right)$ time, was given in [24]. Currently, there are two different linear-time algorithms [5, 23] for this problem.

We note that the partial representation extension problems have been considered also for other classes of intersection graphs. A linear-time algorithm for proper interval graphs and an almost quadratic-time algorithm for unit interval graphs are given in [21], and improved to quadratic time in $[37,38]$. The partial representation extension problems are polynomial-time solvable for $k$-nested interval graphs (classes generalizing proper interval graphs), but NP-hard for $k$-length interval graphs (classes generalizing unit interval graphs), even for $k=2[25,26]$. Polynomial-time algorithms are further known for circle graphs [8], permutation and function graphs [20], proper circular-arc graphs [3], and trapezoid graphs [29]. The partial representation extension problems for chordal graphs [22], unit circular-arc graphs [40], and contact representations of planar graphs [7] are NP-hard. The complexity of the partial representation extension problem is open for circular-arc graphs.

Outside intersection graphs, a similar problem was considered even sooner for planar graphs. Partially embedded planar graphs can be extended in linear time [1]. Even though every planar graph has a straight-line embedding, extension of such embeddings 
is NP-hard [33]. Kuratowski's characterization of minimal forbidden minors was extended to partially embedded planar graphs by Jelínek et al. [17]. Our research has a similar spirit as this last result. Also, partial representation extension problem was studied for visibility representations [9].

\subsection{Our Results}

In this paper, we generalize the characterization of Lekkerkerker and Boland [30] to describe minimal obstructions which make partial representations non-extendible. Each obstruction consists of a small graph and its non-extendible partial representation. Aside LB obstructions, we have two trivial obstructions, called SE, and ten infinite classes of minimal obstructions. The main class, called $k$-FAT obstructions, has three wrongly ordered disjoint pre-drawn intervals $\left\langle x_{k}\right\rangle^{\prime},\left\langle y_{k}\right\rangle^{\prime}$, and $\left\langle z_{k}\right\rangle^{\prime}$. The obstruction consists of a zig-zag structure with $k$ levels where the last level cannot be placed. See Fig. 3a and b for 1-FAT and 2-FAT obstructions. There are eight other infinite classes derived from $k$-FAT obstructions by adding a few vertices and having different vertices pre-drawn. The last infinite class of $(k, \ell)$-CE obstructions consists of a $k$-FAT obstruction glued with an $\ell$-FAT obstruction and contains only two pre-drawn vertices; see Fig. 3c for a $(1,1)$-CE obstruction. We formally define these minimal obstructions in Section 2.

Theorem 1. A partial representation $\mathcal{R}^{\prime}$ of $G$ is extendible if and only if $G$ and $\mathcal{R}^{\prime}$ contain no LB, SE, $k$-FAT, $k$-BI, $k$-FS, $k$-EFS, $k$-FB, $k$-EFB, $k$-FDS, $k$-EFDS, $k$-FNS and $(k, \ell)-C E$ obstructions.

Since every minimal obstruction contains at most four pre-drawn intervals, we get the following Helly-type result as a straightforward corollary:

Corollary 2. A partial representation is extendible if and only if every quadruple of pre-drawn intervals is extendible by itself.

All known algorithms for the partial representation extension problems [24, 20, 5, 23, $21,22]$ are able to certify solvable instances by outputting an extending representation.
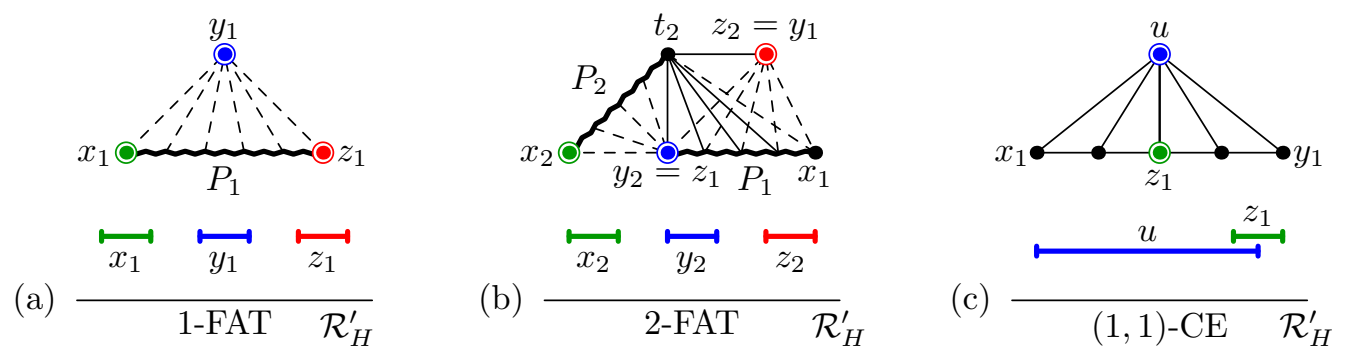

Figure 3: Three examples of minimal obstructions, each consisting of a graph $H$ and a non-extendible partial representation $\mathcal{R}_{H}^{\prime}$. Curly lines denote induced paths and dashed edges are non-edges. The obstructions (a) and (b) are the first two $k$-FAT obstructions, and $(\mathrm{c})$ is the simplest $(k, \ell)$-CE obstruction. 
Using our minimal obstructions, we construct the first algorithm for partial representation extension certifying also non-extendible partial representations. ${ }^{1}$

Corollary 3. Assume that the input gives the endpoints in a partial representation sorted from left to right. Then there exists an $\mathcal{O}(n+m)$ certifying algorithm for the partial representation extension problem, where $n$ is the number of vertices and $m$ is the number of edges of the input graph. If the answer is "yes", it outputs an extending representation. If the answer is "no", it detects one of the minimal obstructions.

Outline. In Section 2, we define the minimal obstructions which make a partial representation non-extendible. In Section 3, we introduce the standard tools for working with interval graphs: the characterization of Fulkerson and Gross by linear orderings of maximal cliques, and the related data structure called MPQ-tree which stores all feasible orderings.

In Section 4, we restate the characterization of [23]: a partial representation is extendible if and only if there exists a feasible ordering of the maximal cliques which extends a certain partial ordering $\triangleleft$. Therefore, to solve RePExT(INT), we test whether the MPQ-tree can be reordered according $\triangleleft$.

Based on this, in Section 5, we build our strategy for showing that every non-extendible partial representation contains one of the defined minimal obstructions. If reordering of the MPQ-tree according to $\triangleleft$ fails, then it fails in a leaf, in a P-node, or a Q-node. We deal with these three cases in Sections 6, 7, and 8, respectively, where the last one is most involved. In Section 9, we put these results together and establish Theorem 1 and Corollaries 2 and 3.

We conclude with a discussion of our results and several open problems.

\subsection{Preliminaries}

For a graph $G$, we denote by $V(G)$ its vertices and by $E(G)$ its edges. We denote the closed neighborhood of $x$ by $N[x]$. Maximal cliques are denoted by the letters $a$ to $f$, and vertices by the remaining letters. For $A \subseteq V(G)$, we denote by $G[A]$ the subgraph induced by $A$. Similarly, for $A \subseteq V\left(G^{\prime}\right)$, we denote by $\mathcal{R}^{\prime}[A]$ the partial representations which only contains the pre-drawn intervals in $A$. By $P_{x, y}$ we denote an induced path from $x$ to $y$; its length is the number of edges.

For an interval $\langle x\rangle$, we denote its left endpoint by $\ell(x)$ and its right endpoint by $r(x)$. If $r(x)\langle\ell(y)$, we say that $\langle x\rangle$ is on the left of $\langle y\rangle$ and $\langle y\rangle$ is on the right of $\langle x\rangle$. We say that $\langle y\rangle$ is between $\langle x\rangle$ and $\langle z\rangle$ if $\langle x\rangle$ is on the left of $\langle y\rangle$ and $\langle z\rangle$ is on the right of $\langle y\rangle$, or vice versa. We also work with open intervals, for which the inequalities are non-strict.

We conclude with a list of the remaining notation. In Section 3, we define MPQ-trees, $s(N), s_{i}(Q), s_{u}^{\leftarrow}(Q), s_{u}(Q), G[T], G[N], T[N]$, and $Q$-monotone paths. In Section 4, we define $\curvearrowleft(a), \curvearrowright(a), I_{a}, \triangleleft$, the flip operation, $P^{\mapsto}(a)$, and $P^{\leftrightarrow}(a)$.

\footnotetext{
${ }^{1}$ Formally speaking, a polynomial-time algorithm certifies unsolvable instances by outputting "no" and by a proof of its correctness. Our algorithm outputs a simple proof that a given partial representation is non-extendible in terms of a minimal obstruction. This proof can be independently verified which is desirable.
} 


\section{Definition of Minimal Obstructions}

In this section, we formally define all twelve classes of minimal obstructions which make a partial representation non-extendible. Since the list is long, the description is quite technical. In the rest of the paper, we prove that the list is complete.

Definition. Every obstruction consists of a graph $H$ and a non-extendible partial representation $\mathcal{R}_{H}^{\prime}$. This obstruction is contained in $G$ and $\mathcal{R}^{\prime}$ if the following holds:

(i) There exists an injective mapping $\varphi: V(H) \rightarrow V(G)$ such that $u v \in E(H)$ if and only if $\varphi(u) \varphi(v) \in E(G)$. So $\varphi(H)$ is an induced subgraph of $G$.

(ii) The pre-drawn vetices of $H$ are mapped by $\varphi$ to pre-drawn vertices of $G$.

(iii) The endpoints in $\mathcal{R}_{H}^{\prime}$ have the same left-to-right order as the endpoints of the corresponding pre-drawn vertices in $\mathcal{R}^{\prime}$.

For an example, see Fig. 4. If $G$ and $\mathcal{R}^{\prime}$ contain an obstruction, then $\mathcal{R}^{\prime}$ is clearly nonextendible.

We give $H$ by descriptions using finitely many vertices, edges, and induced paths. For inner vertices of the induced paths, we specify their adjacencies with the remainder of $H$. Since these induced paths do not have fixed lengths, each description having at least one induced path defines an infinite class of forbidden subgraphs $H$. Unlike LB obstructions, most classes of minimal obstructions need infinitely many different descriptions. For instance, each FAT obstruction has $k$ induced paths, and different values of $k$ need different descriptions.

If $H$ contains an induced path $P_{x, y}$, and $x$ and $y$ are allowed to be adjacent, then $P_{x, y}$ can be a single edge. When $N[x]=N[y]$, we allow the length of $P_{x, y}$ to be zero, i.e., $x=y$.

Minimality. An obstruction is minimal if $\mathcal{R}_{H}^{\prime}$ becomes extendible when any vertex is removed or any pre-drawn interval is made free by removing it from the partial representation $\mathcal{R}_{H}^{\prime}$.
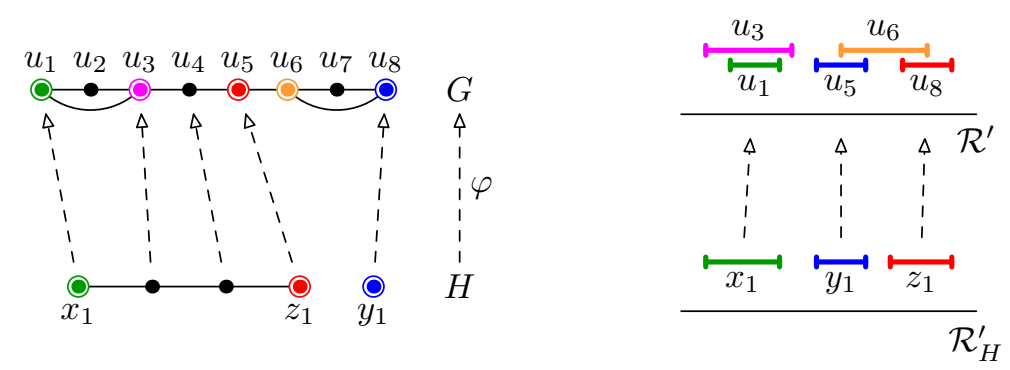

Figure 4: On top, an interval graph $G$ with a non-extendible partial representation. This is certified by containing a 1-FAT obstruction $H$ and $\mathcal{R}_{H}^{\prime}$, depicted on bottom. Notice that the preimage of $u_{3}$ is not predrawn and nothing is mapped to $u_{6}$, which means that $\mathcal{R}^{\prime}$ is non-extendible even when we free both $\left\langle u_{3}\right\rangle^{\prime}$ and $\left\langle u_{6}\right\rangle^{\prime}$. 

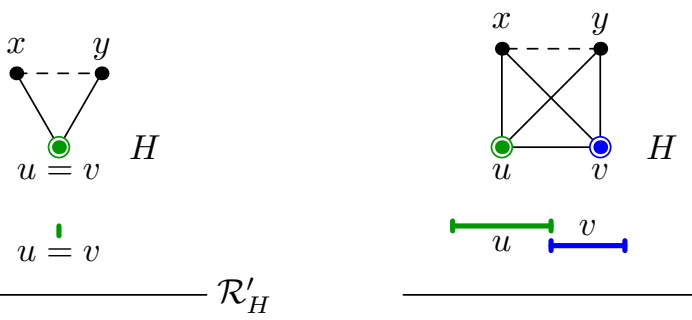

$\boldsymbol{R}_{H}^{\prime}$

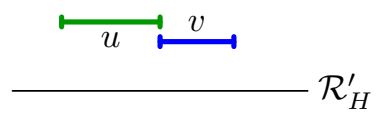

Figure 5: The SE obstructions, on the left with $u=v$, on the right with $u \neq v$.

\subsection{List of Minimal Obstructions}

In Fig. 2, we have already described minimal LB obstructions of [30] with $\mathcal{R}_{H}^{\prime}=\emptyset$. There are eleven other classes of minimal obstructions we describe now. To understand most of the paper, the reader should carefully read the definition of $k$-FAT obstructions (and possibly $k$-BI obstructions), while the definitions of the remaining classes can be checked when they appear.

SE obstructions. We start with two simple shared endpoint obstructions which deal

(a)

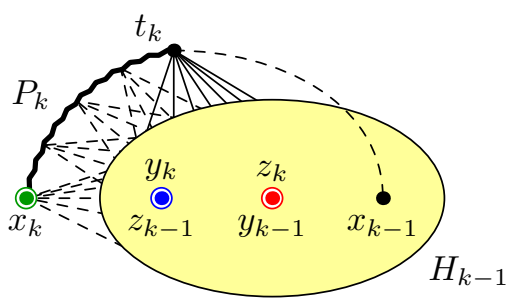

(b)

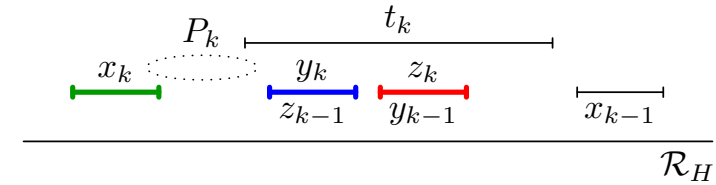

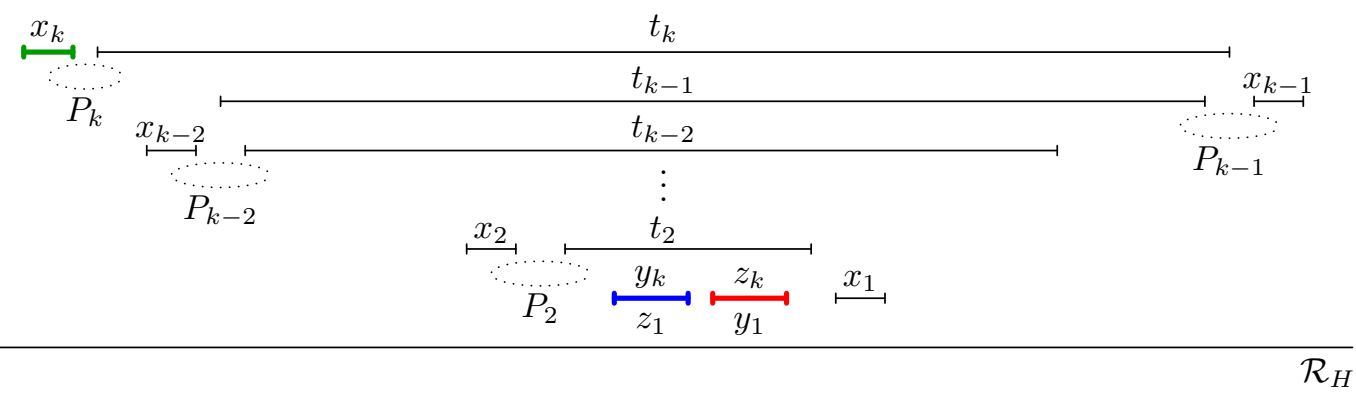

Figure 6: (a) A $k$-FAT obstruction is created from a $(k-1)$-FAT obstruction. It consists of the vertices $x_{1}, \ldots, x_{k}, t_{2}, \ldots, t_{k}, y_{k}, z_{k}$, and the induced paths $P_{1}, \ldots, P_{k}$. The adjacencies are defined inductively.

(b) In every representation $\mathcal{R}_{H}$, the pre-drawn interval $\left\langle x_{k}\right\rangle^{\prime}$ together with $P_{k}$ and $t_{k}$ forces $\left\langle x_{k-1}\right\rangle$ to be placed on the right of $\left\langle z_{k}\right\rangle^{\prime}$. Therefore, the induced $(k-1)$-FAT obstruction is forced.

(c) The global zig-zag pattern forced by a $k$-FAT obstruction, with $k$ nested levels going across $\left\langle y_{k}\right\rangle^{\prime}$ and $\left\langle z_{k}\right\rangle^{\prime}$. It is an obstruction since $P_{1}$ going from $x_{1}$ to $z_{1}$ with all inner vertices non-adjacent to $y_{1}$ cannot be placed. 
with shared endpoints in $\mathcal{R}^{\prime}$; see Fig. 5. We have two pre-drawn vertices $u$ and $v$ such that $r(u)=\ell(v)$ (possibly $u=v$, so only one interval may be pre-drawn). Further, there are two non-adjacent vertices $x$ and $y$, both adjacent to $u$ and $v$. These representations cannot be extended because $\langle x\rangle$ and $\langle y\rangle$ would need to pass through $r(u)=\ell(v)$, but in this case $x$ and $y$ would be adjacent.

If $u \neq v$, the minimality requires that $\ell(u)<\ell(v)=r(u)<r(v)$.

$\boldsymbol{k}$-FAT obstructions. The class of forced asteroidal triple obstructions is defined inductively; the first two obstructions 1-FAT and 2-FAT are depicted in Fig. 3a and b, respectively.

A 1-FAT obstruction consists of three pre-drawn non-adjacent vertices $x_{1}, y_{1}$ and $z_{1}$ such that $\left\langle y_{1}\right\rangle^{\prime}$ is between $\left\langle x_{1}\right\rangle^{\prime}$ and $\left\langle z_{1}\right\rangle^{\prime}$. Further, $x_{1}$ and $z_{1}$ are connected by an induced path $P_{1}$ and $y_{1}$ is non-adjacent to the inner vertices of $P_{1}$. See Fig. 3a. These representations cannot be extended because, for at least one of the inner vertices $u$ of $P_{1}$, $\langle u\rangle$ would intersect $\left\langle y_{1}\right\rangle^{\prime}$, but then $u$ and $y_{1}$ would be adjacent.

A $k$-FAT obstruction (for $k>1$ ) is defined as follows; see Fig. 6a. Let $H_{k-1}$ be the graph of a $(k-1)$-FAT obstruction. To get $H_{k}$, we add to $H_{k-1}$ two vertices $x_{k}$ and $t_{k}$ connected by an induced path $P_{k}$. Concerning edges, $t_{k}$ is adjacent to all vertices of $H_{k-1}$, except for $x_{k-1}$. All vertices of $H_{k-1}$ are non-adjacent to $x_{k}$ and to the inner vertices of $P_{k}$. We put $y_{k}=z_{k-1}$ and $z_{k}=y_{k-1}$. A $k$-FAT obstruction has three pre-drawn vertices $x_{k}, y_{k}$ and $z_{k}$ such that $\left\langle y_{k}\right\rangle^{\prime}$ is between $\left\langle x_{k}\right\rangle^{\prime}$ and $\left\langle z_{k}\right\rangle^{\prime}$. Finally, for $k>1$, we allow $P_{1}$ to be a single edge, so $x_{1}$ can be adjacent to $z_{1}$.

We next see that these representations cannot be extended. Without loss of generality, we assume that $\left\langle x_{k}\right\rangle^{\prime}$ is to the left of $\left\langle y_{k}\right\rangle^{\prime}$, which in turn is to the left of $\left\langle z_{k}\right\rangle^{\prime}$; see Fig. 6b. Since the inner vertices of $P_{k}$ are not adjacent to any vertex of $H_{k-1}, \ell\left(t_{k}\right)<\ell\left(y_{k}\right)$ and $r\left(t_{k}\right)>\ell\left(z_{k}\right)$. Since $x_{k-1}$ is not adjacent to $t_{k},\left\langle x_{k-1}\right\rangle$ is either to the right or to the left of $\left\langle t_{k}\right\rangle$. If it was to its left, then it would be to the left of $\left\langle x_{k}\right\rangle^{\prime}$. Since the inner vertices of $P_{k}$ are not adjacent to any vertex of $H_{k-1}$, we would obtain that $H_{k-1}$ is disconnected, reaching a contradiction. Therefore, $\left\langle x_{k-1}\right\rangle$ is to the right of $\left\langle t_{k}\right\rangle$ and, in consequence, to the right of $\left\langle z_{k}\right\rangle^{\prime}$. In general, $\left\langle x_{k-1}\right\rangle$ is forced to be placed on the other side of $\left\langle z_{k}\right\rangle^{\prime}=\left\langle y_{k-1}\right\rangle^{\prime}$ than $\left\langle y_{k}\right\rangle^{\prime}=\left\langle z_{k-1}\right\rangle^{\prime}$. Then, the $(k-1)$-FAT obstruction of $H_{k-1}$ is forced. Since 1-FAT is a obstruction, we obtain that $k$-FAT, for $k>1$, is also an obstruction. The global structure forced by a $k$-FAT obstruction is depicted in Fig. 6c. The main structural lemma of this paper, $k$-FAT Lemma 22, explains in which situations these complicated $k$-FAT obstructions occur.

The remaining nine classes, depicted in Fig. 7, are derived from $k$-FAT obstructions. Let $H_{k}$ denote the graph of a $k$-FAT obstruction. Except for the class of $(k, \ell)$-CE obstructions, we create the graphs $\widetilde{H}_{k}$ of the remaining obstructions by adding a few vertices to $H_{k}$. These vertices are pre-drawn, and their positions force $x_{k}, y_{k}$ and $z_{k}$ to be represented as in their pre-drawn positions in a $k$-FAT obstruction. Since partial representations of $k$-FAT obstructions cannot be extended, partial representations of these derived classes cannot be extended either.

For $k=1$, when one of $x_{1}$ and $z_{1}$ is not pre-drawn, we also allow $x_{1}$ to be adjacent to $z_{1}$. Additionally, it is possible that the added vertices already belong to $H_{k}$; for instance, 


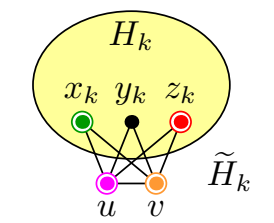

(a)
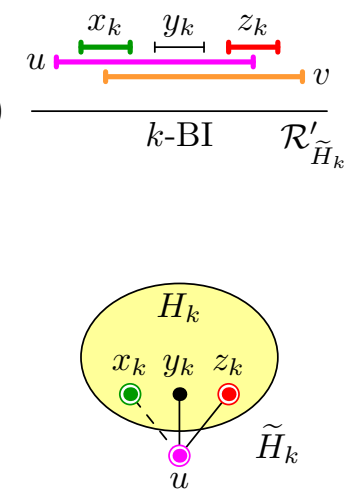

(d)

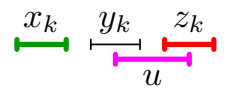

$k$-FB $\mathcal{R}_{\widetilde{H}_{k}}^{\prime}$

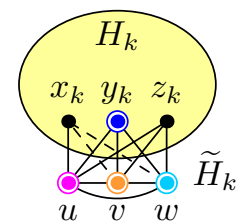

(g)

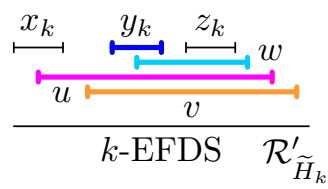

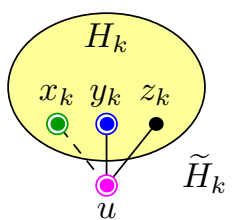

(b)
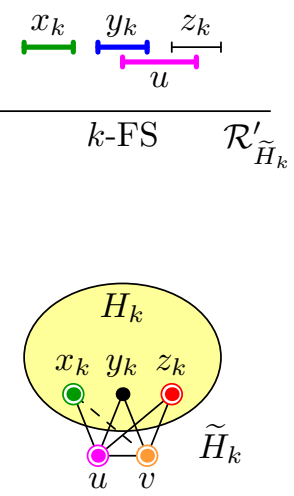

(e)
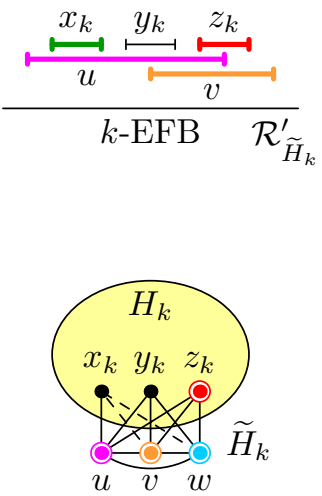

(h)

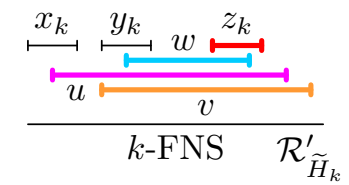

(c)
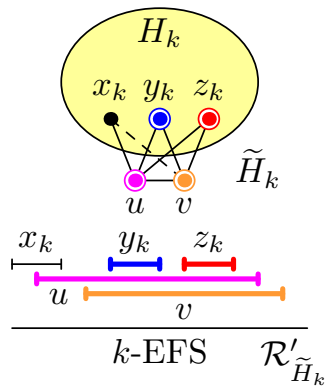

(f)
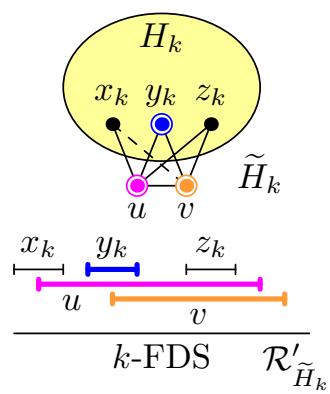

(i)
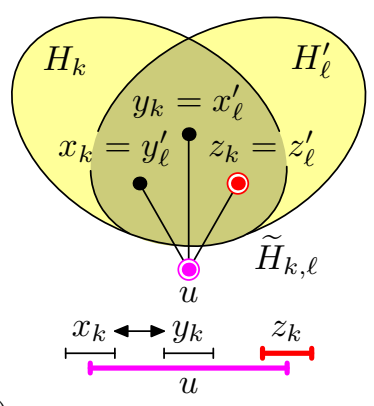

$$
(k, \ell)-\mathrm{CE} \mathcal{R}_{\widetilde{H}_{k, \ell}^{\prime}}^{\prime}
$$

Figure 7: Nine classes of obstructions derived from $k$-FAT obstructions.

a $k$-BI obstruction may have $u=t_{k}$ or $v=t_{k}$. Also, we do not specify in details the edges between the added vertices and $H_{k} \backslash\left\{x_{k}, y_{k}, z_{k}\right\}$. An accurate description would be too lengthy and the reader may derive it from Fig. 6c. We finally remark that we also consider all of the obstructions from the list horizontally flipped.

$\boldsymbol{k}$-BI obstructions. The class of blocked intersection obstructions is shown in Fig. 7a. To create $\widetilde{H}_{k}$ from $H_{k}$, we add two vertices $u$ and $v$ adjacent to $x_{k}, y_{k}$ and $z_{k}$. Then the partial representation contains four pre-drawn vertices $x_{k}, z_{k}, u$ and $v$. We have $\ell(u) \leqslant \ell(v)<r(u) \leqslant r(v),\left\langle x_{k}\right\rangle^{\prime}$ covering $\ell(v)$, and $\left\langle z_{k}\right\rangle^{\prime}$ covering $r(u)$. We allow $u=v$.

The minimality further implies that $k \leqslant 2$. The reason is that a $k$-BI obstruction with $k>3$ contains a smaller $(k, 1)$-CE obstruction by removing $v$ and freeing $\left\langle x_{k}\right\rangle^{\prime}$ (this follows from Lemma 23).

Concerning 1-BI, we allow $x_{1}=z_{1}$. We illustrate all distinct obstructions only for this 
(a)
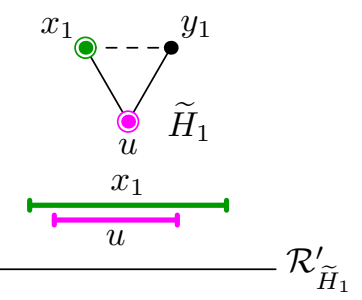

$\ell\left(x_{1}\right) \leq \ell(u)<r(u) \leq r\left(x_{1}\right)$

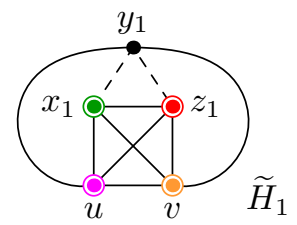

(d)

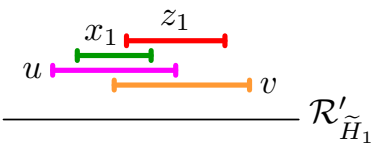

$\ell(u)<\ell\left(x_{1}\right) \leq \ell(v)<\ell\left(z_{1}\right) \leq$ $r\left(x_{1}\right)<r(u) \leq r\left(z_{1}\right)<r(v)$ (b)
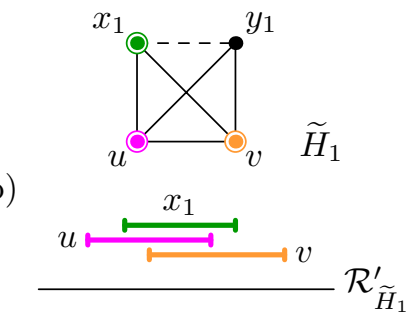

$\ell(u)<\ell\left(x_{1}\right) \leq \ell(v)<$ $r(u) \leq r\left(x_{1}\right)<r(v)$

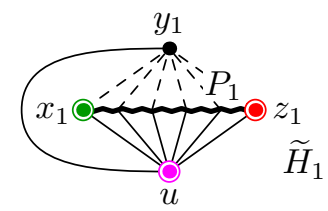

(e)

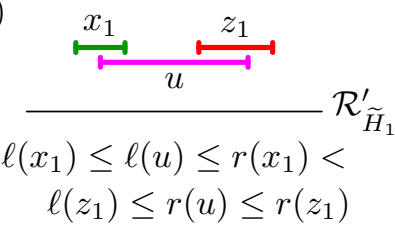

(c)
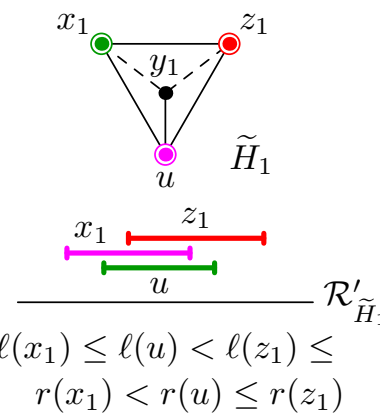

(f)
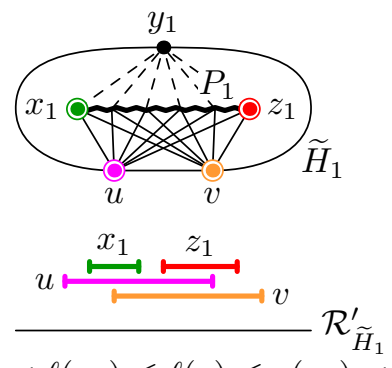

$\ell(u)<\ell\left(x_{1}\right) \leq \ell(v) \leq r\left(x_{1}\right)<$

$\ell\left(z_{1}\right) \leq r(u) \leq r\left(z_{1}\right)<r(v)$

Figure 8: All minimal 1-BI obstructions are depicted. Each gives several 1-BI obstructions with different $\mathcal{R}_{\widetilde{H}_{1}}^{\prime}$, since there are several possible orderings of the endpoints satisfying the given inequalities. We have $x_{1}=z_{1}$ for (a) and (b), and $x_{1} \neq z_{1}$ otherwise. We have $u=v$ for (a), (c) and (e), and $u \neq v$ otherwise.

particular case $(k=1)$, so that the reader can understand the complexity of these classes; see Fig. 8.

For $k=2$, we know by Lemma 23 that $x_{2}$ is adjacent to $t_{2}$. The pre-drawn intervals are as follows:

$$
\begin{array}{rlrl}
\ell\left(x_{2}\right) & \leqslant \ell(u) \leqslant r\left(x_{2}\right)<\ell\left(z_{2}\right) \leqslant r(u) \leqslant r\left(z_{2}\right), & & \text { for } u=v, \\
\ell(u)<\ell\left(x_{2}\right) \leqslant \ell(v) \leqslant r\left(x_{2}\right)<\ell\left(z_{2}\right) \leqslant r(u) \leqslant r\left(z_{2}\right)<r(v), & & \text { for } u \neq v .
\end{array}
$$

The position of $\langle u\rangle^{\prime}$ and $\langle v\rangle^{\prime}$ forces $\left\langle y_{2}\right\rangle$ to be placed between $\left\langle x_{2}\right\rangle^{\prime}$ and $\left\langle y_{2}\right\rangle^{\prime}$ in every extending representation, which forces a 2-FAT obstruction.

$\boldsymbol{k}$-FS obstructions. The class of forced side obstructions is shown in Fig. 7b. To create $\widetilde{H}_{k}$ from $H_{k}$, we add a vertex $u$ adjacent to $y_{k}$ and $z_{k}$. The partial representation contains three pre-drawn vertices $x_{k}, y_{k}$ and $u$. We have $\ell\left(y_{k}\right) \leqslant \ell(u) \leqslant r\left(y_{k}\right)<r(u)$, and $\left\langle x_{k}\right\rangle^{\prime}$ is on the left of $\left\langle y_{k}\right\rangle^{\prime}$.

$\boldsymbol{k}$-EFS obstructions. The class of extended forced side obstructions is similar to that of $k$-FS obstructions; see Fig. 7 c. To create $\widetilde{H}_{k}$ from $H_{k}$, we add $u$ adjacent to $x_{k}, y_{k}$, and $z_{k}$, and $v$ adjacent to $y_{k}$ and $z_{k}$. The partial representation contains four vertices $y_{k}, z_{k}$, $u$ and $v$ pre-drawn as follows:

$$
\ell(u)<\ell(v)<\ell\left(y_{k}\right) \leqslant r\left(y_{k}\right)<\ell\left(z_{k}\right) \leqslant r\left(z_{k}\right)<r(u) \leqslant r(v) .
$$


$\boldsymbol{k}$-FB obstructions. The class of forced betweenness obstructions is similar to $k$-BI obstructions with $u=v$; see Fig. $7 \mathrm{~d}$. To create $\widetilde{H}_{k}$ from $H_{k}$, we add $u$ adjacent to $y_{k}$ and $z_{k}$. The partial representation contains three pre-drawn vertices $x_{k}, z_{k}$, and $u$. We have $\ell(u)<\ell\left(z_{k}\right) \leqslant r(u) \leqslant r\left(z_{k}\right)$, and $\left\langle x_{k}\right\rangle^{\prime}$ is pre-drawn on the left of $\langle u\rangle^{\prime}$.

$\boldsymbol{k}$-EFB obstructions. The class of extended forced betweenness obstructions is similar to those of $k$-BI and $k$-FB obstructions; see Fig. 7 e. To create $\widetilde{H}_{k}$ from $H_{k}$, we add $u$ adjacent to $x_{k}, y_{k}$ and $z_{k}$, and $v$ adjacent to $y_{k}$ and $z_{k}$. The partial representation contains four pre-drawn vertices $x_{k}, z_{k}, u$, and $v$. We have

$$
\ell(u)<\ell\left(x_{k}\right) \leqslant r\left(x_{k}\right)<\ell(v)<\ell\left(z_{k}\right) \leqslant r(u) \leqslant r\left(z_{k}\right)<r(v) .
$$

$\boldsymbol{k}$-FDS obstructions. The class of forced different sides obstructions is shown in Fig. $7 \mathrm{f}$. To create $\widetilde{H}_{k}$ from $H_{k}$, we add $u$ adjacent to $x_{k}, y_{k}$, and $z_{k}$, and $v$ adjacent to $y_{k}$ and $z_{k}$. The partial representation contains three vertices $y_{k}, u$ and $v$ pre-drawn as follows:

$$
\ell(u)<\ell\left(y_{k}\right) \leqslant \ell(v) \leqslant r\left(y_{k}\right)<r(u) \leqslant r(v) .
$$

$\boldsymbol{k}$-EFDS obstructions. The class of extended forced different sides obstructions is similar to that of $k$-FDS obstructions; see Fig. $7 \mathrm{~g}$. To the construction of $k$-FDS, we further add $w$ adjacent to $y_{k}$ and $z_{k}$. The partial representation contains four vertices $y_{k}, u, v$ and $w$ pre-drawn as follows:

$$
\ell(u)<\ell(v)<\ell\left(y_{k}\right) \leqslant \ell(w) \leqslant r\left(y_{k}\right)<r(w)<r(u) \leqslant r(v) .
$$

$\boldsymbol{k}$-FNS obstructions. The class of forced nested side obstructions is constructed similarly to that of $k$-EFDS obstructions. In this case, $z_{k}$ is pre-drawn instead of $y_{k}$; see Fig. 7h. In $\mathcal{R}_{\widetilde{H}_{k}}^{\prime}$, we have

$$
\ell(u)<\{\ell(v), \ell(w)\} \leqslant \ell\left(z_{k}\right) \leqslant r(w)<r(u) \leqslant r(v),
$$

where $\ell(v)$ and $\ell(w)$ are ordered arbitrarily.

$(\boldsymbol{k}, \ell)-\mathrm{CE}$ obstructions. The class of covered endpoint obstructions is created from a $k$-FAT obstruction glued to an $\ell$-FAT obstruction; see Fig. 7 i. To create $\widetilde{H}_{k, \ell}$, we glue $H_{k}$ to $H_{\ell}^{\prime}$, with $k \geqslant \ell$. We put $z_{k}=z_{\ell}^{\prime}, x_{k}=y_{\ell}^{\prime}$ and $y_{k}=x_{\ell}^{\prime}$, and some other vertices of these obstructions may also be shared. We add $u$ adjacent to $x_{k}, y_{k}$, and $z_{k}$. The partial representation contains two pre-drawn intervals $\left\langle z_{k}\right\rangle^{\prime}$ and $\langle u\rangle^{\prime}$ such that $\ell(u)<\ell\left(z_{k}\right) \leqslant r(u) \leqslant r\left(z_{k}\right)$. These representations cannot be extended because, if $\left\langle x_{k}\right\rangle$ is placed to the left of $\left\langle y_{k}\right\rangle$, we get a $k$-FAT obstruction, while if $\left\langle x_{k}\right\rangle$ is placed to the right of $\left\langle y_{k}\right\rangle$, we get an $\ell$-FAT obstruction.

By Lemma 23, for $k>2$, if we swap the positions of $\left\langle x_{k}\right\rangle^{\prime}$ and $\left\langle y_{k}\right\rangle^{\prime}$ in a $k$-FAT obstruction, we obtain a 1-FAT obstruction. For $k>2$, this implies that a minimal $(k, \ell)$-CE obstruction is a $(k, 1)$-CE obstruction and it is formed by the graph $H_{k}$ of a $k$-FAT obstruction together with an added vertex $u$. (By a careful analysis, either $u$ is adjacent to all vertices of $H_{k}$, or $u=t_{k}$.) Hence, the only $(k, \ell)$-CE obstructions that are minimal are $(k, 1)$-CE obstructions and $(2,2)$-CE obstructions; in other words, either $\ell=1$, or $k=\ell=2$. The remaining $(k, \ell)$-CE obstructions, where $2 \geqslant k \geqslant \ell$, are analysed in Lemma 25 and depicted in Fig. 9. 
$(1,1)-\mathrm{CE}$

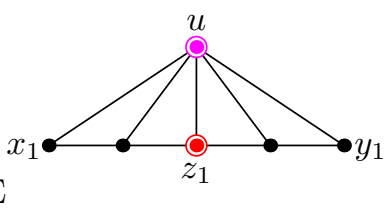

(b)

$(2,1)-\mathrm{CE}$

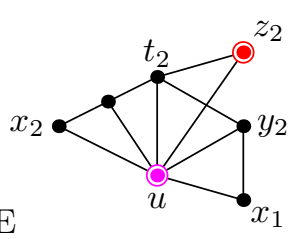

(c)

$(2,2)-\mathrm{CE}$

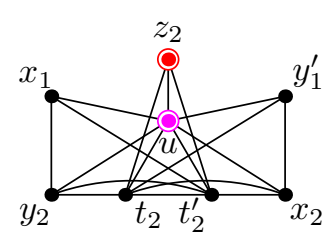

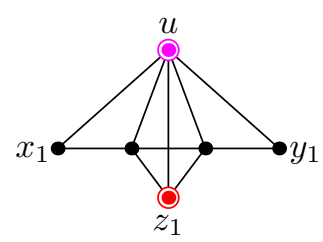
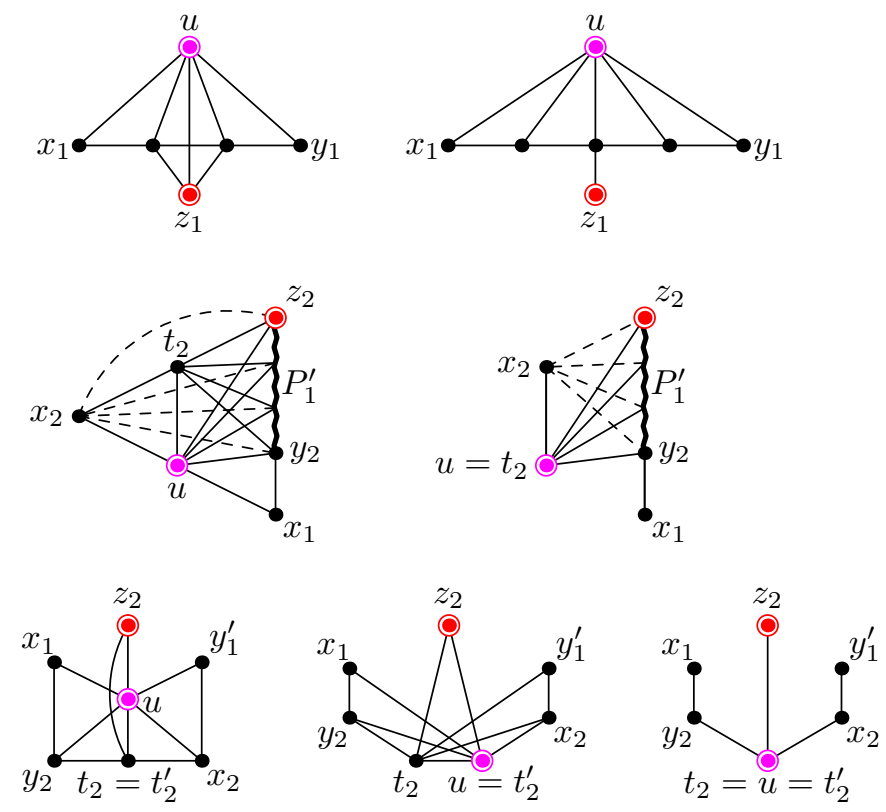

Figure 9: (a) There are three minimal $(1,1)$-CE obstructions, and they are all finite graphs. The reason is that if, say, a path $P_{1}$ was very long, we could replace $x_{1}$ by an inner vertex of $P_{1}$; so such an obstruction would not be minimal.

(b) There are three minimal $(2,1)$-CE obstructions. Due to the minimality, $P_{2}$ is a path of length at most two. When $x_{2}$ and $t_{2}$ are non-adjacent, then $P_{1}^{\prime}=y_{2} t_{2} z_{2}$ is a path avoiding $N\left[x_{2}\right]$. When $x_{2}$ and $t_{2}$ are adjacent, there are two cases, namely, $u \neq t_{2}$, and $u=t_{2}$. The path $P_{1}^{\prime}$ is of length at least two.

(c) There are four minimal $(2,2)$-CE obstructions, and they are all finite graphs. Necessarily $x_{2} t_{2}$ and $y_{2} t_{2}^{\prime}$ are edges, and we can choose $x_{2}$ in such a way that it is adjacent to $y_{1}^{\prime}$ (similarly, $y_{2}$ is adjacent to $x_{1}$ ). There are four different graphs, because the vertices $u, t_{2}$ and $t_{2}^{\prime}$ might be distinct or not.

\subsection{Proofs of Non-extendibility and Minimality}

We sketch proofs that the defined obstructions are non-extendible and minimal. This implies the first part of Theorem 1. We establish the harder implication in Sections 5, 6, 7,8 , and 9 .

Lemma 4. Every k-FAT obstruction is non-extendible and minimal.

Proof. We prove the claim by induction. For $k=1$, non-extendibility and minimality are clear. For $k>1$, assume that $\left\langle x_{k}\right\rangle^{\prime}$ is on the left of $\left\langle y_{k}\right\rangle^{\prime}$, and $\left\langle y_{k}\right\rangle^{\prime}$ is on the left of $\left\langle z_{k}\right\rangle^{\prime}$. In every representation of $k$-FAT, $\left\langle t_{k}\right\rangle$ covers $\left[\ell\left(y_{k}\right), \ell\left(z_{k}\right)\right]$. We know that $\left\langle x_{k-1}\right\rangle$ cannot be on the left of $\left\langle x_{k}\right\rangle^{\prime}$, since $H_{k-1}$ is connected and $x_{k}$ is non-adjacent to all vertices of $H_{k-1}$. Therefore $\left\langle x_{k-1}\right\rangle$ has to be placed on the right of $\left\langle z_{k}\right\rangle^{\prime}$. We get a $(k-1)$-FAT obstruction, which is non-extendible by the induction hypothesis.

It remains to argue the minimality. If one of $\left\langle x_{k}\right\rangle^{\prime},\left\langle y_{k}\right\rangle^{\prime}$ and $\left\langle z_{k}\right\rangle^{\prime}$ is made free, we 
can place them in such a way that $\left\langle z_{k}\right\rangle$ is between $\left\langle x_{k}\right\rangle$ and $\left\langle y_{k}\right\rangle$. This makes the partial representation extendible: It works for $k=1$, and for $k>1$, we can place $x_{k-1}$ on the right of $\left\langle y_{k}\right\rangle$, which makes the induced $H_{k-1}$ extendible. If we remove one of the vertices or induced paths, the argument is similar.

Lemma 5. The following obstructions are non-extendible and minimal:

- $S E, k-F S, k$-EFS, $k-F B, k-E F B, k-F D S, k$-EFDS, and $k$-FNS obstructions,

- $k$-BI obstructions for $k \leqslant 2$,

- $(k, \ell)-C E$ obstructions where either $\ell=1$, or $k=\ell=2$.

Proof. For SE obstructions, the proof is trivial. For the remaining classes (aside $k$-BI and $(k, \ell)-\mathrm{CE})$, we proceed as follows. Non-extendibility follows from the fact that, in all cases, $\left\langle y_{k}\right\rangle$ is forced to be placed between $\left\langle x_{k}\right\rangle$ and $\left\langle z_{k}\right\rangle$. To show minimality, we use the minimality of $k$-FAT obstructions. Then it is easy to show that freeing any added pre-drawn interval or removing any added vertex results in the possibility of placing $\left\langle z_{k}\right\rangle$ between $\left\langle x_{k}\right\rangle$ and $\left\langle y_{k}\right\rangle$.

Consider a $k$-BI where $k \leqslant 2$. Non-extendibility follows from the fact that $\left\langle y_{k}\right\rangle$ has to be placed between $\left\langle x_{k}\right\rangle^{\prime}$ and $\left\langle z_{k}\right\rangle^{\prime}$, thus forcing the k-FAT obstruction, which is nonextendible by Lemma 4. By removing a vertex or an induced path of $H_{k}$, it becomes extendible as argued in Lemma 4. By freeing $\langle u\rangle^{\prime}$ or $\left\langle z_{k}\right\rangle^{\prime}$, we can place $\left\langle y_{k}\right\rangle$ on the right of $\left\langle z_{k}\right\rangle^{\prime}$ which makes the partial representation extendible. By freeing $\langle v\rangle^{\prime}$ or $\left\langle x_{k}\right\rangle^{\prime}$, we can place $\left\langle y_{k}\right\rangle$ on the left of $\left\langle x_{k}\right\rangle^{\prime}$, which also makes it extendible because $k \leqslant 2$ and $x_{2}$ is adjacent to $t_{2}$.

For $\left(k, \ell\right.$ )-CE, either $\left\langle y_{k}\right\rangle$ is between $\left\langle x_{k}\right\rangle$ and $\left\langle z_{k}\right\rangle^{\prime}$ (non-extendible due to the $k$-FAT obstruction), or $\left\langle y_{\ell}\right\rangle$ is between $\left\langle x_{\ell}\right\rangle$ and $\left\langle z_{\ell}\right\rangle^{\prime}$ (non-extendible due to the $\ell$-FAT obstruction). Minimality is also easy: Removing or freeing $u$ allows to place $\left\langle z_{k}\right\rangle^{\prime}$ between $\left\langle x_{k}\right\rangle$ and $\left\langle y_{k}\right\rangle$, which is extendible. And removing anything from one of the FAT obstructions allows one of the orderings of $\left\langle x_{k}\right\rangle$ and $\left\langle y_{k}\right\rangle$ to be extendible.

We note that the list of minimal obstructions is unique. Indeed, every minimal obstruction itself corresponds to a valid input, which cannot be obstructed by a distinct obstruction due to the minimality. Therefore, it is not possible to construct a smaller list of minimal obstructions, or to argue that if the partial representation contains a particular obstruction, then it also contains an additional one.

\section{Maximal Cliques and MPQ-trees}

In this section, we review well-known properties of interval graphs. First, we describe their characterization in terms of orderings of maximal cliques. Then, we introduce two data structures to deal with these orderings, namely, PQ-trees and MPQ-trees. Finally, we prove some simple structural results concerning MPQ-trees.

Consecutive Orderings. Fulkerson and Gross [14] proved the following fundamental characterization of interval graphs: 
Lemma 6 (Fulkerson and Gross [14]). A graph is an interval graph if and only if there exists a linear ordering < of its maximal cliques such that, for each vertex, the maximal cliques containing this vertex appear consecutively.

We call an ordering of the maximal cliques satisfying the statement of Lemma 6 a consecutive ordering. See Fig. 10 for an example. The ordering $<$ from the statement is obtained by sweeping an interval representation from left to right. By the Helly property, the intervals of every maximal clique have a non-empty intersection. For all maximal cliques, these intersections are disjoint and ordered from left to right. In the intersection of the intervals of a maximal clique $a$, we pick one point which we call a clique-point $\operatorname{cp}(a)$. The left-to-right ordering of these clique-points gives $<$. On the other hand, given a consecutive ordering $<$, we place the clique-points from left to right according to $<$ and construct an interval representation by placing each interval on top of its clique-points and no others. This can be done because the ordering places the maximal cliques containing each of the vertices consecutively.

PQ-trees. Booth and Lueker [6] designed a data structure called PQ-trees to efficiently work with consecutive orderings of maximal cliques. A $P Q$-tree $T$ is a rooted tree. Its leaves are in one-to-one correspondence with the maximal cliques. Its inner nodes are of two types: P-nodes and Q-nodes. Each P-node has at least two children, and each Q-node
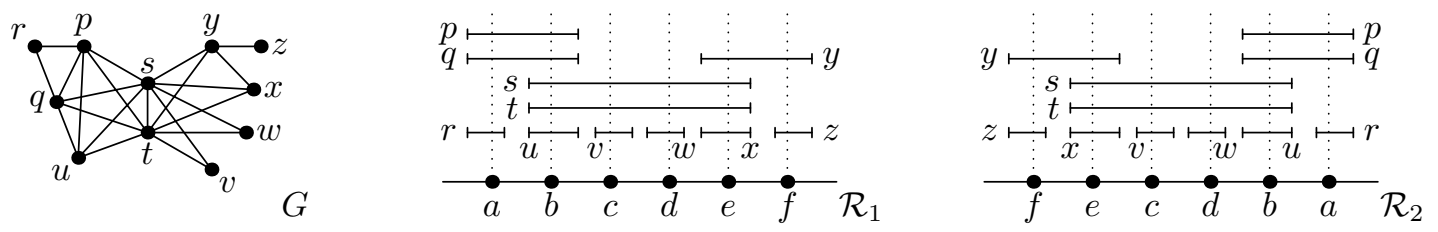

Figure 10: An interval graph $G$ and two of its representations with different left-to-right orderings $<$ of the maximal cliques. Some choices of clique-points are depicted on the real lines.

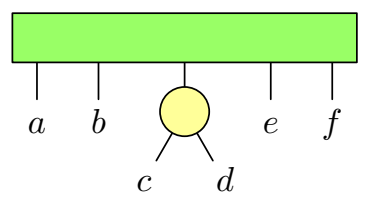

(a)
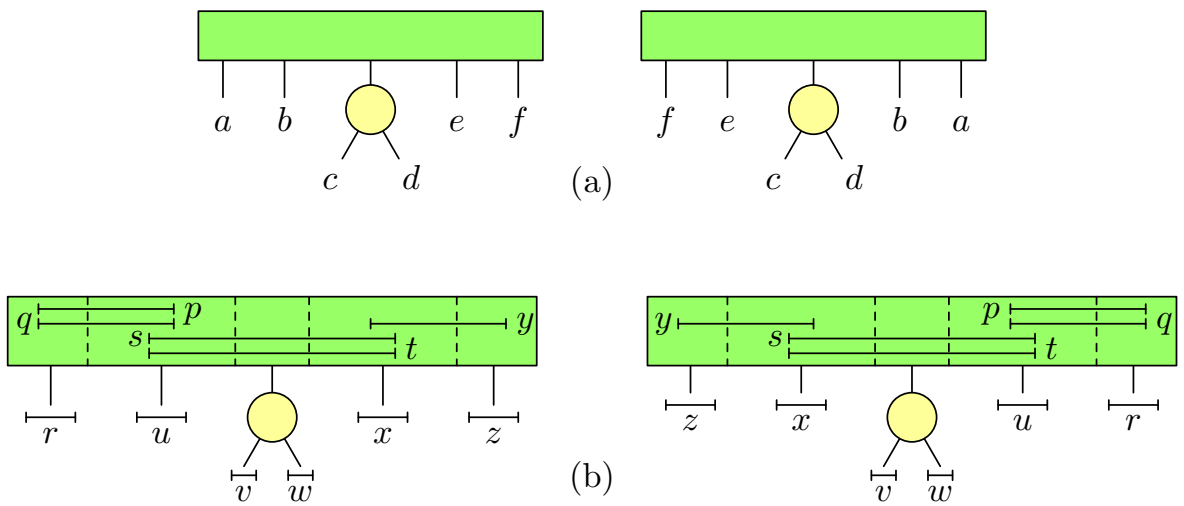

Figure 11: (a) Two equivalent PQ-trees with frontiers $a<b<c<d<e<f$ and $f<e<c<d<b<a$, respectively. In all figures, we denote P-nodes by circles and Q-nodes by rectangles. (b) The corresponding MPQ-trees with depicted sections. 
has at least three. Further, for every inner node, a left-to-right ordering of its children is given. Every PQ-tree $T$ represents one linear ordering $<_{T}$ of the maximal cliques called the frontier of $T$, which is the ordering of the leaves from left to right.

Every PQ-tree $T$ further represents other linear orderings. These orderings are frontiers of equivalent PQ-trees. A PQ-tree $T^{\prime}$ is equivalent to $T$ if it can be constructed from $T$ by a sequence of equivalent transformations of two types: (i) an arbitrary reordering of the children of a P-node, and (ii) a reversal of the order of the children of a Q-node. Fig. 11a depicts two equivalent PQ-trees corresponding to the interval graph from Fig. 10.

Booth and Lueker proved that, for every interval graph, there exists a unique PQ-tree (up to equivalence transformations) representing precisely the consecutive orderings of the maximal cliques. In other words, this tree describes all possible interval representations of this interval graph.

A subtree of $T$ consists of a node and all its descendants. The subtrees of a node $N$ are those subtrees having the children of $N$ as the roots. For a node $N$, let $T[N]$ denote the subtree of $T$ with the root $N$.

MPQ-trees. For the purpose of this paper, we need more information about the way in which the vertices of the interval graph are related to the structure of the PQ-tree. This additional information is contained in the modified PQ-tree (MPQ-tree), introduced by Korte and Möhring [28]. We note that the same idea is already present in the earlier paper of Colbourn and Booth [10].

The MPQ-tree is an augmentation of the PQ-tree in which the nodes of $T$ have assigned subsets of $V(G)$ called sections. To a leaf representing a clique $a$, we assign one section $s(a)$. Similarly, to each P-node $P$, we assign one section $s(P)$. For a Q-node $Q$ with $n$ children, we have $n$ sections $s_{1}(Q), \ldots, s_{n}(Q)$, each corresponding to one subtree of $Q$.

The section $s(a)$ has all vertices contained in the maximal clique $a$ and no other maximal clique. The section $s(P)$ of a $\mathrm{P}$-node $P$ has all vertices that are contained in all maximal cliques of $T[P]$ and in no other maximal clique. Sections of Q-nodes are more complicated. Let $Q$ be a $Q$-node with subtrees $T_{1}, \ldots, T_{n}$. Let $x$ be a vertex contained only in maximal cliques of $T[Q]$, and suppose that it is contained in maximal cliques of at least two subtrees. Then $x$ is contained in every section $s_{i}(Q)$ such that some maximal clique of $T_{i}$ contains $x$. Fig. 11b depicts the sections for the example in Fig. 10.

Korte and Möhring [28] state the following properties:

- Every vertex $x$ is placed in the sections of exactly one node of $T$. In the case of a Q-node, it is placed in consecutive sections of this node.

- For a Q-node $Q$, if $x$ is placed in a section $s_{i}(Q)$, then $x$ is contained in all cliques of $T_{i}$.

- Every section of a Q-node is non-empty. Moreover, two consecutive sections have a non-empty intersection.

- A maximal clique contains exactly those vertices contained in the sections encountered when we traverse the tree from the corresponding leaf to the root. 
Structure of MPQ-trees. Next, we show several structural properties used in building minimal obstructions which are quite easy to prove:

Lemma 7. Let $Q$ be a $Q$-node. Then $s_{i}(Q) \neq s_{j}(Q)$ for every $i \neq j$. Further, if $s_{i}(Q) \subsetneq$ $s_{i+1}(Q)$, then at least one section of $T_{i}$ is non-empty.

Proof. If $s_{i}(Q)=s_{j}(Q)$, then we could exchange $T_{i}$ and $T_{j}$ and we would obtain a valid MPQ-tree for $G$. Since $n \geqslant 3$, this yields a contradiction with the fact that the only possible transformation of a $Q$-node is reverting the order of its children.

For the latter part, let $a$ and $b$ respectively be maximal cliques of a leaf in $T_{i}$ and a leaf in $T_{i+1}$. Then $a \backslash b \neq \emptyset$ and every $x \in a \backslash b$ belongs to sections of $T_{i}$.

Let $N$ be a node of the MPQ-tree. By $G[N]$ we denote the subgraph induced by all the vertices in the sections of the subtree rooted at $N$. Similarly, for a subtree $T^{\prime}$, we denote the subgraph induced by the vertices in its sections by $G\left[T^{\prime}\right]$.

Lemma 8. Let $N$ be an inner node of an $M P Q$-tree.

(i) If $N$ is a $Q$-node, then $G[N]$ is connected.

(ii) If $N$ is a P-node, then $G[N]$ is connected if and only if $s(N)$ is non-empty. Furthermore, for every child $T_{i}$ of $N$, the graph $G\left[T_{i}\right]$ is connected.

Proof. (i) It follows from the facts that the vertices in any section form a clique, and that any two consecutive sections of $N$ have non-empty intersection.

(ii) The first statement is clear. For the second part, notice that if $G\left[T_{i}\right]$ was not connected, we could permute the connected components of $G\left[T_{i}\right]$ arbitrarily with the other children of $N$. Therefore $T_{i}$ would not be a child of $N$, but $N$ would have one child per each connected component of $G\left[T_{i}\right]$.

Let $Q$ be a Q-node and $i<j$. Let $x$ and $y$ be two vertices of $G[Q]$, where $x$ is either in $T_{i}$, or $s_{i}(Q)$, and $y$ is either in $T_{j}$, or $s_{j}(Q)$. A path $P_{x, y}$ is called $Q$-monotone if all inner vertices of the path belong to the sections of $Q$, their leftmost sections strictly increase, and their rightmost ones strictly increase as well.

Lemma 9. Let $H$ be an induced subgraph of $G[Q]$ such that $x, y \in V(H)$ belong to one component. Then every shortest path $P_{x, y}$ in $H$ is $Q$-monotone.

Proof. It is easy to see that any path from $x$ to $y$ that is not $Q$-monotone can be shortened.

Let $Q$ be a Q-node. Let $u$ be a vertex appearing in sections of $T[Q]$. If $u$ belongs to sections of $Q$, let $s_{u}^{\leftarrow}(Q)$ be the leftmost section of $Q$ containing $u$ and $s_{u}(Q)$ be the rightmost one. If $u$ belongs to sections of a subtree $T_{i}$ of $Q$, we put $s_{u}^{\leftarrow}(Q)=s_{u}(Q)=$ $s_{i}(Q)$. If $s_{u}(Q)$ is on the left of $s_{v}^{\leftarrow}(Q)$, then we say that $u$ is on the left of $v$ and $v$ is on the right of $u$. Also, $u$ and $v$ are on the same side of $w$ if they are both on the left of $w$, or both on the right of $w$. Similarly, $v$ is between $u$ and $w$ if either $u$ is on the left 
and $w$ is on the right of $v$, or $u$ is on the right and $w$ is on the left of $v$. For a maximal clique $a \in T_{i}$, we say that $u$ is on the left of $a$ when $s_{u}(Q)$ is on the left of $s_{i}(Q)$; the other relations are defined similarly.

Non-adjacencies of Maximal Cliques. Maximal cliques of interval graphs have the following special property, which we use to build minimal obstructions.

Lemma 10. Let $H$ be a connected subgraph of an interval graph and let $c$ be a maximal clique with no vertex in $V(H)$. There exists $x \in c$ non-adjacent to all vertices of $V(H)$.

Proof. Consider an interval representation $\mathcal{R}$. It places all intervals of $H$ to one side of $\operatorname{cp}(c)$, say on the left. Let $x$ be the interval of $c$ having the rightmost left endpoint. If $x$ is adjacent to some vertex $y \in V(H)$, then every vertex of $c$ is adjacent to $y$. Since $c$ is maximal, it follows that $y \in c$, contradicting the assumption. So $x$ is non-adjacent to all vertices of $V(H)$.

\section{Characterizing Extendible Partial Representations by Maxi- mal Cliques}

In this section, we explain the characterization of extendible partial representations due to Klavík et al. [23].

Restricting Clique-points. Suppose that there exists a representation $\mathcal{R}$ extending $\mathcal{R}^{\prime}$. Then $\mathcal{R}$ gives some consecutive ordering $<$ of the maximal cliques from left to right. We want to show that the pre-drawn intervals give constraints in the form of a partial ordering $\triangleleft$, defined below. Fig. 12 illustrates examples of constraints posed by a pair of pre-drawn intervals.

For a maximal clique $a$, let $P(a)$ denote the set of all pre-drawn intervals that are contained in $a$. Recall that a clique-point $\operatorname{cp}(a)$ is some point chosen from the intersection of all intervals of $a$ in the representation $\mathcal{R}$. Then $P(a)$ restricts the possible position of the clique-point $\operatorname{cp}(a)$ to only those points $x$ of the real line which are covered in $\mathcal{R}^{\prime}$ by the pre-drawn intervals of $P(a)$ and no others. We denote the set of these admissible

(a)

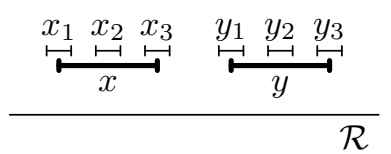

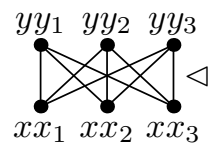

(b)

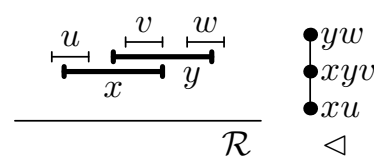

(c)

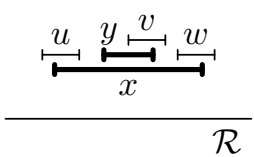

Figure 12: Possible relative positions of pre-drawn intervals $\langle x\rangle^{\prime}$ and $\langle y\rangle^{\prime}$, and some examples of the Hasse diagrams of the posed constraints. (a) All maximal cliques containing $x$ have to be on the left of those containing $y$. (b) All maximal cliques containing $x$ have to be on the left of those containing both $x$ and $y$, which are on the left of those containing only $y$. (c) An inclusion of pre-drawn intervals poses no constraints. A maximal clique containing only $x$ can be either on the left, or on the right of the maximal cliques containing both $x$ and $y$. 
(a)

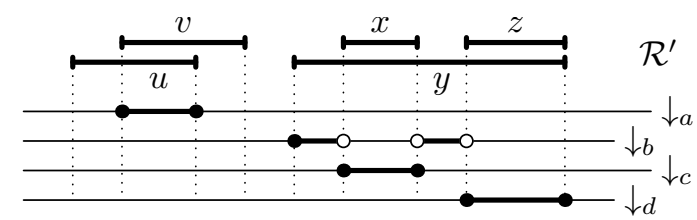

(b)

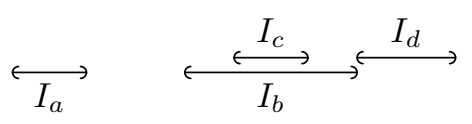

Figure 13: (a) Four maximal cliques $a, b, c$, and $d$ with $P(a)=\{u, v\}, P(b)=\{y\}$, $P(c)=\{x, y\}$, and $P(d)=\{y, z\}$. The possible positions $\downarrow_{a}, \downarrow_{b}, \downarrow_{c}$, and $\downarrow_{d}$ of their clique-points are illustrated. (b) The corresponding open intervals $I_{a}, I_{b}, I_{c}$, and $I_{d}$.

positions by $\downarrow_{a}$. Formally:

$$
\downarrow_{a}=\left\{x: x \in \mathbb{R} \text { and } x \in\langle u\rangle^{\prime} \Longleftrightarrow u \in P(a)\right\} ;
$$

for examples see Fig. 13a. Equivalently, $\downarrow_{a}$ is defined in [2] as

$$
\downarrow_{a}=\left(\bigcap_{u \in P(a)}\langle u\rangle^{\prime}\right) \backslash\left(\bigcup_{v \notin P(a)}\langle v\rangle^{\prime}\right) .
$$

We are interested in the extremal points of $\downarrow_{a}$. By $\curvearrowleft(a)$ (resp. $\curvearrowright(a)$ ), we denote the infimum (resp. the supremum) of $\downarrow_{a}$. We use an open interval $I_{a}=(\curvearrowleft(a), \curvearrowright(a))$ to represent $\downarrow_{a}$. We note that this does not imply that $\downarrow_{a}$ contains all points between $\curvearrowleft(a)$ and $\curvearrowright(a)$; see $\downarrow_{b}$ in Fig. 13. Notice that when $P(a)=\emptyset$, then $I_{a}=\mathbb{R}$.

The Relation $\triangleleft$. For two distinct maximal cliques $a$ and $b$, we write $a \triangleleft b$ if $\curvearrowright(a) \leqslant$ $\curvearrowleft(b)$, or in other words, if $I_{a}$ is on the left of $I_{b}$. We put $a \triangleleft a$ when $\downarrow_{a}=\emptyset$. The definition of $\triangleleft$ is quite natural, since $a \triangleleft b$ implies that every extending representation $\mathcal{R}$ has to place $\operatorname{cp}(a)$ to the left of $\operatorname{cp}(b)$. For instance, in Fig. 13, we get that $a \triangleleft b \triangleleft d$ and $a \triangleleft c \triangleleft d$, but $b$ and $c$ are incomparable.

All maximal cliques $a$ with $\downarrow_{a} \neq \emptyset$ can be represented by open intervals $I_{a}$. This representation describes $\triangleleft$, since $a \triangleleft b$ if and only if $I_{a}$ and $I_{b}$ are disjoint and $I_{a}$ is on the left of $I_{b}$. An ordering is called an interval ordering if it can be represented by closed intervals in the above manner: $a<b$ if and only if the interval of $a$ is on the left of the interval of $b$. Interval orders are studied in the context of time constraints and have many applications; for instance, see [13]. We get the following:

Lemma 11 (Klavík et al. [23]). The relation $\triangleleft$ is an interval ordering if and only if no maximal clique a has $\downarrow_{a}=\emptyset$ and there are no two distinct maximal cliques $a$ and $b$ such that $\downarrow_{a}=\downarrow_{b}=\{x\}$.

Proof. When $\downarrow_{a}=\emptyset$, then $a \triangleleft a$, so $\triangleleft$ is not an ordering. Similarly, when $\downarrow_{a}=\downarrow_{b}=\{x\}$, we have $a \triangleleft b \triangleleft a$. Otherwise, let us define the closure of any interval $(x, y)$ as $[x, y]$, even when $x=y$. Then $\triangleleft$ can be represented by the closure of the intervals $I_{a}$, where we add a small gap between touching pairs of right and left endpoints that belong to distinct intervals. 
We note that the situations in which $\triangleleft$ is not an interval order can be easily dealt with, leading to a 1-BI or an SE obstruction (Lemmas 16, 19, and 21).

Below, we characterize extendible partial representations $\mathcal{R}^{\prime}$ using $\triangleleft$. Since our characterization of minimal obstructions highly depends on this, we also explain the proof from [23].

Lemma 12 (Klavík et al. [23]). A partial representation $\mathcal{R}^{\prime}$ is extendible if and only if there exists a consecutive ordering of the maximal cliques that extends $\triangleleft$.

Proof. It is obvious that the constraints posed by $\triangleleft$ are necessary.

To show the other implication, suppose that we have a consecutive ordering $<$ of the maximal cliques which extends $\triangleleft$. We construct a representation $\mathcal{R}$ extending $\mathcal{R}^{\prime}$ as follows. We place the clique-points according to $<$ from left to right, in the following greedy manner: Suppose that we want to place a clique-point $\operatorname{cp}(a)$. Let $\mathrm{cp}(b)$ be the last placed clique-point. Consider all the points where the clique-point $\operatorname{cp}(a)$ can be placed and that are to the right of the clique-point $\operatorname{cp}(b)$. If there is a single such point, we place $\operatorname{cp}(a)$ there. Otherwise $\curvearrowleft(a)<\curvearrowright(a)$, and we take the infimum of all such points and place the clique-point $\operatorname{cp}(a)$ to its right by an appropriate epsilon, for example the distance of two closest distinct endpoints of pre-drawn intervals divided by $n$.

We prove by contradiction that this greedy procedure cannot fail; see Fig. 14. Let $\operatorname{cp}(a)$ be the clique-point for which the procedure fails. It is not possible that $\downarrow_{a}=\emptyset$, since in this case $a \triangleleft a$ and $<$ cannot extend $\triangleleft$. Since $\operatorname{cp}(a)$ cannot be placed, there are some clique-points placed on $\curvearrowright(a)$ or to its right. Let $\mathrm{cp}(b)$ be the leftmost among them. If $\curvearrowleft(b) \geqslant \curvearrowright(a)$, we obtain $a \triangleleft b$, which contradicts $b<a$ because $\mathrm{cp}(b)$ was placed before $\operatorname{cp}(a)$. Thus, we know that $\curvearrowleft(b)<\curvearrowright(a)$.

The clique-point $\operatorname{cp}(b)$ was not placed on the left of $\curvearrowright(a)$ because all these positions were either blocked by some other previously placed clique-points, or they are covered by some pre-drawn interval not in $P(b)$. There is at least one clique-point placed to the right of $\curvearrowleft(b)$, since otherwise we could place $\mathrm{cp}(b)$ at $\curvearrowleft(b)$ or right next to it. Let $\mathrm{cp}(c)$ be the right-most clique-point placed between $\curvearrowleft(b)$ and $\operatorname{cp}(b)$. Every point between $\mathrm{cp}(c)$ and $\curvearrowright(a)$ is covered by a pre-drawn interval not in $P(b)$. Consider the set $S$ of all the predrawn intervals not contained in $P(b)$ intersecting $[\operatorname{cp}(c), \curvearrowright(a)]$ (see the dashed intervals in Fig. 14). Let $C$ be the set of all maximal cliques containing at least one vertex from $S$. Since $S$ induces a connected subgraph, it can easily be observed that all maximal cliques of $C$ appear consecutively in $<$. We have that $a$ and $c$ belong to $C$, but $b$ does not. Since $c<b$, then $a<b$, which contradicts our original assumption that $b<a$.

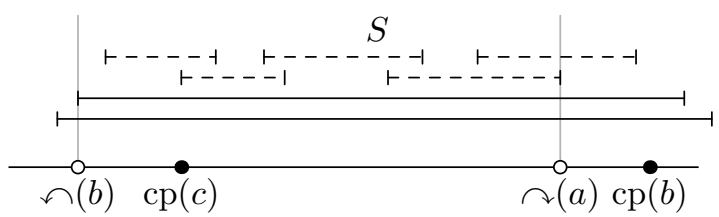

Figure 14: Illustration of the proof of Lemma 12 showing the positions of the clique-points $\operatorname{cp}(b)$ and $\operatorname{cp}(c)$; the intervals of $S$ are dashed. 
Overlaps. In this paper, we show one additional crucial property of $\triangleleft$. We say that a pair of intervals $I_{a}$ and $I_{b}$ single overlaps if $I_{a} \neq I_{b}$ and either $\curvearrowleft(a) \leqslant \curvearrowleft(b)<\curvearrowright(a) \leqslant \curvearrowright(b)$, or $\curvearrowleft(b) \leqslant \curvearrowleft(a)<\curvearrowright(b) \leqslant \curvearrowright(a)$.

Lemma 13. No pair of intervals $I_{a}$ and $I_{b}$ single overlaps.

Proof. Assume without loss of generality that $\curvearrowleft(a) \leqslant \curvearrowleft(b)$. If $\curvearrowright(a) \leqslant \curvearrowleft(b)$, then $I_{a}$ and $I_{b}$ are disjoint and do not single overlap. Suppose now that $\curvearrowleft(a) \leqslant \curvearrowleft(b)<\curvearrowright(a)$. Since all intervals of $P(a)$ cover $[\curvearrowleft(a) \curvearrowright(a)]$, we get $P(a) \subseteq P(b)$.

The position of $\curvearrowright(a)$ can be defined as a result of two distinct situations:

- If some pre-drawn interval of $P(a)$ ends in $\curvearrowright(a)$, then $\curvearrowright(b) \leqslant \curvearrowright(a)$, since the same pre-drawn interval is contained in $P(b)$.

- Otherwise, there exists a sequence of pre-drawn intervals not contained in $P(a)$ that covers the whole portion between $\curvearrowright(a)$ and the leftmost right endpoint of the intervals of $P(a)$. The left endpoints of these intervals are on or to the right of $\curvearrowright(a)$. Since the left endpoints of the intervals in $P(b)$ are to the left of $\curvearrowright(a)$, the pre-drawn intervals of the sequence are not contained in $P(b)$. Thus, $\curvearrowright(b) \leqslant \curvearrowright(a)$.

In both cases, $\curvearrowleft(a) \leqslant \curvearrowleft(b) \leqslant \curvearrowright(b) \leqslant \curvearrowright(a)$, so $I_{b}$ is contained in $I_{a}$.

If no single overlaps are allowed, every pair of intervals is either disjoint, or one interval is contained in the other (possibly the intervals are equal). This type of interval orderings is very simple and has not been much studied. We note that graphs having interval representations with no single overlaps are called trivially perfect. By examining the above proof, we get the following useful result:

Lemma 14. If $I_{a} \subseteq I_{b}$, then $P(a) \supseteq P(b)$. Further, strict containments correspond to strict inclusions.

If $I_{a}$ and $I_{b}$ are disjoint, then we only know that at least one of the sets $P(a) \backslash P(b)$ and $P(b) \backslash P(a)$ is non-empty. They both might be non-empty, or the sets $P(a)$ and $P(b)$ might be in inclusion. See Fig. 13 for examples.

Sliding Lemma. We introduce some notation. We denote by $P^{\leftrightarrow}(a)$ and $P^{\mapsto}(a)$ respectively the subsets of $P(a)$ containing the pre-drawn intervals with left-most right endpoints, and with right-most left endpoints. If $u \in P^{\leftrightarrow}(a)$ and $v \in P^{\mapsto}(a)$, then $\langle u\rangle^{\prime} \cap\langle v\rangle^{\prime}=\bigcap_{w \in P(a)}\langle w\rangle^{\prime}$, thus $I_{a}$ is a subinterval of $\langle u\rangle^{\prime} \cap\langle v\rangle^{\prime}$.

Single overlaps of pre-drawn intervals pose more constraints than containment (see Fig. $12 \mathrm{~b}$ and c). Therefore, single overlaps are more powerful in building obstructions. The following lemma states that, under some assumptions, we can turn a containment of pre-drawn intervals into a single overlap of other pre-drawn intervals; see Fig. 15.

Lemma 15 (Sliding). Let $I_{a}$ be on the left of $I_{b}, P(a) \subsetneq P(b)$ and $r \in P(b) \backslash P(a)$.

(i) There exists a pre-drawn interval $\langle z\rangle^{\prime}$ on the right of $I_{a}$ covering $r(u)$, for $u \in P^{\leftrightarrow}(a)$. Further, there exists an induced path $P_{r, z}$ from $r$ to $z$ whose vertices are all pre-drawn and not contained in $P(a)$. 
(a)

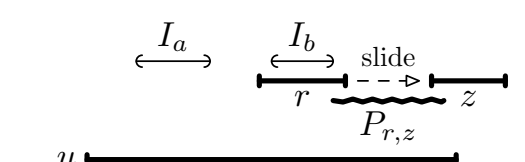

(b)

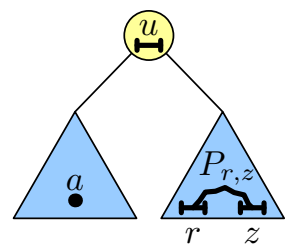

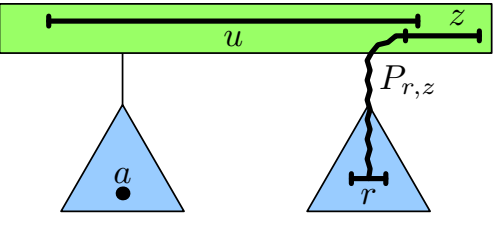

Figure 15: (a) With the assumption satisfied, we can slide $r$ to $z$ which covers $r(u)$. (b) The relative positions in the MPQ-trees of $z$ and $r$ with respect to $a$ are the same.

(ii) Consider the smallest subtree having a and the sections containing $r$. If the root of this subtree is a P-node, then $z$ and $r$ are contained in the same subtree. If the root is a $Q$-node, then $z$ and $r$ appear on the same side of $a$.

Proof. (i) By the assumptions and the definition of $I_{a}$, we get that $(\curvearrowright(a), r(u)]$ is not empty and all points in $(\curvearrowright(a), r(u)$ ] are covered by pre-drawn intervals not in $P(a)$. Among these intervals, we choose $z$ covering $r(u)$. Since $r$ is also one of these intervals, we can construct an induced path from $r$ to $z$, consisting of pre-drawn intervals not in $P(a)$.

(ii) It follows from the existence of $P_{r, z}$ not contained in $a$.

We note that possibly $r=z$. The above lemma is repeatedly used for constructing minimal obstructions. The general idea is the fact that $\langle r\rangle^{\prime}$ properly contained inside $\langle u\rangle^{\prime}$ restricts the partial representation less than $\langle z\rangle^{\prime}$ covering $r(u)$. The lemma says that we can assume that such $z$ exists and use it instead of $r$.

Flip Operation. We say that we flip the partial representation vertically when we map every $x \in \mathbb{R}$ to $-x$. This reverses the ordering $\triangleleft$. Clearly, there exists an obstruction in the original partial representation if and only if the flipped obstruction is present in the flipped partial representation. The purpose of this operation is to decrease the number of cases in the proofs.

\section{$5 \quad$ Strategy for Finding Minimal Obstructions}

In this section, we describe the general strategy to show that every non-extendible partial representation contains one of the obstructions described in Section 2.

For any two disjoint subtrees $T_{i}$ and $T_{j}$ of the MPQ-tree $T$, we write $T_{i} \triangleleft T_{j}$ if and only if there exist cliques $a \in T_{i}$ and $b \in T_{j}$ such that $a \triangleleft b$. In this situation, the maximal cliques of $T_{i}$ are forced to appear on the left of the maximal cliques of $T_{j}$.

Testing Extendibility by MPQ-tree Reordering. Recall that a MPQ-tree $T$ represents all feasible orderings of the maximal cliques of a given interval graph $G$. By Lemma 12, a partial representation is extendible if and only if there exists a reordering $T^{\prime}$ of $T$ such that the frontier of $T^{\prime}$ extends $\triangleleft$. This condition can be tested by the following algorithm (see [23]). 

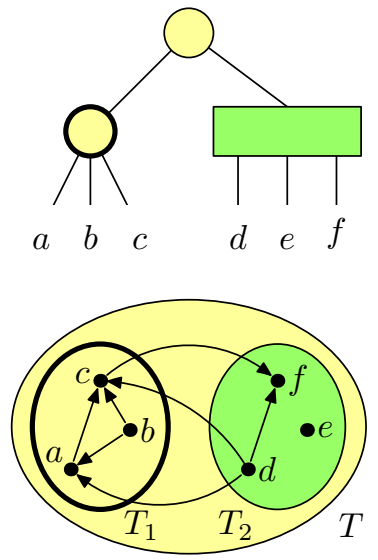
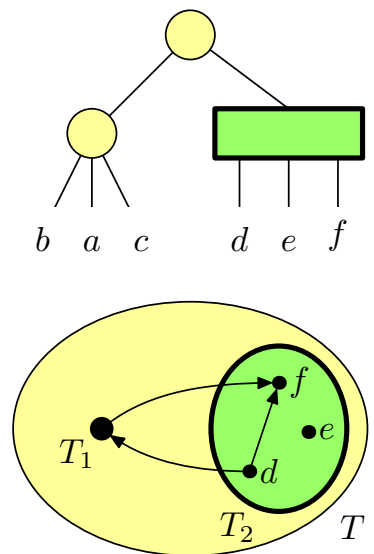
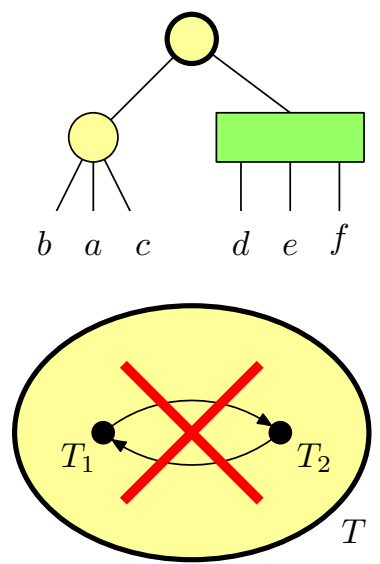

Figure 16: This example is from [23], and it shows from left to right the way in which the reordering algorithm works. We depict comparable pairs of maximal cliques by directed edges. The processed trees are contracted into vertices.

First, we reorder the highlighted P-node on the left. The subdigraph induced by $a, b$ and $c$ is ordered $b \rightarrow a \rightarrow c$. We contract this subtree $T_{1}$ into a vertex. Next, we keep the order of the highlighted Q-node and contract its subtree $T_{2}$ into a vertex. When we reorder the root $\mathrm{P}$-node, the algorithm finds a two-cycle between $T_{1}$ and $T_{2}$, and outputs "no".

We process the MPQ-tree $T$ from the bottom to the root. When a P-node is processed, we test whether there exists a linear extension of $\triangleleft$ on its subtrees. It exists if and only if $\triangleleft$ induced on the subtrees of the node is acyclic. Thus, if there is a cycle, the MPQ-tree cannot be reordered according to $\triangleleft$. When a Q-node is processed, there are two possible orderings of its subtrees, and we check whether any of them is compatible with $\triangleleft$. The partial representation is extendible if and only if all nodes can be reordered in this manner. See Fig. 16 for an example.

A node that cannot be reordered is called obstructed. A set of maximal cliques creates an obstruction if the ordering of this set in $\triangleleft$ makes the node obstructed.

Strategy. Suppose that a partial representation $\mathcal{R}^{\prime}$ is non-extendible. From [23], we know that there exists an obstructed node in the MPQ-tree. We divide the argument into three cases, according to the type of this node: an obstructed leaf (Section 6), an obstructed P-node (Section 7), and an obstructed Q-node (Section 8). Figure 17 shows an overview of the proof.

First, we argue that there exist at most three maximal cliques creating an obstruction. Then, we consider their positions in the MPQ-tree and their open intervals from the definition of $\triangleleft$. We use tools of Sections 3 and 4 to derive positions of several pre-drawn intervals forming one of the obstructions.

In Section 8.2, we prove a key tool called $k$-FAT Lemma 22: If three non-adjacent vertices $x_{k}, y_{k}$, and $z_{k}$ are pre-drawn in an order that is different from their order in the sections of a Q-node, then they induce a $k$-FAT obstruction. The proof is done by induction for $k$, and it explains why complicated obstructions are needed. 


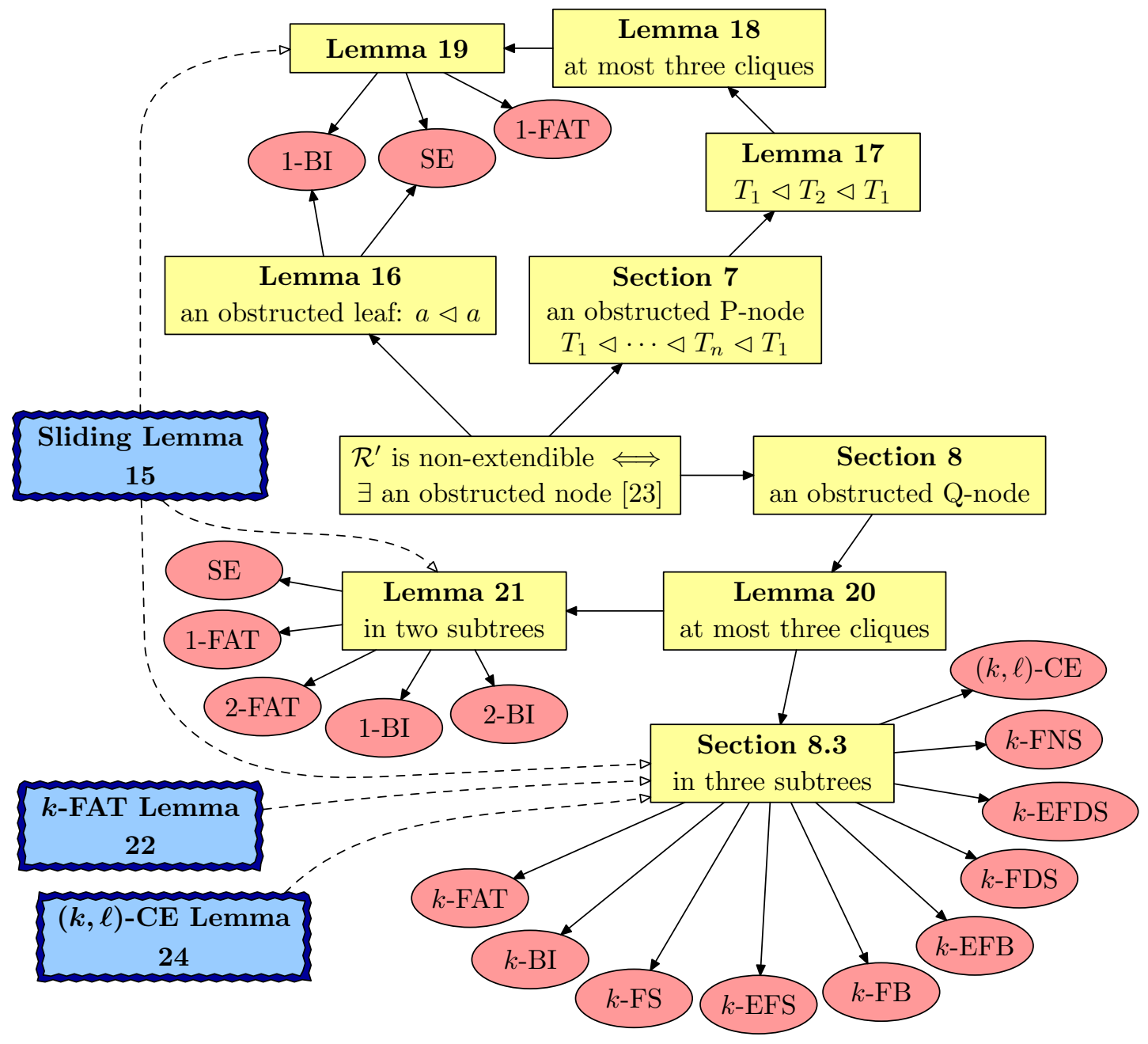

Figure 17: Overview of the main steps of the proof, it starts in the middle. The obtained obstructions are highlighted in gray, and three tools are depicted with highlighted borders. The most involved case is in Section 8.3.

\section{Obstructed Leaves}

Suppose that some clique-point $a$ cannot be placed, so $\downarrow_{a}=\emptyset$. In terms of $\triangleleft$, we get $a \triangleleft a$. Since $\triangleleft$ is a strict partial ordering, this already makes the partial representation non-extendible.

Lemma 16 (The leaf case). If a leaf is obstructed, then $G$ and $\mathcal{R}^{\prime}$ contain an SE, or 1-BI obstruction.

Proof. We name the vertices as in the definition of the 1-BI obstructions. Suppose that the leaf corresponds to a maximal clique $a$ such that $\downarrow_{a}=\emptyset$.

Let $u \in P^{\leftrightarrow}(a)$ and $v \in P^{\mapsto}(a)$ (possibly $u=v$ ). Since $I_{a}$ is a subinterval of $\bigcap_{w \in P(a)}\langle w\rangle^{\prime}$ and $\downarrow_{a}=\emptyset$, every point of $[\ell(v), r(u)]$ is covered by some pre-drawn interval 

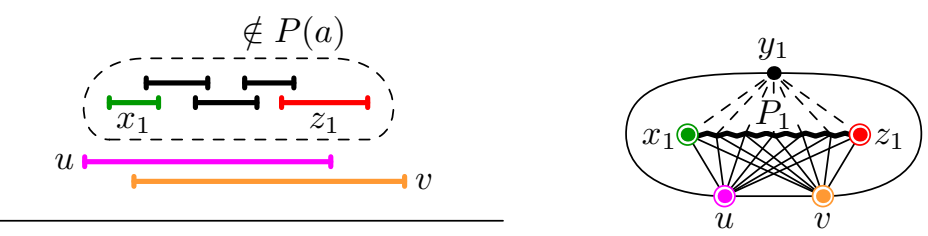

Figure 18: A construction leading to a 1-BI obstruction.

not contained in $P(a)$. Let $\left\langle x_{1}\right\rangle^{\prime}$ be one such interval covering $\ell(v)$ and let $\left\langle z_{1}\right\rangle^{\prime}$ be one such interval covering $r(u)$ (again, possibly $x_{1}=z_{1}$ ); see Fig. 18. Let $P_{1}$ be a shortest path from $x_{1}$ to $z_{1}$ consisting of pre-drawn intervals not in $P(a)$.

We prove that the relative pre-drawn position of $u, v, x_{1}$, and $z_{1}$ makes the partial representation non-extendible. The maximal clique $a$ does not contain any vertex of $P_{1}$. Since the vertices of $P_{1}$ induce a connected subgraph, by Lemma 10 there exists $y_{1} \in a$ which is non-adjacent to all vertices of $P_{1}$. Hence, these (at most) five vertices together with $P_{1}$ create either a 1-BI obstruction (when $\ell(u)<r(v)$ ), or an SE obstruction (when $\ell(u)=r(v)$, for which $x=x_{1}=z_{1}$ and we can free it). We note that this obstruction might not be minimal, in which case we can remove some vertices and get one of the minimal obstructions illustrated in Fig. 5 and 8.

\section{Obstructed P-nodes}

If a P-node is obstructed, then it has some subtrees $T_{1}, \ldots, T_{n}$ forming the cycle $T_{1} \triangleleft$ $T_{2} \triangleleft \cdots \triangleleft T_{n} \triangleleft T_{1}$. We start by showing that the specific structure of $\triangleleft$ forces the existence of a two-cycle, so we can assume that $n=2$.

Lemma 17. If a P-node is obstructed, then it has two subtrees $T_{1}$ and $T_{2}$ such that $T_{1} \triangleleft T_{2} \triangleleft T_{1}$.

Proof. The proof is illustrated in Fig. 19a. Let $T_{1} \triangleleft \cdots \triangleleft T_{n} \triangleleft T_{1}$ be a shortest cycle for the obstructed P-node. To get a contradiction, we assume $n \geqslant 3$. Since $T_{1} \triangleleft T_{2}$, there exist $a \in T_{1}$ and $b \in T_{2}$ such that $a \triangleleft b$. Similarly, there exist $c \in T_{2}$ and $d \in T_{3}$ such that $c \triangleleft d$. We know that $I_{a}$ is on the left of $I_{b}$, and $I_{c}$ is on the left of $I_{d}$. We analyze the remaining relative positions.

First, $I_{d}$ is not on the right of $I_{a}$, since otherwise $T_{1} \triangleleft T_{3}$ and a shorter cycle would exist. Additionally, $I_{d}$ is not on the left of $I_{b}$, since we would get $T_{3} \triangleleft T_{2}$, and $T_{2}$ and $T_{3}$ would form a two-cycle. According to Lemma 13, no single overlaps of open intervals are allowed, so $I_{d}$ necessarily contains both $I_{a}$ and $I_{b}$; see Fig. 19a. Therefore, $I_{c}$ is on the left of $I_{a}$, so $T_{2} \triangleleft T_{1}$ and we get a two-cycle.

To create a two-cycle, at most four cliques are enough. Aside from Lemma 13, so far we have not used that $\triangleleft$ arises from a partial interval representation. Next, we use properties of the MPQ-tree.

Lemma 18. A two-cycle $T_{1} \triangleleft T_{2} \triangleleft T_{1}$ is created by at most three maximal cliques. 

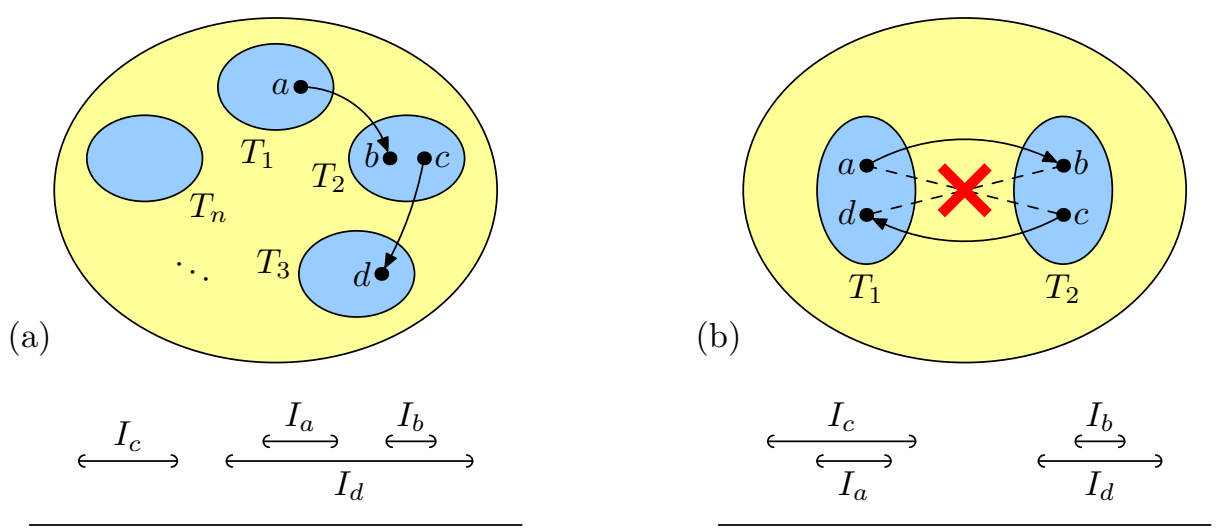

Figure 19: (a) At the top, a shortest $n$-cycle of $\triangleleft$ on the children of a P-node. At the bottom, the derived positions of the open intervals. (b) At the top, the four cliques involved in a two-cycle in $\triangleleft$. The cliques $a$ and $c$ are incomparable, and so are $b$ and $d$. At the bottom, one of the four possible configurations of the open intervals.

Proof. The proof is depicted in Fig. 19b. Suppose that this two-cycle is given by four cliques $a, d \in T_{1}$ and $b, c \in T_{2}$ such that $a \triangleleft b$ and $c \triangleleft d$. Assume for contradiction that no three of these cliques define the two-cycle, i.e., $a$ and $c$ are incomparable, and so are $b$ and $d$. According to Lemma 13, $I_{a} \subseteq I_{c}$ or $I_{a} \supseteq I_{c}$, and analogously for $I_{b}$ and $I_{d}$. In all of the four cases, $I_{c}$ is on the left of $I_{b}$, and $I_{d}$ is on the right of $I_{a}$.

We look at the case where $I_{a} \subseteq I_{c}$ and $I_{b} \subseteq I_{d}$, as in Fig. 19b. By Lemma 14, we have $P(c) \subseteq P(a)$. Therefore, $P(c)$ contains no vertices from the sections of $T_{2}$. Similarly, $P(d) \subseteq P(b)$, and $P(d)$ contains no vertices from the sections of $T_{1}$. Therefore $P(c)=P(d)$, which implies $I_{c}=I_{d}$, a contradiction. The other cases can be analyzed similarly.

It remains to put these results together and characterize the possible obstructions.

Lemma 19 (The P-node case). If a P-node is obstructed, then $G$ and $\mathcal{R}^{\prime}$ contain an $S E$, 1-FAT, or 1-BI obstruction.

Proof. According to Lemma 17, the obstructed P-node has a two-cycle in $\triangleleft$. By Lemma 18, there are at most three maximal cliques defining this cycle. First assume that this cycle is defined by two cliques $a \in T_{1}, b \in T_{2}$ such that $a \triangleleft b \triangleleft a$. According to the definition of $\triangleleft$, this implies that $I_{a}=I_{b}$, both of lenght zero. Therefore $P(a)=P(b)$. Let $u \in P^{\leftrightarrow}(a)$ and $v \in P^{\mapsto}(a)$ (possibly $u=v$ ); we have that $\langle u\rangle^{\prime} \cap\langle v\rangle^{\prime}$ is a singleton. Since $a$ and $b$ are two maximal cliques, there exists $x \in a \backslash b$ and $y \in b \backslash a$. We get an SE obstruction.

It remains to deal with the case where three cliques define the two-cycle. Let $a, c \in T_{1}$ and $b \in T_{2}$ such that $a \triangleleft b \triangleleft c$. We have three non-intersecting intervals whose left-toright order is $I_{a}, I_{b}$ and $I_{c}$. Since $I_{a}$ and $I_{c}$ are disjoint, one of the sets $P(a) \backslash P(c)$ and $P(c) \backslash P(a)$ is non-empty. Without loss of generality, we assume that $P(a) \backslash P(c) \neq \emptyset$. 
(a)
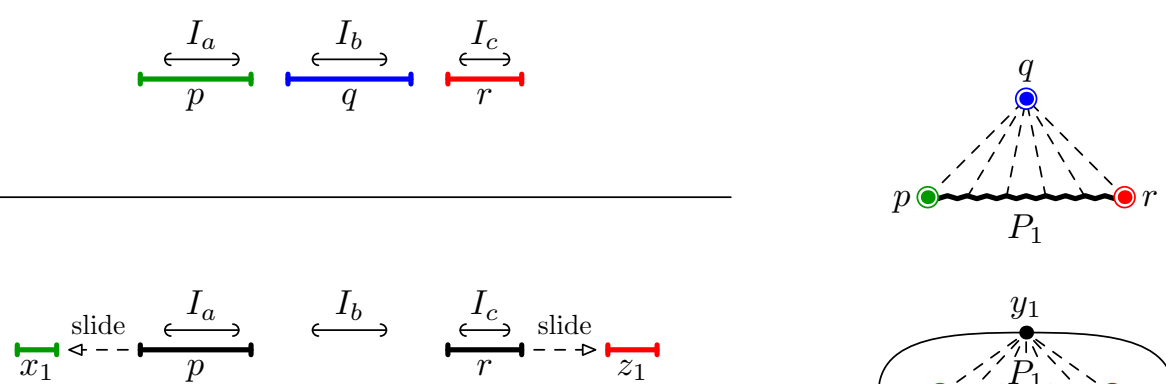

(b)
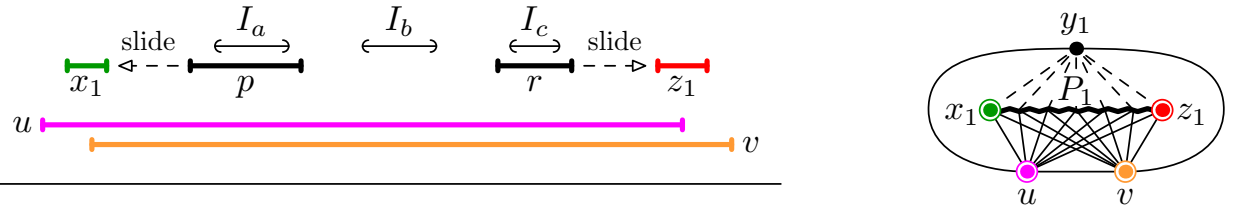

Figure 20: The two cases of the proof of Lemma 19. (a) Case 1 leads to a 1-FAT obstruction. (b) Case 2 leads to a 1-BI obstruction.

Let $p \in P(a) \backslash P(c)$; then $p$ belongs to sections of $T_{1}$, and as a consequence $p \notin P(b)$. Therefore $\langle p\rangle^{\prime}$ is on the left of $I_{b}$. We distinguish two cases.

Case 1: $P(b) \backslash P(c) \neq \emptyset$. We choose $q \in P(b) \backslash P(c)$. Then $q$ belongs to sections of $T_{2}$, and $\langle q\rangle^{\prime}$ is between $\langle p\rangle^{\prime}$ and $I_{c}$. In the next paragraph we show that there also exists $r \in P(c) \backslash P(b)$. Then $r$ belongs to sections of $T_{1}$, and $\langle r\rangle^{\prime}$ is on the right of $\langle q\rangle^{\prime}$, as in Fig. 20a. By Lemma 8(ii), $G\left[T_{1}\right]$ is connected; let $P_{1}$ be a shortest path from $p$ to $r$ in $G\left[T_{1}\right]$. We obtain a 1-FAT obstruction for $x_{1}=p, y_{1}=q$ and $z_{1}=r$.

It remains to show that such $r$ exists. Suppose for contradiction that $P(c) \backslash P(b)=\emptyset$. Since $P(c) \subsetneq P(b)$, no vertex of $P(c)$ appears in sections of $T_{1}$, and we get $P(c) \subsetneq P(a)$. Consequently, every pre-drawn interval of $P(c)$ contains $[\curvearrowleft(a), \curvearrowright(c)]$. The position of $I_{c}$ implies that every point of $[\curvearrowleft(a), \curvearrowleft(c))$ is covered by some pre-drawn interval not contained in $P(c)$. In particular, there exists a path from $p$ to $q$ consisting of such intervals. Since $p$ belongs to sections of $T_{1}$ and $q$ belongs to sections of $T_{2}$, every path from $p$ to $q$ contains a vertex of the section of the P-node, or of a section above it; hence, the path contains a vertex belonging to $c$. We obtain a contradiction.

Case 2: $P(b) \backslash P(c)=\emptyset$. Then there exists $r \in P(c) \backslash P(b)$. We again observe that $\langle r\rangle^{\prime}$ is on the right of $I_{b}$, as depicted in Fig. 20b. Furthermore, $P(b) \subseteq P(a) \cap P(c)$, so every pre-drawn interval of $P(b)$ contains $[\curvearrowleft(a), \curvearrowright(c)]$.

We construct a 1-BI obstruction and we name the vertices as in the definition. Let $u \in P^{\leftrightarrow}(b)$ and $v \in P^{\mapsto}(b)$ (possibly $u=v$ ). Since $p$ does not necessarily cover $\ell(v)$ and $r$ does not necessarily cover $r(u)$, we might not be able to construct a 1-BI obstruction with $x_{1}=p$ and $z_{1}=q$. We instead use Sliding Lemma 15. By applying it (flipped) to $I_{b}, I_{a}$ and $p$, we obtain a pre-drawn interval $x_{1}$ covering $\ell(v)$ (possibly $x_{1}=p$ ). By applying it to $I_{b}, I_{c}$ and $r$, we obtain a pre-drawn interval $z_{1}$ covering $r(u$ ) (possibly $\left.z_{1}=r\right)$. Furthermore, $x_{1}$ and $z_{1}$ belong to sections of $T_{1}$. Since $G\left[T_{1}\right]$ is connected by Lemma 8(ii), there exists a shortest path $P_{1}$ from $x_{1}$ to $z_{1}$ containing no vertex of $b$. By Lemma 10, there exists $y_{1} \in b$ non-adjacent to all vertices of $P_{1}$. We obtain a 1-BI obstruction. 


\section{Obstructed Q-nodes}

Suppose that a Q-node with subtrees $T_{1}, \ldots, T_{n}$ is obstructed. Then the two possible orderings of this Q-node are not compatible with $\triangleleft$. Notice that at most four cliques are sufficient to create the obstruction. We next prove that at most three cliques are already sufficient.

Lemma 20. If a Q-node is obstructed, there exists an obstruction created by at most three maximal cliques.

Proof. Suppose that an obstruction is created by four cliques $a \in T_{\alpha}, b \in T_{\beta}, c \in T_{\gamma}$ and $d \in T_{\delta}$ such that $\alpha<\beta, \gamma<\delta, a \triangleleft b$, and $c \triangleright d$. We know that $I_{a}$ is on the left of $I_{b}$, and $I_{c}$ is on the right of $I_{d}$. Notice that the four subtrees $T_{\alpha}, T_{\beta}, T_{\gamma}$ and $T_{\delta}$ are not necessarily distinct. We classify all possible orderings $<$ of $\alpha, \beta, \gamma, \delta$ in two general cases, namely, $\alpha \neq \gamma$ and $\alpha=\gamma$. In the first case, we may assume without loss of generality that $\alpha<\gamma$.

Case 1: $\alpha<\gamma<\delta$ (see Fig. 21a). Consider the relative positions of $I_{c}$ and $I_{d}$ with respect to $I_{a}$. If $I_{d}$ is to the left of $I_{a}$, we have $d \triangleleft a \triangleleft b$, and these three cliques already create an obstruction. If $I_{c}$ is to the right of $I_{a}$, then we get $a \triangleleft c$ and $c \triangleright d$, creating an obstruction. If neither happens, then $I_{c}$ and $I_{d}$ are subintervals of $I_{a}$. Thus $c, d \triangleleft b$. If $\beta \leqslant \gamma$, we have $a \triangleleft b$ and $b \triangleright d$, creating an obstruction. If $\beta>\gamma$, then $d \triangleleft c \triangleleft b$, which also creates an obstruction.

Case 2: $\alpha=\gamma$ (see Fig. 21b). If $I_{c}$ does not intersect $I_{b}$, or $I_{d}$ does not intersect $I_{a}$, it is easy to see that three of the cliques already create an obstruction. Suppose next that these intersections occur. Then $d \triangleleft b$. If $\delta<\beta$ or $\beta<\delta$, it is again easy to show that three cliques are enough to create an obstruction. It only remains to consider the case where $\alpha=\gamma<\beta=\delta$.

Since the intervals $I_{c}$ and $I_{a}$ are non-intersecting, we may assume without loss of generality that there exists $x \in P(a) \backslash P(c)$. This vertex $x$ belongs to sections of $T_{\alpha}$. Thus $x \notin P(d)$, and we get that $I_{a} \subsetneq I_{d}$. By Lemma 14, $P(d) \subsetneq P(a)$; in particular, $P(d)$ contains no private pre-drawn interval from sections of $T_{\beta}$, and all pre-drawn intervals of $s_{\beta}(Q)$ are also contained in $s_{\alpha}(Q)$.

(a)

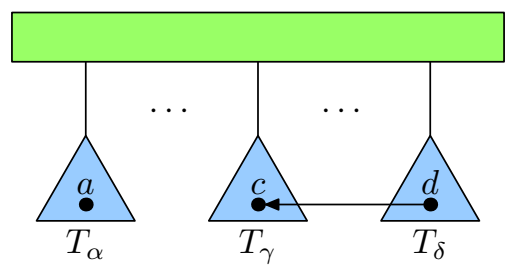

$$
\stackrel{I_{a}}{\stackrel{I_{d}}{\hookrightarrow} \stackrel{I_{c}}{\hookrightarrow}} \stackrel{I_{b}}{\longleftrightarrow}
$$

(b)

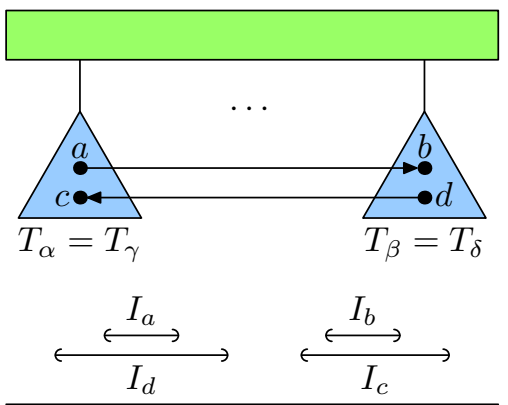

Figure 21: Two cases of the proof of Lemma 20. The Q-node is depicted in the top, while in the bottom we have the relative positions of the intervals. 
Since $P(d) \backslash P(b)=\emptyset$, there exists $y \in P(b) \backslash P(d)$ which is contained in sections of $T_{\beta}$. We next apply the argument in the previous paragraph, and obtain $y \notin P(c), I_{b} \subsetneq I_{c}$, and $P(c) \subsetneq P(b)$. Consequently, $P(c)$ contains no private pre-drawn intervals from sections of $T_{\alpha}$, and all pre-drawn intervals of $s_{\alpha}(Q)$ are contained in $s_{\beta}(Q)$. We conclude that $P(c)=P(d)$ and $I_{c}=I_{d}$, which gives a contradiction.

In summary, we can assume that a minimal obstruction involves at most three maximal cliques. These three cliques belong to either two or three different subtrees.

In the rest of the section, many figures describe positions of derived pre-drawn intervals in sections of the Q-node and its subtrees; for instance Fig. 22. Some of these intervals necessarily belong to sections of the Q-node, since they belong to maximal cliques of several subtrees; for instance $t_{2}$ in Fig. 22. But for the remaining intervals, it is not important to distinguish whether they belong to sections of the Q-node or one of its subtrees, only their relative positions in the Q-node matter; for instance $q$ and $x_{1}$ in Fig. 22 .

\subsection{Cliques in Two Different Subtrees}

In this section, we deal with the case where the maximal cliques belong to two different subtrees.

Lemma 21 (The Q-node case, Two Subtrees). If at most three cliques creating the obstruction belong to two different subtrees, then $G$ and $\mathcal{R}^{\prime}$ contain an SE, 1-FAT, 2-FAT, 1-BI, or 2-BI obstruction.

Proof. The proof is similar to that of Lemma 19. If two maximal cliques create an obstruction, we can argue as in the first paragraph of the proof of Lemma 19, and we obtain an SE obstruction. It remains to deal with the case of three maximal cliques $a, b$, and $c$.

We can assume that $a \triangleleft b \triangleleft c$ and that, for some $i<j$, we have $a, c \in T_{i}$ and $b \in T_{j}$. Furthermore, without loss of generality, there exist $p \in P(a) \backslash P(c)$. Since $p$ belongs to sections of $T_{i}$, then $p \notin P(b)$, and thus $\langle p\rangle^{\prime}$ lies to the left of $I_{b}$. We distinguish two cases.

Case 1: $P(b) \backslash P(c) \neq \emptyset$. Then there exists $q \in P(b) \backslash P(c)$ such that $\langle q\rangle^{\prime}$ lies between $\langle p\rangle^{\prime}$ and $I_{c}$. Since $q$ is non-adjacent to $p$, it belongs to sections of either $Q$ or $T_{j}$. Notice that in any case $s_{q}^{\leftarrow}(Q)$ is on the right of $s_{i}(Q)$. Arguing as in Case 1 of the proof of Lemma 19, we observe that there exists $r \in P(c) \backslash P(b)$. Furthermore, it follows that $\langle r\rangle^{\prime}$ lies to the right of $\langle q\rangle^{\prime}$; see Fig. 22a on the left.

If there exists a path $P_{1}$ from $p$ to $r$ avoiding $N[q]$, we get a 1-FAT obstruction for $x_{1}=p, y_{1}=q, z_{1}=r$ and $P_{1}$. By Lemma 7 , we know that $s_{i}(Q) \neq s_{q}^{\leftarrow}(Q)$. If $s_{i}(Q) \nsubseteq s_{q}^{\leftarrow}(Q)$, then there exists some $w \in s_{i}(Q) \backslash s_{q}^{\leftarrow}(Q)$. Therefore, $P_{1}=$ pwr is such a path. It remains to deal with the case where no such path $P_{1}$ exists, which implies that $s_{i}(Q) \subsetneq s_{q}^{\leftarrow}(Q)$; see Fig. 22a on the right.

Consider the set $W=s_{i}(Q)$. Let $t_{2}$ be a vertex of $W$ whose section $\overrightarrow{t_{2}}(Q)$ is leftmost. Let $C$ be the component of $G[Q] \backslash W$ containing $q$. Since $s_{q}^{\leftarrow}(Q) \backslash W$ is non-empty, $C$ consists of the vertices of at least two subtrees of the Q-node. If $t_{2}$ was adjacent to all 
(a)

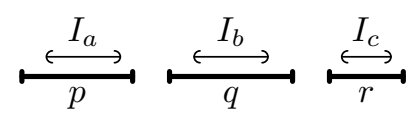

(b)

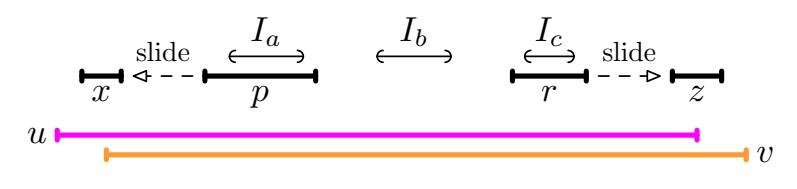

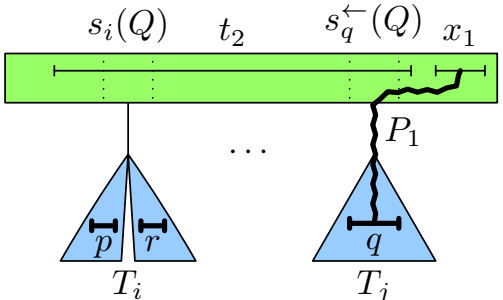

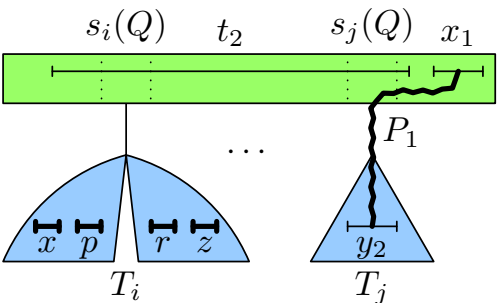

Figure 22: (a) Case 1: The pre-drawn intervals and the situation in the MPQ-tree for $s_{i}(Q) \subsetneq s_{q}^{\leftarrow}(Q)$. (b) Case 2: The pre-drawn intervals and the situation when there exists no path from $x$ to $z$ avoiding the vertices of $b$.

vertices of $C$, it would be possible to flip the ordering of this component, contradicting the fact that there are only two possible orderings for $Q$. Therefore, $t_{2}$ is not adjacent to all vertices of $C$. We choose $x_{1} \in C \backslash N\left[t_{2}\right]$ whose section $s_{x_{1}}^{\leftarrow}(Q)$ is leftmost. Let $P_{1}$ be a shortest path from $q$ to $x_{1}$ whose inner vertices are adjacent to $t_{2}$. It follows that $x_{2}=p$, $y_{2}=q, z_{2}=r, P_{2}=x_{2} t_{2}, t_{2}, x_{1}$, and $P_{1}$ define a 2-FAT obstruction. (By Lemma 9, all inner vertices of $P_{1}$ are adjacent to $t_{2}$.)

Case 2: $P(b) \backslash P(c)=\emptyset$. Then there exists $r \in P(c) \backslash P(b)$. Since $\langle r\rangle^{\prime}$ lies on the right of $I_{b}$, the vertex $r$ is not contained in $a$ and it belongs to sections of $T_{i}$. We use the same approach as in Case 2 of the proof of Lemma 19. Since $P(b) \subseteq P(a) \cap P(c)$, every pre-drawn interval of $P(b)$ covers $[\curvearrowleft(a), \curvearrowright(c)]$. Let $u \in P^{\leftrightarrow}(b)$ and $v \in P^{\mapsto}(b)$ (possibly $u=v)$.

By applying Sliding Lemma 15 twice, we get $x, z \notin P(b)$ such that $\langle x\rangle^{\prime}$ covers $\ell(v)$ and $\langle z\rangle^{\prime}$ covers $r(u)$; see Fig. $22 \mathrm{~b}$ on the left.

Suppose that there exists a path $P_{x, z}$ from $x$ to $z$ avoiding all vertices of $b$. Let $x_{1}=x$, $z_{1}=z$, and $P_{1}$ be a shortest path from $x_{1}$ to $z_{1}$ in $G[Q] \backslash b$. By Lemma 10, there exists $y_{1} \in b$ non-adjacent to $P_{1}$. We obtain a 1 -BI obstruction.

Suppose next that there is no path $P_{x, z}$ avoiding $b$. We know that $x$ and $z$ belong to sections of $T_{i}$, since there exist paths $P_{x, p}$ and $P_{r, z}$ avoiding $b$, from the above applications of Sliding Lemma 15. Since no path $P_{x, z}$ avoiding $b$ exists, we have $s_{i}(Q) \subsetneq s_{j}(Q)$. As in Case 1 , let $W=s_{i}(Q)$, and let $t_{2}$ be a vertex of $W$ whose section $\overrightarrow{t_{t_{2}}}(Q)$ is leftmost (possibly $t_{2}=u$ or $t_{2}=v$ ). We again infer that $t_{2}$ is not adjacent to all vertices of $C$, where $C$ is the component of $G[Q] \backslash W$ containing $b \backslash W$. We choose $x_{1} \in C \backslash N\left[t_{2}\right]$ whose section $s_{x_{1}}^{\leftarrow}(Q)$ is leftmost. Since $s_{i}(Q) \subsetneq s_{j}(Q) \subseteq b$, there exists $y_{2} \in b$ non-adjacent to $x$ and $z$. We get a 2-BI obstruction for $x_{2}=x, y_{2}, z_{2}=z, u, v$, a shortest path $P_{1}$ from $y_{2}$ to $x_{1}$ in $C$, and $P_{2}=x_{2} t_{2}$. (By Lemma 9 , all inner vertices of $P_{1}$ are adjacent to $t_{2}$.) 


\section{$8.2 k$-FAT and $(k, \ell)$-CE Lemmas}

In this section, we give two tools for the case, analyzed in Section 8.3, where the three maximal cliques creating the obstruction belong to three different subtrees. These tools give insight into the structure of the Q-nodes, and explain the way in which complex obstructions such as $k$-FAT and $(k, \ell)$-CE obstructions are formed.

$\boldsymbol{k}$-FAT Lemma. First, we present a useful lemma that allows to locate $k$-FAT obstructions. The key idea of the proof is similar to Case 1 of the proof of Lemma 21, but applied inductively for $k$.

Lemma 22 ( $\boldsymbol{k}$-FAT). Let $Q$ be a $Q$-node with children $T_{1}, \ldots, T_{n}$, and let $a, b$ and $c$ be three cliques of $T[Q]$ contained respectively in $T_{\alpha}, T_{\beta}$ and $T_{\gamma}$, for $\alpha<\beta<\gamma$. Let $x_{k} \in P(a), y_{k} \in P(c)$ and $z_{k} \in P(b)$ be three disjoint pre-drawn intervals such that $\left\langle y_{k}\right\rangle^{\prime}$ is between $\left\langle x_{k}\right\rangle^{\prime}$ and $\left\langle z_{k}\right\rangle^{\prime}$. Then $G[Q]$ and $\mathcal{R}^{\prime}\left[\left\{x_{k}, y_{k}, z_{k}\right\}\right]$ contain a $k$-FAT obstruction.

Proof. The proof, illustrated in Fig. 23, is by induction. We always denote the vertices as in the definition of $k$-FAT obstructions. If we find a 1-FAT or 2-FAT obstruction, the statement is true. Otherwise, we recurse on a smaller part of the Q-node, where we find a structure identical to a $(k-1)$-FAT obstruction, except for the fact that the vertex $x_{k-1}$ is free. Together with some vertices in the remainder of the Q-node, we obtain a $k$-FAT obstruction. We next provide the details.

Let $k$ be some yet unspecified integer, determined by the recursion. We want to argue that $G[Q]$ contains a $k$-FAT obstruction because the ordering of $\left\langle x_{k}\right\rangle^{\prime},\left\langle y_{k}\right\rangle^{\prime}$ and $\left\langle z_{k}\right\rangle^{\prime}$ is incorrect (in every representation, $\left\langle z_{k}\right\rangle$ is between $\left\langle x_{k}\right\rangle$ and $\left\langle y_{k}\right\rangle$ ). Suppose that there exists a path from $x_{k}$ to $z_{k}$ whose inner vertices are non-adjacent to $y_{k}$. Then we obtain a 1-FAT obstruction. It remains to deal with the harder situation where no such path exists.

Let $C\left(x_{k}\right)$ be the connected component of $G[Q] \backslash N\left[y_{k}\right]$ containing $x_{k}$. By our assumption, $z_{k} \notin C\left(x_{k}\right)$. We denote by $W_{k}$ the subset of $N\left[y_{k}\right]$ containing those vertices that are adjacent to some vertex of $C\left(x_{k}\right)$; see Fig. 23, middle. Notice that the vertices of $C\left(x_{k}\right)$ appear only in sections and subtrees to the left of $s_{\beta}(Q)$. Therefore, every vertex of $W_{k}$ lies in the sections of $Q$ and stretches from the left of $s_{\beta}(Q)$ to $s_{\gamma}(Q)$; see Fig. 23, right. In other words, $W_{k} \subseteq s_{\beta}(Q) \cap s_{\gamma}(Q)$ and every vertex of $W_{k}$ is adjacent to $z_{k}$.
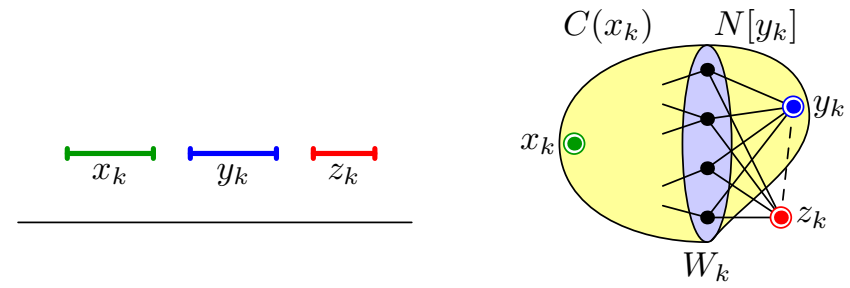

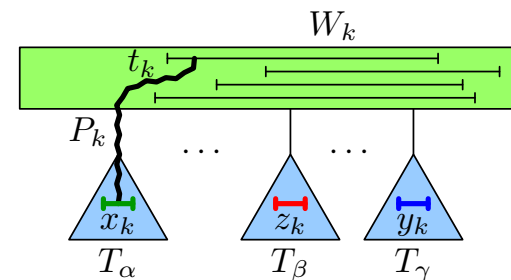

Figure 23: On the left, the position of the pre-drawn intervals. In the middle, the construction of $W_{k} \subsetneq N\left[y_{k}\right]$ in $G[Q]$. On the right, the Q-node with the three subtrees and the intervals of $W_{k}$ depicted in its sections.

THE EleCtronic JOURnAl of COMBinatorics 25(4) (2018), \#P4.55 
(a)

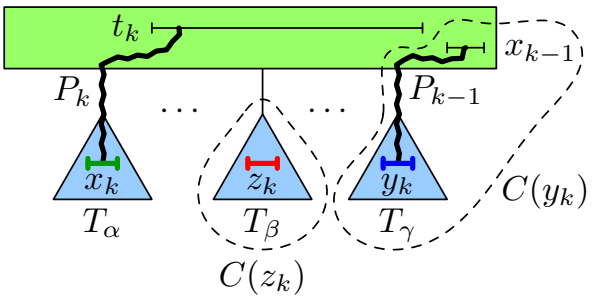

(b)

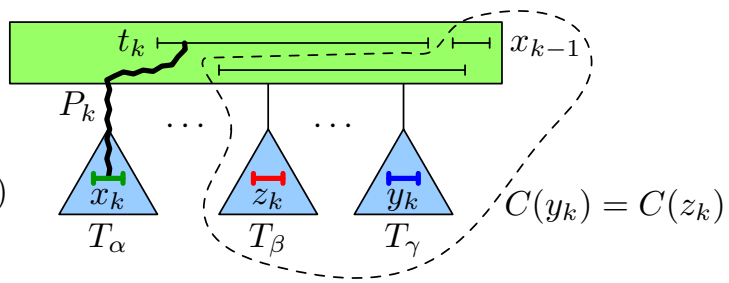

Figure 24: (a) In Case 1, there exists a path $P_{k-1}$ from $x_{k-1}$ to $y_{k}$ whose inner vertices avoid $z_{k}$. (b) In Case 2, we have $C\left(y_{k}\right)=C\left(z_{k}\right)$ and such a path might no longer exist. For instance, every path from $x_{k-1}$ to $y_{k}$ in $C\left(y_{k}\right)$ might use the depicted interval in the sections of $Q$, which is also adjacent to $z_{k}$.

Let $C$ be a connected component of $G[Q] \backslash W_{k}$. If $C$ contains a vertex from some section of $Q$, we call it big. Notice that in this situation $C$ has a vertex contained in two consecutive sections of $Q$ and their subtrees. Otherwise, $C$ consists of some vertices of a subtree of $Q$, and we call it small. The section above a subtree containing a small component is a subset of $W_{k}$. Additionally, if two small components are placed in two different subtrees, the two sections above these subtrees are different.

The graph $G[Q] \backslash W_{k}$ is disconnected, as $x_{k}$ and $z_{k}$ belong to different components. Let us denote the connected component containing $y_{k}$ by $C\left(y_{k}\right)$, and the one containing $z_{k}$ by $C\left(z_{k}\right)$. Let $t_{k}$ be a vertex of $W_{k}$ whose section $\vec{s}_{t_{k}}(Q)$ is leftmost. Let $P_{k}$ be a shortest path from $x_{k}$ to $t_{k}$ in $G\left[C\left(x_{k}\right) \cup\left\{t_{k}\right\}\right]$; see Fig. 23, right. We distinguish two cases.

Case 1: $C\left(y_{k}\right) \neq C\left(z_{k}\right)$. This case is very similar to the proof of Lemma 21; see Fig. 24a. Every vertex of $W_{k}$ is adjacent to some vertex of $C\left(x_{k}\right)$ and to some vertex of $C\left(y_{k}\right)$. Therefore, it is also adjacent to every vertex of $C\left(z_{k}\right)$. If $C\left(z_{k}\right)$ was big, then we could reverse its sections in the Q-node, contradicting the fact that there are only two possible orderings for a Q-node. Therefore, $C\left(z_{k}\right)$ is small. Notice that then $C\left(y_{k}\right)$ is not small, since otherwise we would get $s_{\beta}(Q)=W_{k}=s_{\gamma}(Q)$, contradicting Lemma 7. Thus, $C\left(y_{k}\right)$ is big.

Let us set $y_{k-1}=z_{k}$ and $z_{k-1}=y_{k}$. The vertex $t_{k}$ is not universal for $C\left(y_{k}\right)$; otherwise, every vertex of $W_{k}$ would be universal and this would give additional orderings of $C\left(y_{k}\right)$ in $Q$. Let $x_{k-1}$ be a vertex of $C\left(y_{k}\right) \backslash N\left[t_{k}\right]$ whose section $s_{x_{k-1}}^{\leftarrow}(Q)$ is leftmost. Notice that $s_{x_{k-1}}^{\leftarrow}(Q)$ is always the next section to $\overrightarrow{t_{k}}(Q)$. Let $P_{k-1}$ be a shortest path from $x_{k-1}$ to $z_{k-1}$ in $C\left(y_{k}\right)$. By Lemma 9 , all inner vertices of $P_{k-1}$ are adjacent to $t_{k}$. Since this path lies in $C\left(y_{k}\right)$, the inner vertices are non-adjacent to $y_{k-1}, x_{k}$ and $P_{k}$. We have constructed a 2-FAT obstruction.

Case 2: $C\left(y_{k}\right)=C\left(z_{k}\right)$. In this case, the component $C\left(y_{k}\right)$ is big; see Fig. 24b. Therefore, similarly as above, $t_{k}$ is not universal for $C\left(y_{k}\right)$. We put $y_{k-1}=z_{k}$ and $z_{k-1}=y_{k}$. We choose $x_{k-1} \in C\left(y_{k}\right) \backslash N\left[t_{k}\right]$ in the same way as in Case 1 . Notice that $x_{k-1}$ is a non-neighbor of $y_{k-1}$, since otherwise it would be a neighbor of $t_{k}$. On the other hand, $x_{k-1}$ might be adjacent to $z_{k-1}$ or not. If it is, we get a 2-FAT obstruction for $k=2$ with $P_{1}=x_{k-1} z_{k-1}$. If it is not, we proceed as follows.

As before, every shortest path from $x_{k-1}$ to $z_{k-1}$ has all inner vertices adjacent to 
$t_{k}$. Since all vertices of $C\left(y_{k}\right)$ are non-adjacent to $x_{k}$ and the inner vertices of $P_{k}$, every shortest path satisfies this as well. There exists a shortest path from $x_{k-1}$ to $z_{k-1}$ in $C\left(y_{k}\right)$, but we cannot guarantee that the inner vertices of this path are non-adjacent to $y_{k-1}$. We solve this issue by applying the entire argument of the proof recursively to $C\left(y_{k}\right)$.

In every representation extending the partial representation, the intervals of $C\left(x_{k}\right)$ form a connected subset of the real line placed to the left of $\left\langle y_{k}\right\rangle^{\prime}$. Therefore, $\left\langle t_{k}\right\rangle$ stretches from $C\left(x_{k}\right)$ to $\left\langle z_{k}\right\rangle^{\prime}$, covering $\left\langle y_{k}\right\rangle^{\prime}$. Thus $\left\langle x_{k-1}\right\rangle$ is placed to the right of $\left\langle z_{k}\right\rangle^{\prime}=\left\langle y_{k-1}\right\rangle^{\prime}$ in every extending representation (see Fig. 6b). Again, $\left\langle y_{k-1}\right\rangle^{\prime}$ has to be placed between $\left\langle x_{k-1}\right\rangle$ and $\left\langle z_{k-1}\right\rangle^{\prime}$. We assume that $\left\langle x_{k-1}\right\rangle$ is pre-drawn on the right of $\left\langle y_{k-1}\right\rangle^{\prime}$ and repeat the same argument for $C\left(y_{k}\right)$ and the MPQ-tree restricted to these vertices. The role of $x_{k}, y_{k}$ and $z_{k}$ is played by $x_{k-1}, y_{k-1}$ and $z_{k-1}$, respectively. (The ordering of the pre-drawn intervals is flipped.)

The paragraphs above show the induction step of our proof (by induction on, say, the number of considered sections of $Q)$. By the induction hypothesis, we find a $(k-1)$-FAT obstruction. By making $x_{k-1}$ free and adding $x_{k}, t_{k}$ and $P_{k}$, we get a $k$-FAT obstruction in the original partial representation. Clearly $t_{k}$ is adjacent to the entire $(k-1)$-FAT obstruction with the exception of $x_{k-1}$, since all further vertices are contained in a section to the left of $s_{x_{k-1}}^{\leftarrow}(Q)$. The reason is that we always use shortest paths which are $Q$ monotone by Lemma 9. By the same reason, they are non-adjacent to the inner vertices of $P_{k}$ and to $x_{k}$, as required.

To make the argument complete, we should check that all the assumptions used throughout the proof apply recursively, in particular the arguments concerning nonuniversality of $t_{k-1}$ and reversing big components. This is true because both components $C\left(y_{k-1}\right)$ and $C\left(z_{k-1}\right)$ of $C\left(y_{k}\right) \backslash W_{k-1}$ appear to the left of $x_{k-1}$, so $t_{k}$ and the other vertices of $W_{k}$ are universal for them. This property is preserved throughout the recursion, so $C\left(y_{\ell}\right)$ and $C\left(z_{\ell}\right)$ are adjacent to all vertices of $W_{k}, W_{k-1}, \ldots, W_{\ell+1}$. Similarly, the rest of the inductive proof can be formalized.

The above proof shows that the structure of a Q-node can be highly complicated, leading to complicated obstructions such as $k$-FAT. Actually, $k$-FAT Lemma 22 is a very useful tool because it can be also applied in situations where not all $x_{k}, y_{k}$, and $z_{k}$ are pre-drawn, to build other obstructions. Fig. 25 shows an example.

Lemma 23. Consider a $k$-FAT obstruction $H_{k}$ for $k>2$. If we swap the positions of $\left\langle x_{k}\right\rangle^{\prime}$ and $\left\langle y_{k}\right\rangle^{\prime}$, then we obtain a new obstruction which contains a 1-FAT obstruction for $x_{1}^{\prime}=y_{k}, y_{1}^{\prime}=x_{k}$, and $z_{1}^{\prime}=z_{k}$. Further, if $k=2$ and this does not happen, then $x_{2}$ is adjacent to $t_{2}$.

Proof. For $k \geqslant 3$, the graph $H_{k} \backslash N\left[x_{k}\right]$ is connected; in particular, there exists a path $P_{1}^{\prime}=y_{k} t_{k-1} z_{k}$ avoiding $N\left[x_{k}\right]$. For $k=2$, there exists the path $P_{1}^{\prime}=y_{2} t_{2} z_{2}$ avoiding $N\left[x_{2}\right]$, unless $x_{2}$ is adjacent to $t_{2}$.

$(\boldsymbol{k}, \boldsymbol{\ell})$-CE Lemma. Suppose that we have the situation in Fig. 26. We can easily show that it yields to a $(k, \ell)$-CE obstruction: 

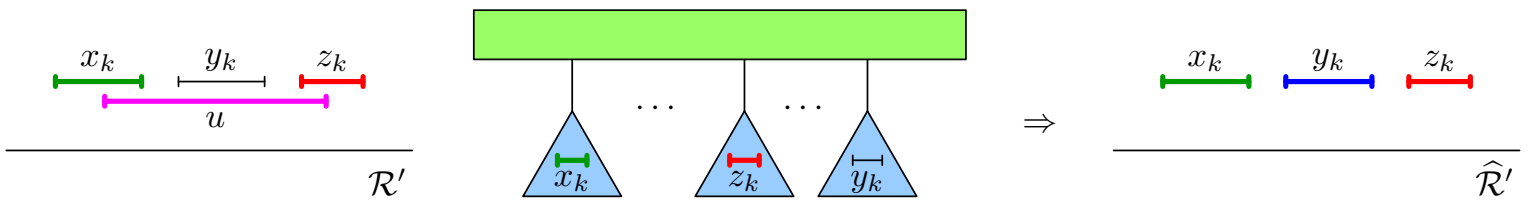

Figure 25: Suppose that we show that a partial representation $\mathcal{R}^{\prime}$ has three pre-drawn intervals as on the left, and that there is a vertex $y_{k}$ adjacent to $u$ and non-adjacent to $x_{k}$ and $z_{k}$. Then $\left\langle y_{k}\right\rangle$ has to be placed between $\left\langle x_{k}\right\rangle^{\prime}$ and $\left\langle z_{k}\right\rangle^{\prime}$ in every extending representation. Thus, we can assume it is pre-drawn there and obtain a modified partial representation $\widehat{\mathcal{R}}^{\prime}$. If we further show that $x_{k}, y_{k}$ and $z_{k}$ are placed in appropriate sections of $G[Q]$ for some Q-node $Q$, we can apply $k$-FAT Lemma 22 and we get a $k$-FAT obstruction in $G[Q]$ and $\widehat{\mathcal{R}}^{\prime}\left[\left\{x_{k}, y_{k}, z_{k}\right\}\right]$. Together with $\langle u\rangle^{\prime}$, this forms a $k$-BI obstruction in $G$ and $\mathcal{R}^{\prime}$.

Lemma $24((\boldsymbol{k}, \boldsymbol{\ell})-\mathrm{CE})$. Let $Q$ be a $Q$-node with children $T_{1}, \ldots, T_{n}$, and let $a, b$ and $c$ be three cliques of $T[Q]$ contained respectively in $T_{\alpha}, T_{\beta}$ and $T_{\gamma}$, for $\alpha<\beta<\gamma$. Let $x_{k} \in a, y_{k} \in c$ and $z_{k} \in P(b)$ be three non-adjacent vertices having a common pre-drawn neighbor $u$ such that $\langle u\rangle^{\prime}$ single overlaps $\left\langle z_{k}\right\rangle^{\prime}$. Then $G[Q] \cup\{u\}$ and $\mathcal{R}^{\prime}\left[\left\{z_{k}, u\right\}\right]$ contain $a(k, \ell)-C E$ obstruction, where either $\ell=1$ or $k=\ell=2$.

Proof. By applying $k$-FAT Lemma 22 twice, once when $\left\langle x_{k}\right\rangle$ is on the left of $\left\langle y_{k}\right\rangle$ and once when it is on the right, we obtain the $(k, \ell)$-CE obstruction. Further, by Lemma 23, we get that either $\ell=1$, or $k=\ell=2$.

The proof of the following lemma reveals the structure of the minimal $(k, \ell)-\mathrm{CE}$ obstructions in detail:

Lemma 25. For $2 \geqslant k \geqslant \ell$, the list of minimal $(k, \ell)-C E$ obstructions is given in Fig. 9 . For $k \geqslant 3$, the minimal $(k, \ell)-C E$ obstructions have $\ell=1$ and consist of the graph $H_{k}$ together with a vertex $u$, either adjacent to all vertices of $H_{k}$, or $u=t_{k}$.

Proof. The simplest case is when there exist a path $P_{k}$ from $x_{k}$ to $z_{k}$ avoiding $N\left[y_{k}\right]$, and a path $P_{\ell}^{\prime}$ from $y_{k}$ to $z_{k}$ avoiding $N\left[x_{k}\right]$. Let $P_{k}$ and $P_{\ell}^{\prime}$ be shortest such paths as in Fig. 26, right. We get a $(1,1)$-CE obstruction. By Lemma 9, the paths $P_{k}$ and $P_{\ell}^{\prime}$ are monotone. Therefore, their inner vertices are non-adjacent to each other, with the possible exception of the last vertices before $z_{k}$, which can be adjacent or even identical.
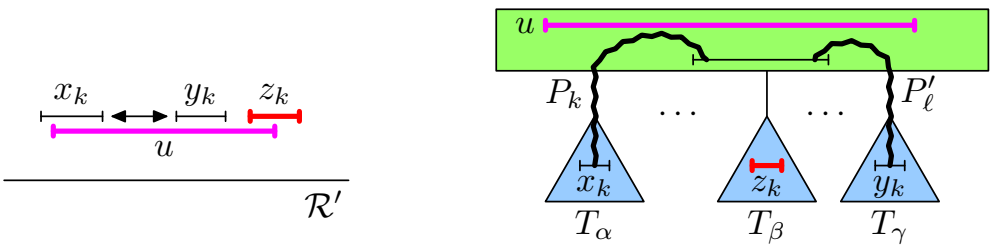

Figure 26: When $\langle u\rangle^{\prime}$ single overlaps $\left\langle z_{k}\right\rangle^{\prime}$, and the vertices $x_{k}, y_{k}$, and $z_{k}$ are placed in the MPQ-tree as on the right, we get a $(k, \ell)$-CE obstruction. 
(a)

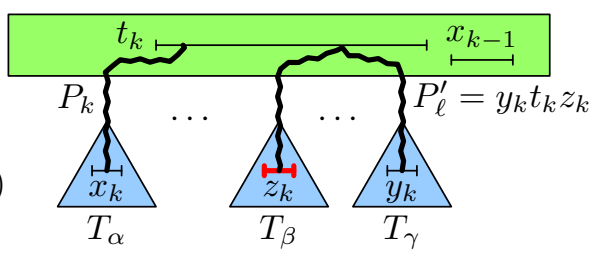

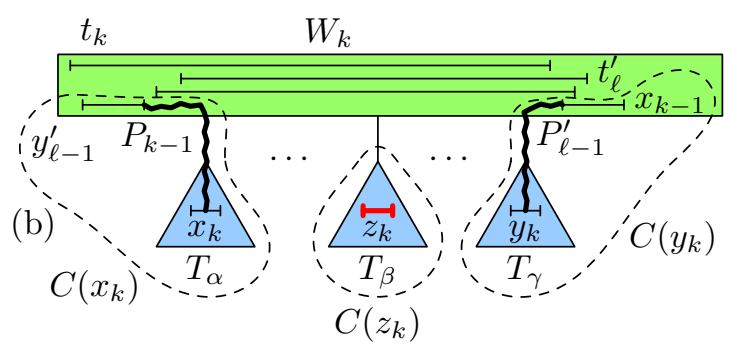

Figure 27: (a) Case 1: If there exists a path $P_{\ell}^{\prime}$ from $y_{k}$ to $z_{k}$ avoiding $N\left[x_{k}\right]$, then we get a $(k, 1)$-CE obstruction. (b) Case 2 : We get a $(2,2)$-CE obstruction.

Concerning minimality, we can always find one of the three finite $(1,1)$-CE obstructions depicted in Fig. 9a. The reason is that when paths $P_{k}$ and $P_{\ell}^{\prime}$ are long, we can take as $x_{k}$ and $y_{k}$ one of their inner vertices, making them shorter.

Suppose next that there exists no path $P_{k}$ from $x_{k}$ to $z_{k}$ avoiding $N\left[y_{k}\right]$. Assuming that $\left\langle x_{k}\right\rangle$ is placed on the left of $\left\langle y_{k}\right\rangle$, we apply $k$-FAT Lemma 22 and we get the subgraph $H_{k}$ of a $k$-FAT obstruction (which is not the complete $k$-FAT obstruction because $x_{k}$ and $y_{k}$ are free). Let $C\left(x_{k}\right), W_{k}$, and $t_{k}$ be defined as in the proof of $k$-FAT Lemma 22 .

Case 1: There exists some path $P_{\ell}^{\prime}$ from $y_{k}$ to $z_{k}$ avoiding $N\left[x_{k}\right]$. Let $P_{\ell}^{\prime}$ be a shortest such path (notice that $\ell=1$ ). Together with the above subgraph $H_{k}$, we get a $(k, 1)$-CE obstruction; see Fig. 27a. In particular, if some vertex $w \in W_{k}$ is non-adjacent to $x_{k}$ (possibly $w=t_{k}$ ), we can use $P_{\ell}^{\prime}=y_{k} w z_{k}$.

We note that when $k \geqslant 3$, such a path $P_{\ell}^{\prime}$ necessarily exists, as we can use $P_{\ell}^{\prime}=$ $y_{k} t_{k-1} z_{k}$, as argued in Lemma 23. Therefore, the $(k, 1)$-CE obstructions consist of the subgraph $H_{k}$ together with $u$; assuming minimality, we have that either $u$ is adjacent to all vertices of $H_{k}$, or $u=t_{k}$. If $k=2$, then $P_{\ell}^{\prime}$ might still exist but it might be longer and might use inner vertices not contained in $H_{k}$. Concerning minimality, we always find one of the three $(2,1)$-CE obstructions depicted in Fig. 9b. Indeed, $P_{2}$ can be assumed to be of length one or two, since otherwise we could use one of its inner vertices as $x_{2}$. For length two, we get $P_{1}^{\prime}=y_{k} t_{k} z_{k}$. For length one, we get a path $P_{1}^{\prime}$ from $z_{2}$ to $y_{2}$, and we can assume that $y_{2}$ is adjacent to $x_{1}$ (otherwise we could use as $y_{2}$ the neighbor of $x_{1}$ on $\left.P_{1}\right)$.

Case 2: No such path $P_{\ell}^{\prime}$ exists. By Lemma 23, necessarily $k=2$. We want to show that there exists a $(2,2)$-CE obstruction which we describe in detail.

Notice that all vertices $w \in W_{k}$ are adjacent to $x_{k}, y_{k}$, and $z_{k}$, since otherwise there would exist a path $P_{\ell}^{\prime}=y_{k} w z_{k}$ avoiding $N\left[x_{k}\right]$. Hence the vertices of $W_{k}$ belong to sections of $Q$, covering all subtrees between $T_{\alpha}$ and $T_{\gamma}$; see Fig. 27b. Let $C\left(y_{k}\right)$ and $C\left(z_{k}\right)$ be the components of $G[Q] \backslash W_{k}$ containing $y_{k}$ and $z_{k}$, respectively. Since there exists no path $P_{\ell}^{\prime}$, we obtain that $C\left(x_{k}\right), C\left(y_{k}\right)$, and $C\left(z_{k}\right)$ are pairwise different. To determine the structure of a $(2,2)$-CE obstruction, we apply the argument from Case 1 of the proof of $k$-FAT Lemma 22 symmetrically twice.

Let $t_{k}$ be a vertex of $W_{k}$ having leftmost section $\overrightarrow{s_{k}}(Q)$ and let $t_{\ell}^{\prime}$ be a vertex of $W_{k}$ having rightmost section $s_{t_{\ell}^{\prime}}^{\leftarrow}(Q)$ (possibly $t_{k}=t_{\ell}^{\prime}$ ). It is easy to see that $C\left(z_{k}\right)$ is small, 
otherwise we could flip it and obtain an ordering of the maximal cliques not compatible with the Q-node.

Similarly as in the proof of $k$-FAT Lemma 22, this implies that both $C\left(x_{k}\right)$ and $C\left(y_{k}\right)$ are big. Therefore, $t_{k}$ is not universal for $C\left(y_{k}\right)$ and $t_{\ell}^{\prime}$ is not universal for $C\left(x_{k}\right)$. As in the proof of $k$-FAT Lemma 22, we choose $x_{k-1} \in C\left(y_{k}\right)$ non-adjacent to $t_{k}$ and $y_{\ell-1}^{\prime} \in C\left(x_{k}\right)$ non-adjacent to $t_{\ell}^{\prime}$. There exist paths $P_{k-1}$ from $x_{k-1}$ to $y_{k}$ and $P_{\ell-1}^{\prime}$ from $y_{\ell-1}^{\prime}$ to $x_{k}$. In consequence, we obtain a $(2,2)$-CE obstruction.

Regarding minimality, notice that we can assume that $y_{2}$ is adjacent to $x_{1}$, and $x_{2}$ is adjacent to $y_{1}^{\prime}$; otherwise, we could choose as $y_{2}$ and $x_{2}$ the neighbors of $x_{1}$ and $y_{1}^{\prime}$ on the paths $P_{1}$ and $P_{1}^{\prime}$, respectively. We get the four minimal finite $(2,2)$-CE obstructions that are illustrated in Fig. 9c.

\subsection{Cliques in Three Different Subtrees}

When a Q-node $Q$ is obstructed by three maximal cliques $a \in T_{\alpha}, b \in T_{\beta}$ and $T_{\gamma}$, where $\alpha<\beta<\gamma$, the situation is quite complex. Fig. 28 gives an overview of the cases and obstructions obtained in this case.

Lemma 26. Without loss of generality, we can assume that $a \triangleleft b \triangleright c$ and $\curvearrowright(a) \leqslant \curvearrowright(c)$.

Proof. Since $a, b$ and $c$ create an obstruction, $b$ is either a minimal or a maximal element in $\left.\triangleleft\right|_{\{a, b, c\}}$. Without loss of generality (using the flip operation), we can assume that $b$ is maximal, so $a \triangleleft b \triangleright c$. Since we can swap $a$ and $c$ by reversing the Q-node, we can assume that $\curvearrowright(a) \leqslant \curvearrowright(c)$.

Since $a \triangleleft b \triangleright c$, both $I_{a}$ and $I_{c}$ appear on the left of $I_{b}$. Since $\curvearrowright(a) \leqslant \curvearrowright(c)$, either $I_{a}$ contained in $I_{c}$, or $I_{a}$ is on the left of $I_{c}$. The first case is easier:

Lemma 27. If $I_{a}$ is contained in $I_{c}$, then $G$ and $\mathcal{R}^{\prime}$ contain a $(k, \ell)-C E$ obstruction, where $\ell=1$ or $2 \geqslant k \geqslant \ell$.

Proof. The proof is illustrated in Fig. 29. By Lemma 14, $P(c) \subseteq P(a)$. Since $b$ is placed between $a$ and $c$ in the Q-node $Q$, every vertex contained in both $a$ and $c$ is contained in $b$ as well. Hence $P(c) \subsetneq P(b)$, and there exists $r \in P(b) \backslash P(c)$. Since $\langle r\rangle^{\prime}$ is on the right of $I_{c}$, it is also on the right of $I_{a}$, and thus $r \notin P(a)$.

Let $u \in P^{\leftrightarrow}(c)$. We apply Sliding Lemma 15 to $I_{c}, I_{b}$, and $\langle r\rangle^{\prime}$. We get a pre-drawn interval $\left\langle z_{k}\right\rangle^{\prime}$ covering $r(u)$, and an induced path $P_{r, z_{k}}$ from $r$ to $z_{k}$ consisting of pre-drawn intervals not in $P(c)$. Therefore $z_{k}$ is on the left of $c$ in $Q$. Since all pre-drawn intervals of $P_{r, z_{k}}$ do not belong to $P(c)$, they are on the right of $I_{c}$. Thus they are also on the right of $I_{a}$, which implies that they do not belong to $P(a)$. Consequently, $z_{k}$ is between $a$ and $c$ in $Q$.

Let $x_{k} \in a$ and $y_{k} \in c$ be vertices non-adjacent to $z_{k}$. By $(k, \ell)$-CE Lemma $24, x_{k}, y_{k}$, $z_{k}$, and $u$ create a $(k, \ell)$-CE obstruction, for $\ell=1$ or $2 \geqslant k \geqslant \ell$. Notice that the clique associated to $z_{k}$ is some $b^{\prime} \neq b$. 


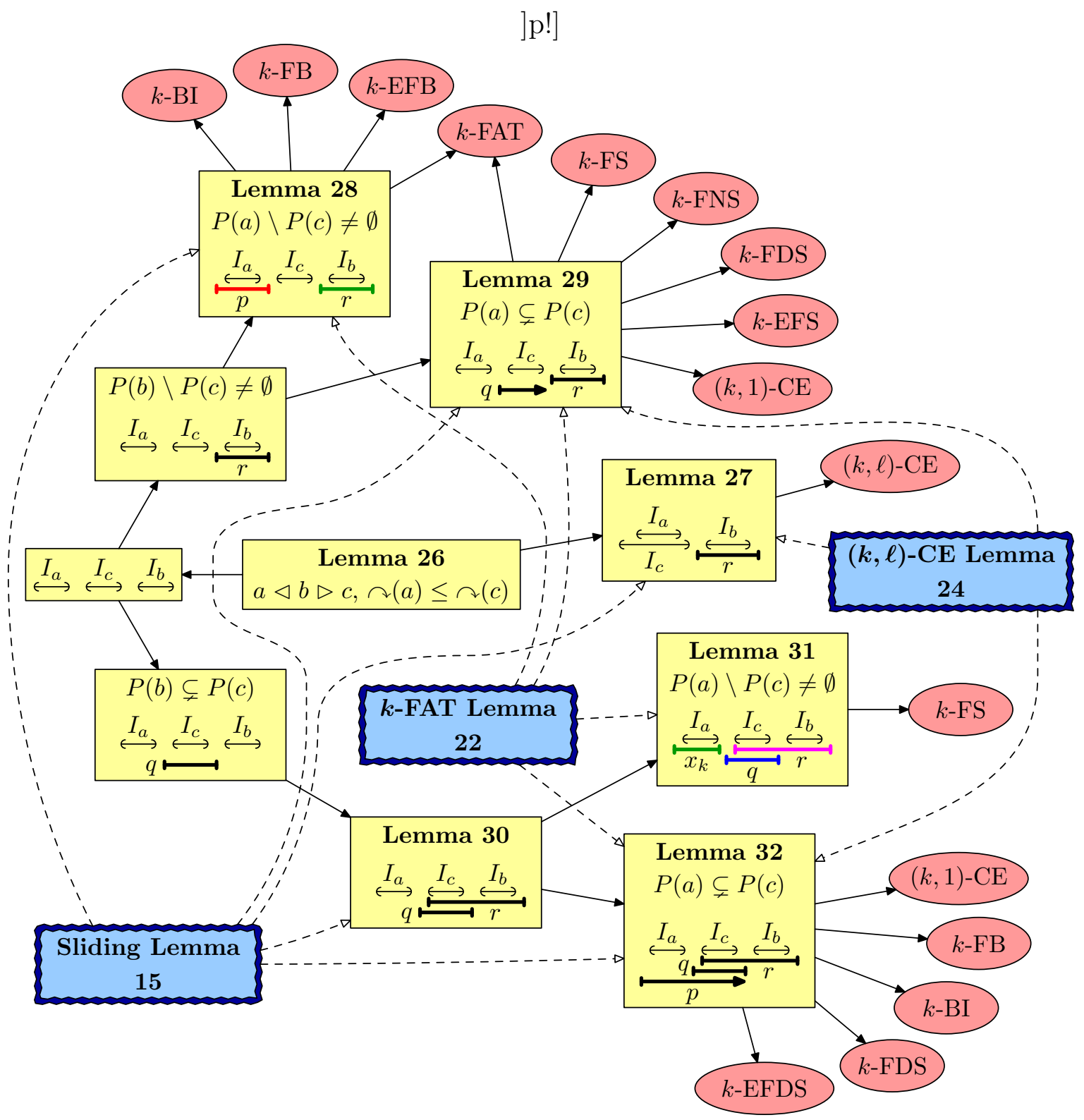

Figure 28: A summary of Section 8.3. The diagram starts in the middle with Lemma 26. Inside the cases, we draw the positions of $I_{a}, I_{b}, I_{c}$, and some pre-drawn intervals. An arrow at a pre-drawn interval means that it may be further stretched in the given direction. The obtained obstructions are highlighted in gray, the used tools have highlighted borders. 

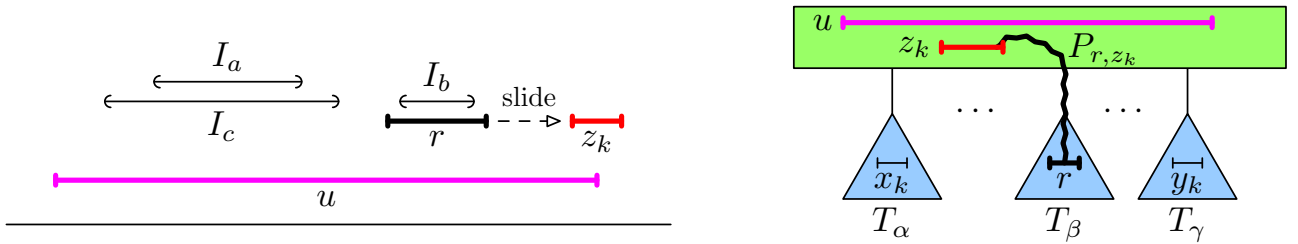

Figure 29: Proof of Lemma 27. On the left, the pre-drawn intervals. On the right, their positions in the MPQ-tree.

The case where $I_{a}$ is on the left of $I_{c}$ is further divided into several subcases. In the next two lemmas, we focus on the situation where $P(b) \backslash P(c) \neq \emptyset$.

Lemma 28. If $I_{a}$ is on the left of $I_{c}, P(b) \backslash P(c) \neq \emptyset$ and $P(a) \backslash P(c) \neq \emptyset$, then $G$ and $\mathcal{R}^{\prime}$ contain a $k$-FAT, $k$-BI $(k \leqslant 2), k-F B$, or $k$-EFB obstruction.

Proof. The proof is illustrated in Fig. 30. Let $p \in P(a) \backslash P(c)$ and $r \in P(b) \backslash P(c)$. Then $\langle p\rangle^{\prime}$ is on the left of $I_{c}$, and $\langle r\rangle^{\prime}$ is on the right of $I_{c}$. Clearly, $\langle p\rangle^{\prime}$ and $\langle r\rangle^{\prime}$ are disjoint, so $p$ appears in the Q-node on the left of $r$. Let $u \in P^{\leftrightarrow}(c)$ and $v \in P^{\mapsto}(c)$ (possibly $u=v$ ).

If $r(u) \leqslant r(r)$, then $z_{k}=r$. Obviously, $z_{k}$ is between $p$ and $c$ in the Q-node. Otherwise, $P(c) \subsetneq P(b)$, and we apply Sliding Lemma 15 to $I_{c}, I_{b}$, and $r$. We obtain a pre-drawn interval $\left\langle z_{k}\right\rangle^{\prime}$ not contained in $P(c)$ covering $r(u)$, and a path $P_{r, z_{k}}$ whose inner vertices are pre-drawn and not contained in $P(c)$. Notice that all of these pre-drawn vertices are on the right of $I_{c}$. Therefore, these vertices are not contained in $P(a)$. In this case, we also get that $z_{k}$ is between $p$ and $c$ in the Q-node.

Similarly, if $\ell(p) \leqslant \ell(v)$, then $x_{k}=p$. Otherwise, we use the flipped version of Sliding Lemma 15 to $I_{c}, I_{a}$, and $p$, which gives a pre-drawn interval $\left\langle x_{k}\right\rangle^{\prime}$ not contained in $P(c)$ covering $\ell(v)$. By a similar argument, in both cases, we show that $x_{k}$ is on the left of $z_{k}$ in the Q-node.

Let $y_{k} \in c$ be a vertex non-adjacent to $z_{k}$ (possibly, $y_{k}=u$ or $y_{k}=v$ ). Such a vertex exists because $z_{k}$ is on the left of $c$ in the Q-node. Notice that $y_{k}$ is also non-adjacent to $x_{k}$. Since $y_{k}$ is adjacent to $u$ and $v$, in every extending representation $\left\langle y_{k}\right\rangle$ is between $\left\langle x_{k}\right\rangle^{\prime}$ and $\left\langle z_{k}\right\rangle^{\prime}$. So we can assume that it is pre-drawn in this position and, by $k$-FAT Lemma 22, we get a $k$-FAT obstruction. Together with $u$ and $v$ (or possibly only one of them), we get one of the obstructions in Fig. 31.
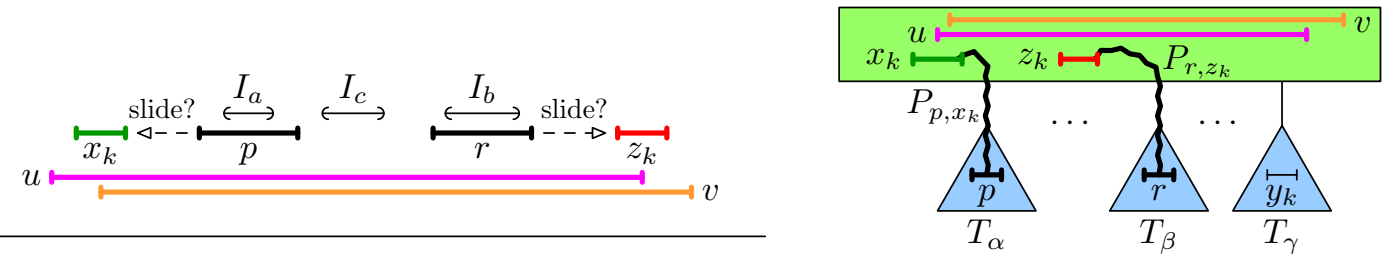

Figure 30: Proof of Lemma 28. On the left, the pre-drawn intervals, with possible sliding on each side. On the right, their positions in the MPQ-tree. 
(a)

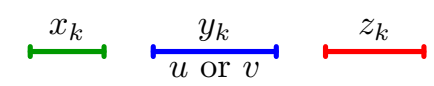

(c)

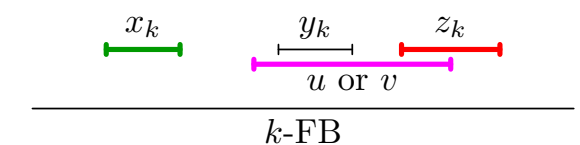

(b)

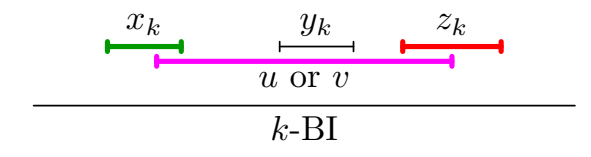

(d)

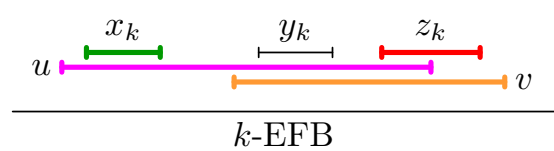

(e)

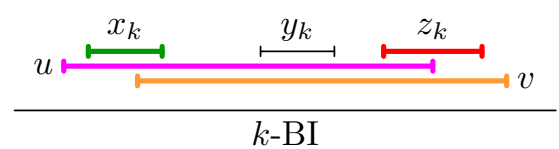

Figure 31: The different obstructions obtained in the proof of Lemma 28. If $\ell\left(x_{k}\right) \leqslant$ $\ell(u) \leqslant r(u) \leqslant r\left(z_{k}\right)$, we get one of the obstructions (a) to (c). Since $z_{k}$ is in the Q-node between $x_{k}$ and $c$, if $u$ or $v$ intersect $x_{k}$, then they also intersect $z_{k}$. In the cases (d) and $(\mathrm{e}), \ell(u)<\ell\left(x_{k}\right)$ and $r\left(z_{k}\right)<r(v)$. Since $u$ intersects $z_{k}$, there are only two possible obstructions.

Lemma 29. If $I_{a}$ is on the left of $I_{c}, P(b) \backslash P(c) \neq \emptyset$ and $P(a) \subsetneq P(c)$, then $G$ and $\mathcal{R}^{\prime}$ contain a $k$-FAT, $(k, 1)-C E, k-F S, k-F D S, k-F N S$, or $k$-EFS obstruction.

Proof. We choose $r \in P(b) \backslash P(c)$ and $q \in P(c) \backslash P(a)$ with leftmost right endpoint. Then $\langle r\rangle^{\prime}$ is on the right of $I_{c}$ and $\langle q\rangle^{\prime}$ is on the right of $I_{a}$. We note that $q$ might be adjacent to $r$ or not, and might belong to $P(b)$ or not. Since $P(a) \subsetneq P(c)$, we get from the structure of the Q-node that also $P(a) \subsetneq P(b)$. Let $u \in P^{\leftrightarrow}(a)$. Notice that at least one of $q$ and $u$ belongs to $P^{\leftrightarrow}(c)$.

Case 1: $u \in P^{\leftrightarrow}(c)$. Then $r(u) \leqslant r(q)$ and $P(c) \subsetneq P(b)$; the situation is depicted in Fig. 32a. We apply Sliding Lemma 15 to $I_{c}, I_{b}$, and $r$. We get a pre-drawn interval $z_{k} \notin P(c)$ covering $r(u)$, and a path $P_{r, z_{k}}$ consisting of pre-drawn intervals not contained in $P(c)$. Therefore, $z_{k}$ is on left of $c$ in the Q-node. Since $I_{a}$ is on the left of $I_{c}$, all vertices of $P_{r, z_{k}}$ are also not contained in $P(a)$. Thus $z_{k}$ is on the right of $a$ in the Q-node.

Choose $y_{k} \in c$ non-adjacent to $z_{k}$. By Lemma 10, there exists $x_{k} \in a$ non-adjacent to both $z_{k}$ and $q$. Since $z_{k}$ is between $a$ and $c$ in the Q-node, also $x_{k}$ is non-adjacent to $y_{k}$. Since $y_{k} q z_{k}$ is a path avoiding $N\left[x_{k}\right]$, by $(k, \ell)$-CE Lemma 24 we obtain a $(k, 1)$-CE obstruction.

Case 2: $q \in P^{\leftrightarrow}(c)$. Then $r(q)<r(u)$. First we argue that, without loss of generality, we can assume that either $\langle q\rangle^{\prime}$ and $\langle r\rangle^{\prime}$ are disjoint, or $\langle r\rangle^{\prime}$ covers $r(q)$. Suppose that $\langle r\rangle^{\prime}$ is contained in $\langle q\rangle^{\prime}$. Since $q \in P^{\leftarrow}(c)$, this implies that $P(c) \subsetneq P(b)$. By applying Sliding Lemma 15 to $I_{c}, I_{b}$ and $r$, we obtain a pre-drawn interval $\widetilde{r}$ not in $P(c)$ which covers $r(q)$. We also get a path $P_{r, \widetilde{r}}$ whose vertices are pre-drawn and not contained in $P(c)$; since $I_{a}$ is on the left of $I_{c}$, they are also not in $P(a)$. Therefore $\widetilde{r}$ is between $a$ and $c$ in the Q-node. Further, $\widetilde{r}$ belongs to some clique $\widetilde{b}$ for which $I_{\widetilde{b}}$ is on the right of $I_{c}$. From now on, we work with $\widetilde{r}$ as $r$, and with $\widetilde{b}$ as $b$. Hence the assumption on the relative positions of $\langle q\rangle^{\prime}$ and $\langle r\rangle^{\prime}$ holds. 
(a)

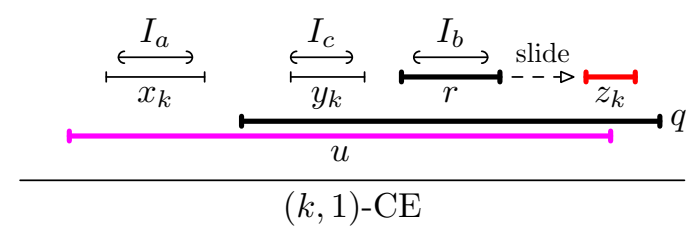

(b)

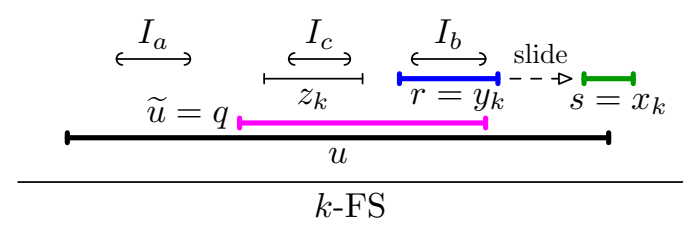

(c)

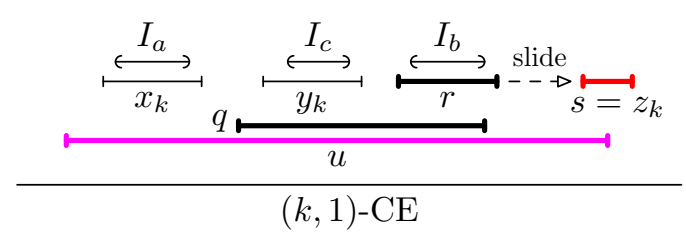

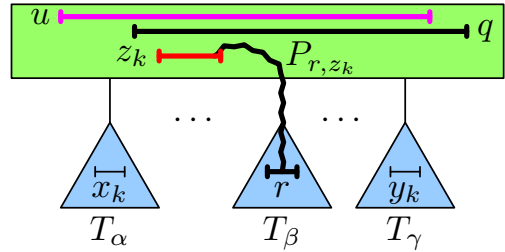
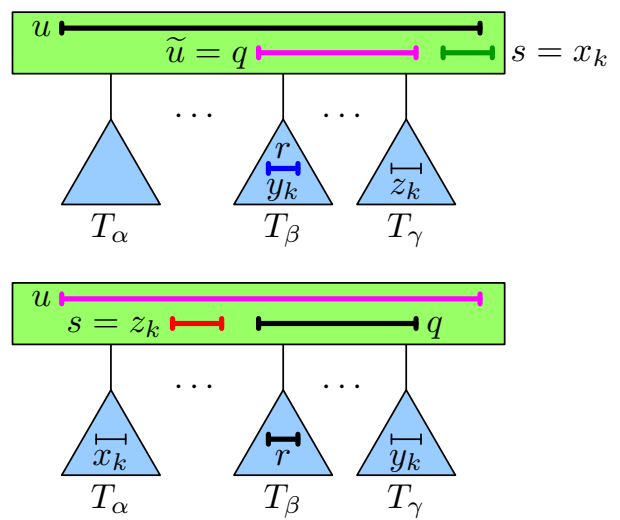

Figure 32: Proof of Lemma 29. On the left, the pre-drawn intervals. On the right, their positions in the MPQ-tree. (a) Case 1. (b) Subcase 2A. (c) Subcase 2B.

We apply Sliding Lemma 15 to $I_{a}, I_{b}$ and $r$, and we get a pre-drawn interval $s \notin P(a)$ covering $r(u)$. This sliding is weaker that in Case 1: we know that $s$ is on the right of $a$, but we do not know its position with respect to $c$. We distinguish three subcases according to the relative positions of $q$ and $s$ in the Q-node.

Subcase 2A: $s$ is on the right of $q$. The situation is depicted in Fig. 32b. Let $x_{k}=s$ and $y_{k}=r$. If $\langle q\rangle^{\prime}$ is on the left of $\langle r\rangle^{\prime}$, let $z_{k}=q$. Otherwise, let $z_{k} \in c$ be a vertex non-adjacent to $r$, but possibly adjacent to $x_{k}$. Since in every extending representation $z_{k}$ is placed on the left of $y_{k}$, we can apply $k$-FAT Lemma 22 to $x_{k}, y_{k}$ and $z_{k}$, and get a subgraph $H_{k}$. If $\langle q\rangle^{\prime}$ is on the left of $\langle r\rangle^{\prime}$, then $H_{k}$ gives a $k$-FAT obstruction. If $\langle r\rangle^{\prime}$ covers $r(q)$, then $H_{k}$ together with $\widetilde{u}=q$ gives a $k$-FS obstruction.

Subcase 2B: $s$ is on the left of $q$. We choose $x_{k} \in a$ and $y_{k} \in c$ non-adjacent to $s$; such vertices exist because $s$ is between $a$ and $c$ in the Q-node. By $(k, \ell)$-CE Lemma 24, we get a $(k, \ell)$-CE obstruction for $x_{k}, y_{k}, z_{k}=s$ and $u$. Notice that we can construct a path $P_{y_{k}, z_{k}}$ from $y_{k}$ to $z_{k}$ avoiding $N\left[x_{k}\right]$ by applying Sliding Lemma 15 to $I_{a}, I_{c}$, and $q$. Thus $\ell=1$.

Subcase 2C: $\langle s\rangle^{\prime}$ intersects $\langle q\rangle^{\prime}$. Notice that $\langle s\rangle^{\prime}$ also intersects $\langle r\rangle^{\prime}$. Therefore, if $s \notin P(c)$, then it appears in the Q-node between $a$ and $c$. Let $z_{k}=s$; we get a $(k, 1)$ CE obstruction as follows. We choose $y_{k} \in c$ non-adjacent to $z_{k}$. By Lemma 10, there exists $x_{k} \in a$ non-adjacent to $q, y_{k}$, and $z_{k}$. By $(k, \ell)$-CE Lemma 24 , we get a $(k, 1)$-CE obstruction for $x_{k}, y_{k}, z_{k}$ and $u$ as illustrated in Fig. 33a; notice that the path $y_{k} q z_{k}$ avoids $N\left[x_{k}\right]$.

It remains to deal with the situation when $s \in P(c)$. Let $z_{k}=r$. If $\langle q\rangle^{\prime}$ intersects $\langle r\rangle^{\prime}$, let $y_{k} \in c$ be a vertex non-adjacent to $r$; otherwise let $y_{k}=q$. By Lemma 10, there exists 
(a)

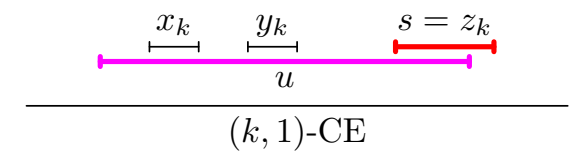

(c)

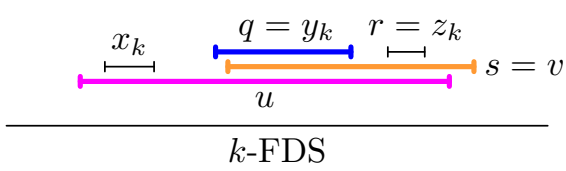

(b)

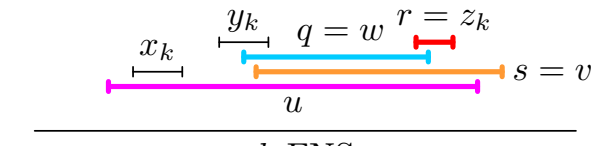

(d)

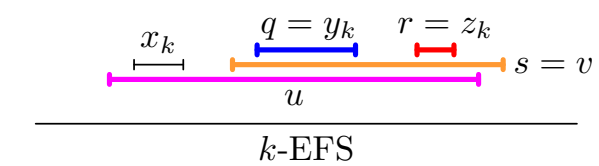

Figure 33: Four possible obstructions obtained in Subcase 2C of the proof of Lemma 29. (a) If $s \notin P(c)$, we get a $(k, 1)$-CE obstruction. (b) If $\langle q\rangle^{\prime}$ intersects $\langle r\rangle^{\prime}$, we get a $k$-FNS obstruction. Recall that the relative order of $\ell(q)$ and $\ell(s)$ does not matter. (c) If $\langle q\rangle^{\prime}$ is on the left of $\langle r\rangle^{\prime}$ and $\ell(q) \leqslant \ell(s)$, we get a $k$-FDS obstruction. (d) If $\langle q\rangle^{\prime}$ is on the left of $\langle r\rangle^{\prime}$ and $\ell(s)<\ell(q)$, we get a $k$-EFS obstruction.

$x_{k} \in a$ non-adjacent to $q, y_{k}$, and $z_{k}$. In every extending representation, $\left\langle y_{k}\right\rangle$ is placed on the left of $\left\langle z_{k}\right\rangle^{\prime}$, and $\left\langle x_{k}\right\rangle$ is placed on the left of $\left\langle y_{k}\right\rangle$. Therefore, by $k$-FAT Lemma 22, we get a subgraph $H_{k}$ of a $k$-FAT obstruction. Together with $u, v=s, w=q\left(\right.$ for $\left.y_{k} \neq q\right)$, or possibly some of them, we get a $k$-FDS, $k$-EFS, or $k$-FNS obstruction; see Fig. $33 \mathrm{~b}$, c, and $\mathrm{d}$.

The case where $P(b) \subsetneq P(c)$ is addressed in Lemmas 31 and 32. First, we need an auxiliary result.

Lemma 30. If $I_{a}$ is on the left of $I_{c}$ and $P(b) \subsetneq P(c)$, there exist $q \in P(c) \backslash P(b)$ and $r \in P(b) \backslash P(a)$ such that $\langle q\rangle^{\prime}$ is on the right of $I_{a}$ and on the left of $I_{b}$, and $\langle r\rangle^{\prime}$ is on the right of $I_{a}$, containing $I_{c}$ and $I_{b}$. Without loss of generality, $\langle q\rangle^{\prime}$ covers $\ell(r)$.

Proof. The proof is depicted in Fig. 34. Clearly, there exists $q \in P(c) \backslash P(b)$. Due to the structure of the Q-node, we also have that $q \notin P(a)$. Therefore, $\langle q\rangle^{\prime}$ is between $I_{a}$ and $I_{b}$.

Next, we argue that there exists $r \in P(b) \backslash P(a)$. For contradiction, assume that $P(b) \subsetneq P(a)$. Let $v \in P^{\mapsto}(b)$; notice that $v$ contains $I_{a}$ and $I_{c}$. By the flipped version of Sliding Lemma 15 applied to $I_{c}, I_{b}$ and $q$, there exists a path consisting of pre-drawn intervals not contained in $P(b)$ from $q$ to $z$, where $\langle z\rangle^{\prime}$ covers $\ell(v)$. At least one interval of this path intersects $I_{a}$, so it belongs to $P(a)$. This contradicts the fact that $b$ is between $a$ and $c$ in the Q-node. Hence, there exists $r \in P(b) \backslash P(a)$.
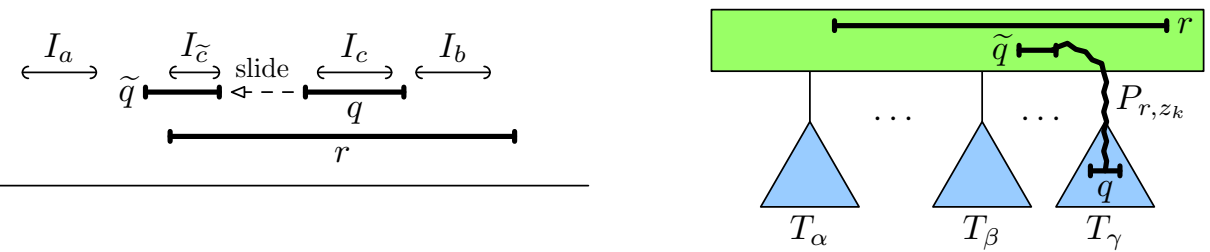

Figure 34: Proof of Lemma 30. On the left, the pre-drawn intervals. On the right, their positions in the MPQ-tree. 

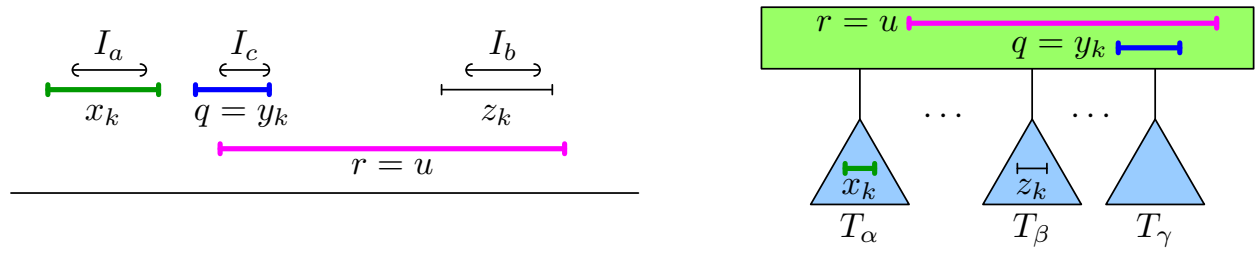

Figure 35: Proof of Lemma 31. We derive that $\left\langle x_{k}\right\rangle^{\prime}$ is on the left of $\langle q\rangle^{\prime}$, which gives a $k$-FS obstruction based on the positions in the Q-node.

We choose $r$ having rightmost left endpoint. Clearly, $\langle r\rangle^{\prime}$ is on the right of $I_{a}$, and contains $I_{b}$ and $I_{c}$. Suppose that $\ell(r)<\ell(q)$. Since $r$ has rightmost left endpoint among all intervals in $P(b) \backslash P(a)$, and every interval in $P(a)$ has its left endpoint more to the left, we obtain that $r \in P^{\mapsto}(b)$. Therefore, we can apply the flipped version of Sliding Lemma 15 to $I_{c}, I_{b}$ and $q$. We get a pre-drawn interval $\widetilde{q} \notin P(b)$ covering $\ell(r)$, and a path $P_{q, \widetilde{q}}$ from $q$ to $\widetilde{q}$ whose vertices are not in $b$. Therefore, $\widetilde{q}$ is on the right of $b$ in the Q-node. Let $\widetilde{c}$ be a maximal clique containing $\widetilde{q}$. Since $I_{\widetilde{c}}$ is contained in $\widetilde{q}$, it is between $I_{a}$ and $I_{b}$. Therefore, we can work with $\widetilde{q}$ and $\widetilde{c}$ instead of $q$ and $c$. Thus we can assume that $\langle q\rangle^{\prime}$ covers $\ell(r)$.

For $P(b) \subsetneq P(c)$, we distinguish two cases.

Lemma 31. If $I_{a}$ is on the left of $I_{c}, P(b) \subsetneq P(c)$, and $P(a) \backslash P(c) \neq \emptyset$, then $G$ and $\mathcal{R}^{\prime}$ contain a $k$-FS obstruction.

Proof. The proof is illustrated in Fig. 35. By Lemma 30, there exist $q \in P(c) \backslash P(b)$ and $r \in P(b) \backslash P(a)$ such that $\langle q\rangle^{\prime}$ covers $\ell(r)$. Let $x_{k} \in P(a) \backslash P(c)$ and $y_{k}=q$. Then $\left\langle x_{k}\right\rangle^{\prime}$ is on the left of $I_{c}$, and therefore also on the left of $I_{b}$. Thus $x_{k} \notin P(b)$. We infer that $x_{k}$ is on the left of $b$ in the Q-node, so it is non-adjacent to $y_{k}$. In consequence, $\left\langle x_{k}\right\rangle^{\prime}$ is on the left of $\left\langle y_{k}\right\rangle^{\prime}$. Let $z_{k} \in b$ be a vertex non-adjacent to $y_{k}$.

If $z_{k}$ is adjacent to $x_{k}$, we get a 1-FS obstruction. Otherwise, in every extending representation, $\left\langle z_{k}\right\rangle$ is to the right of $\left\langle y_{k}\right\rangle^{\prime}$. By $k$-FAT Lemma 22, we get a subgraph $H_{k}$ of a $k$-FAT obstruction. Together with $u=r$, this leads to a $k$-FS obstruction.

Lemma 32. If $I_{a}$ is on the left of $I_{c}, P(b) \subsetneq P(c)$ and $P(a) \subsetneq P(c)$, then $G$ and $\mathcal{R}^{\prime}$ contain a $(k, 1)-C E, k-F B, k-B I, k-F D S$, or $k$-EFDS obstruction.

Proof. Let $p \in P^{\leftrightarrow}(a)$, and $q$ be the vertex from Lemma 30. By applying Sliding Lemma 15 to $I_{a}, I_{c}$ and $q$, we get a pre-drawn interval $s \notin P(a)$ covering $r(p)$, and a path $P_{q, s}$ of intervals not in $P(a)$, so $s$ appears on the right of $a$ in the Q-node. Similarly as in Case 2 of the proof of Lemma 29, we distinguish three cases according to the relative positions of $s$ and $q$ in the Q-node; see Fig 36.

Case 1: $s$ is on the left of $q$. By Lemma 10, there exists $x_{k} \in a$ non-adjacent to all vertices of $P_{q, s}$, in particular non-adjacent to $s$ and $q$. Let $y_{k}=q, z_{k}=s$, and $u=p$. Clearly, $z_{k}$ is between $x_{k}$ and $y_{k}$ in the Q-node. By $(k, \ell)$-CE Lemma 24 and the existence of $P_{y_{k}, z_{k}}$, we get a $(k, 1)$-CE obstruction. Notice that $\left\langle y_{k}\right\rangle^{\prime}$ can be made free; see Fig. 36a. 
(a)

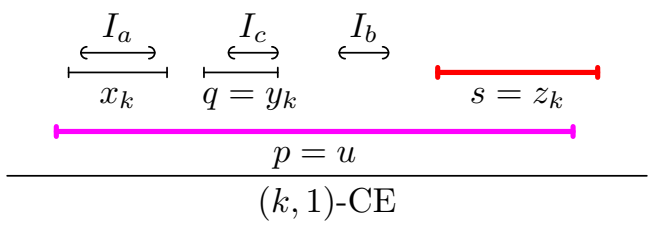

(b)

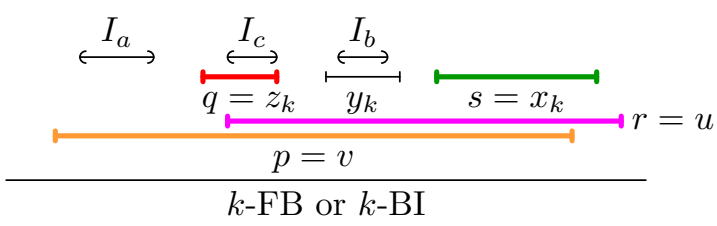

(c)

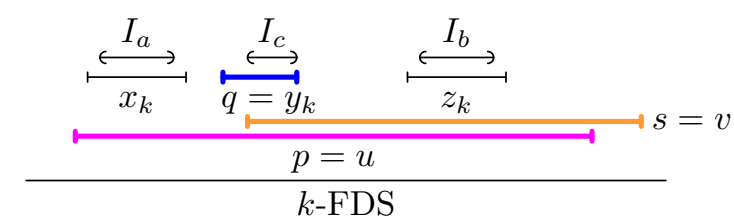

(d)

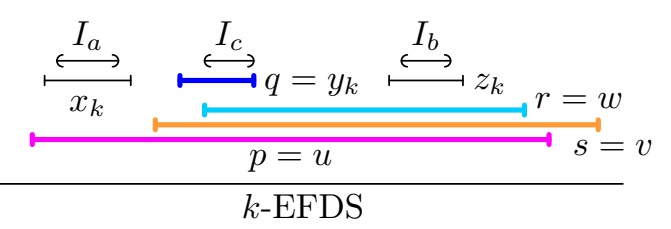

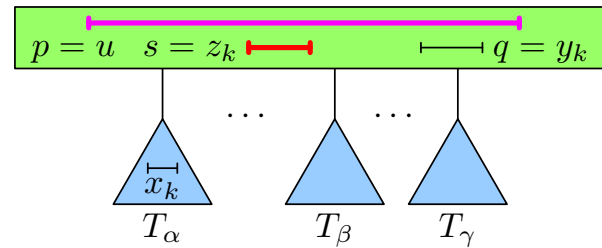
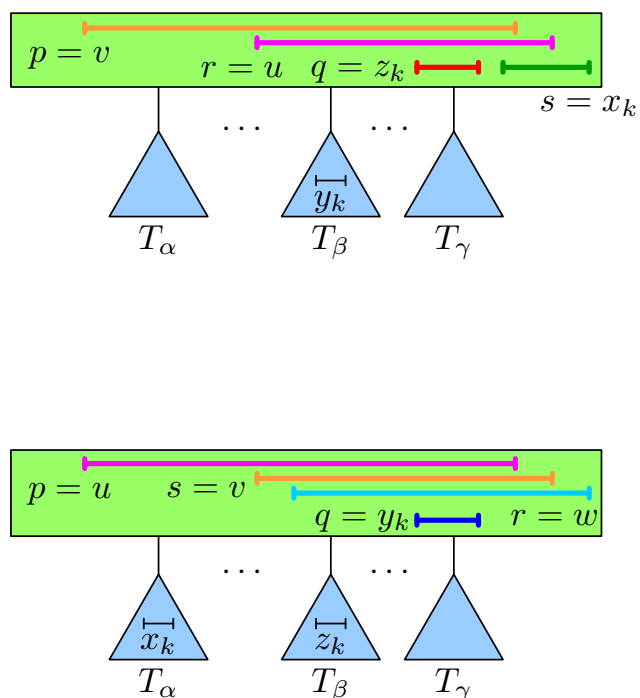

Figure 36: Proof of Lemma 32. On the left, the pre-drawn intervals. On the right, their positions in the MPQ-tree. (a) Case 1. (b) Case 2. (c) Case 3, if $\ell\left(y_{k}\right) \leqslant \ell(s)$. (d) Case 3 , if $\ell\left(y_{k}\right)>\ell(s)$.

Case 2: $s$ is on the right of $q$. Since $q$ is between $b$ and $s$ in the Q-node, we get that $s \notin P(b)$. Let $x_{k}=s, z_{k}=q$, and $u=r$, where $r$ is the vertex from Lemma 30. There exists $y_{k} \in b$ non-adjacent to $z_{k}$ and, by the structure of the Q-node, also non-adjacent to $x_{k}$. Since $y_{k}$ is adjacent to $p$ and $r,\left\langle y_{k}\right\rangle$ is between $\left\langle x_{k}\right\rangle^{\prime}$ and $\left\langle z_{k}\right\rangle^{\prime}$ in every extending representation. By $k$-FAT Lemma 22 , we get a subgraph $H_{k}$ of a $k$-FAT obstruction. If $r(r) \leqslant r\left(x_{k}\right)$, together with $u$, we obtain a $k$-FB or a $k$-BI obstruction. If $r(r)>r\left(x_{k}\right)$, together with $u$ and $v=p$, we obtain a $k$-BI obstruction; see Fig. 36b.

Case 3: $\langle s\rangle^{\prime}$ intersects $\langle q\rangle^{\prime}$. Since $s$ contains $I_{b}$, it belongs to $P(b)$. Let $y_{k}=q, u=p$, $v=s$, and $w=r$; we note that possibly $s=r$. By Lemma 10, there exists $x_{k} \in a$ non-adjacent to $y_{k}, v$, and $w$. Since $x_{k}$ is adjacent to $u$, then $\left\langle x_{k}\right\rangle$ is on the left of $\left\langle y_{k}\right\rangle^{\prime}$ in every extending representation. Finally, there exists $z_{k} \in b$ non-adjacent to $y_{k}$. Since $z_{k}$ is adjacent to $u, v$, and $w$, we have that $\left\langle z_{k}\right\rangle$ is on the right of $\left\langle y_{k}\right\rangle^{\prime}$ in every extending representation.

Since $z_{k}$ is between $x_{k}$ and $y_{k}$ in the Q-node, we can apply $k$-FAT Lemma 22, which gives a subgraph $H_{k}$ of a $k$-FAT obstruction. If $\ell\left(y_{k}\right) \leqslant \ell(v)$, together with $u$ and $v$, we obtain a $k$-FDS obstruction; see Fig. 36c. If $\ell\left(y_{k}\right)>\ell(v)$, together with $u, v$, and $w$, we 
get a $k$-EFDS obstruction; see Fig. 36d.

In summary, we conclude:

Lemma 33 (The Q-node, Three Subtrees). If the three cliques creating the obstruction belong to three different subtrees, then $G$ and $\mathcal{R}^{\prime}$ contain a $k$-FAT, $k$-BI $(k \leqslant 2), k-F S, k$ $E F S, k-F B, k-E F B, k-F D S, k-E F D S, k-F N S$, or $(k, \ell)-C E$ obstruction (either $k=\ell=2$, or $k \geqslant \ell=1$ ).

Proof. For an overview, see the diagram in Fig. 28. The proof follows from Lemmas 2632.

\section{Proofs of the Main Results}

Now, we are ready to put all results together to prove the main theorem. It states that a partial representation $\mathcal{R}^{\prime}$ of $G$ is extendible if and only if $G$ and $\mathcal{R}^{\prime}$ contain none of the obstructions described in Section 2.

Proof of Theorem 1. If $G$ and $\mathcal{R}^{\prime}$ contain one of the obstructions, they are non-extendible by Lemma 5 . It remains to prove the converse. If $G$ is not an interval graph, it contains an LB obstruction [30]. Otherwise, $G$ is an interval graph and there exists an MPQ-tree $T$ for it. By Lemma 12, we know that a partial representation $\mathcal{R}^{\prime}$ is extendible if and only if $T$ can be reordered according to $\triangleleft$. If it cannot be reordered, then the reordering algorithm fails in some node of $T$. If this reordering fails in a leaf, we get a 1-BI obstruction by Lemma 16. If it fails in a P-node, we get an SE, 1-BI, or 1-FAT obstruction by Lemma 19. And if it fails in a Q-node, we get one of the obstructions of Section 2 by Lemmas 20, 21, and 33 .

Next, we show that a partial representation $\mathcal{R}^{\prime}$ is extendible if and only if every quadruple of pre-drawn intervals is extendible by itself.

Proof of Corollary 2. The result follows from the fact that all the obstructions of Theorem 1 contain at most four pre-drawn intervals.

Concerning the certifying algorithm, we first show that $k$-FAT obstructions can be found in linear time:

Lemma 34. Suppose that the assumptions of $k$-FAT Lemma 22 are satisfied. Then we can find a $k$-FAT obstruction in time $\mathcal{O}(n+m)$.

Proof. Since the proof of $k$-FAT Lemma 22 is constructive, the algorithm follows it. Let $Q$ be the Q-node. We search the graph $G[Q] \backslash N\left[y_{k}\right]$ from $x_{k}$ to compute $C\left(x_{k}\right)$, and test whether $z_{k}$ belongs to it. If it does, the algorithm stops and outputs 1-FAT. Otherwise, we compute $W_{k}$, choose $t_{k}$, and store it together with $P_{k}$. We choose $x_{k-1}$ as in the the proof; if $s_{i}(Q)=s_{t_{k}}(Q)$, then either $s_{x_{k-1}}^{\leftarrow}(Q)=s_{i+1}(Q)$, or $x_{k-1}$ belongs to sections of $T_{i+1}$. Then we apply the rest of the algorithm recursively. It is important that then we 
can remove $C\left(x_{k}\right)$ and $W_{k}$ from the graph because they are not used in the remainder of the obstruction.

Since the algorithm searches each vertex and edge of $G[Q] \backslash N\left[y_{k}\right]$ at most once when computing $C\left(x_{j}\right)$, we obtain that the algorithm runs in time $\mathcal{O}(n+m)$.

Similarly, a $(k, \ell)$-CE obstruction can be obtained from $(k, \ell)$-CE Lemma 24 in time $\mathcal{O}(n+m)$. Since obstructions are built constructively, we get a linear-time certifying algorithm for the partial representation extension problem:

Proof of Corollary 3. We can assume that $G$ is an interval graph; otherwise we can find an LB obstruction in time $\mathcal{O}(n+m)$ using [32]. Each interval graph has $\mathcal{O}(n)$ maximal cliques of total size $\mathcal{O}(n+m)$, and that they can be found in linear time [36]. We compute the MPQ-tree $T$ in time $\mathcal{O}(n+m)$ using [28]. Next, we use the partial representation extension algorithm of [23] in time $\mathcal{O}(n+m)$, which either finds an extending representation, or finds an obstructed node which cannot be reordered according to $\triangleleft$. We distinguish three cases according to the distinct types of obstructed nodes.

Case 1: A leaf cannot be reordered. We output a 1-BI obstruction in time $\mathcal{O}(n)$, by searching the partial representation.

Case 2: A P-node P cannot be reordered. From the partial representation extension algorithm, we get directly a two-cycle, ensured by Lemma 17, and four maximal cliques $a, b, c$, and $d$ defining it. By Lemma 18, one of these maximal cliques can be omitted, and it can be clearly found in constant time. It remains to output an SE, 1-BI, or 1-FAT obstruction in time $\mathcal{O}(n+m)$, by following Lemma 19. For 1-BI and 1-FAT obstructions, we find a shortest path in $G[P] \backslash N\left[y_{k}\right]$ by searching the graph.

Case 3: A Q-node $Q$ cannot be reordered. From the partial representation extension algorithm, we get four maximal cliques defining the obstruction and, by following Lemma 20, we can reduce it to at most three maximal cliques. An SE obstruction can be computed in time $\mathcal{O}(n+m)$. If three maximal cliques are contained in two subtrees, we follow Lemma 21 and output one of the obstructions in time $\mathcal{O}(n+m)$.

If three maximal cliques belong to three different subtrees, we follow the structure of the proof of Lemma 33. In all cases, we derive some vertices somehow placed in the Q-node and some pre-drawn intervals, which can be easily done in time $\mathcal{O}(n+m)$. Next, we either apply $k$-FAT Lemma, or $(k, \ell)$-CE Lemma to construct the obstruction, which can be done in time $\mathcal{O}(n+m)$ by Lemma 34 .

\section{Conclusions}

In this paper, we have described the minimal obstructions that make a partial interval representation non-extendible. There are three main points following from the proof:

1. Minimal obstructions for the partial representation extension problem are much more complicated than minimal forbidden induced subgraphs of interval graphs, characterized by Lekkerkerker and Boland [30]. 
2. Nevertheless, it is possible to describe these obstructions using structural results derived in [23] and in this paper. We show that almost all of these obstructions consist of three intervals $x_{k}, y_{k}$ and $z_{k}$ that are forced by the partial representation to be drawn in an incorrect left-to-right order. This incorrect placement leads to the complex zig-zag structure of a $k$-FAT obstruction.

3. The structure of the sections of a Q-node $Q$ can be very intricate. Suppose that we contract in $G[Q]$ the sections of each subtree $T_{i}$ into one vertex. Then we get an interval graph which has a unique interval representation up to flipping the real line. Such interval graphs have been extensively studied, see for instance $[16,12,34]$. Therefore, our structural results needed to find minimal obstructions may be of independent interest.

Structural Open Problems. The first open problem we propose is a characterization of minimal obstructions for other graph classes. We select those classes for which polynomial-time algorithms are known [8, 20, 21]. Circle graphs (CIRCLE) are intersection graphs of chords of a circle. Function graphs (FUN) are intersection graphs of continuous functions $f:[0,1] \rightarrow \mathbb{R}$, and permutation graphs (PERM) are function graphs which can be represented by linear functions. Proper interval graphs (PROPER INT) are intersection graphs of closed intervals in which no interval is a proper subset of another interval. Unit interval graphs (UNIT INT) are intersection graphs of closed intervals of length one.

Problem 35. What are the minimal obstructions for partial representation extension of the classes CIRCLE, FUN, PERM, PROPER INT, and UNIT INT?

The second open problem involves a generalization of partial representations called bounded representations $[2,21,37,38]$. Suppose that a graph $G$ is given together with two closed intervals $L_{v}$ and $R_{v}$ for every vertex $v \in V(G)$. A bounded representation of $G$ is a representation such that $\ell(v) \in L_{v}$ and $r(v) \in R_{v}$ for every vertex $v \in V(G)$. We call bounds solvable if and only if there exists a bounded representation. This generalizes partial representations: we can use singletons $L_{v}$ and $R_{v}$ for pre-drawn intervals and put them equal $\mathbb{R}$ for the others.

Problem 36. What are minimal obstructions making bounds for interval graphs unsolvable?

Algorithmic Open Problems. We have described a linear-time certifying algorithm that can find one of the minimal obstructions in a non-extendible partial representation. There are several related computational problems, suggested by Jan Kratochvíl, for which the complexity is open:

Problem 37. What is the computational complexity of the problem of testing whether a given minimal obstruction is contained in $G$ and $\mathcal{R}^{\prime}$ ? 
Since a minimal obstruction contains at most four pre-drawn intervals, we can test over all subsets of at most four pre-drawn intervals whether they form an obstruction (say, by freeing the rest of them and testing whether the modified partial representation is extendible). If $k$ is fixed, we can test whether the subgraph of a given obstruction is contained in $G$. Given a triple $x_{k}, y_{k}$ and $z_{k}$ forming a $k$-FAT obstruction, the proof of $\mathrm{k}$-FAT Lemma 22 and the algorithm of Lemma 34 constructs it while minimizing $k$. The approach needs to be changed to check whether they also form an $\ell$-FAT obstruction, for $\ell>k$.

The next problem generalizes the partial representation extension problem.

Problem 38. What is the computational complexity of testing whether at most $\ell$ predrawn intervals can be freed to make a partial representation $\mathcal{R}^{\prime}$ extendible?

Similar problems are usually NP-complete. On the other hand, we propose the following reformulation which might lead to a polynomial-time algorithm. Every minimal obstruction contains at most four pre-drawn intervals. Let $P$ be the set of pre-drawn intervals, and let $\mathcal{S}$ consist of all subsets of $P$ of size at most four which form an obstruction. We can clearly compute $\mathcal{S}$ in polynomial time. Then the problem above is equivalent to finding a minimal hitting set of $P$ and $\mathcal{S}$. This problem is in general NP-complete, but the extra structure given by the MPQ-tree might make it tractable.

Problem 39. What is the complexity of testing whether it is possible to remove at most $\ell$ vertices from an interval graph $G$ to make a partial representation extendible $\mathcal{R}^{\prime}$ ?

This problem is fundamentally different from Problem 38, in which the partial representation $\mathcal{R}^{\prime}$ is modified. In this problem, we modify the graph $G$ itself, changing its structure. When we remove a pre-drawn vertex, we also remove its pre-drawn interval from the partial representation. We note that the assumption that $G$ is an interval graph is important. For general graphs $G$, the problem is known to be NP-complete even when $\mathcal{R}^{\prime}=\emptyset[31]$.

\section{Acknowledgements}

We would like to thank Jaroslav Nešetřil for suggesting us the problem of minimal obstructions for partial representation extension, and to an anonymous reviewer for helpful suggestions concerning writing of this paper, in particular the properties of $\triangleleft$.

\section{References}

[1] P. Angelini, G. Di Battista, F. Frati, V. Jelínek, J. Kratochvíl, M. Patrignani, and I. Rutter. Testing planarity of partially embedded graphs. ACM Trans. Algorithms, 11(4):32:1-32:42, 2015.

[2] M. Balko, P. Klavík, and Y. Otachi. Bounded representations of interval and proper interval graphs. In Algorithms and Computation, ISAAC 2013, volume 8283 of Lecture Notes in Computer Science, pages 535-546, 2013. 
[3] J. Bang-Jensen, J. Huang, and X. Zhu. Completing orientations of partially oriented graphs. J. Graph Theory, 87(3):285-304, 2018.

[4] S. Benzer. On the topology of the genetic fine structure. Proc. Nat. Acad. Sci. U.S.A., 45:1607-1620, 1959.

[5] T. Bläsius and I. Rutter. Simultaneous pq-ordering with applications to constrained embedding problems. ACM Trans. Algorithms, 12(2):16:1-16:46, 2015.

[6] K. Booth and G. Lueker. Testing for the consecutive ones property, interval graphs, and planarity using PQ-tree algorithms. J. Comput. System Sci., 13:335-379, 1976.

[7] S. Chaplick, P. Dorbec, J. Kratochvíl, M. Montassier, and J. Stacho. Contact representations of planar graphs: Extending a partial representation is hard. In GraphTheoretic Concepts in Computer Science, WG 2014, volume 8747 of Lecture Notes in Computer Science, pages 139-151. 2014.

[8] S. Chaplick, R. Fulek, and P. Klavík. Extending partial representations of circle graphs. In Graph Drawing, GD 2013, volume 8242 of Lecture Notes in Computer Science, pages 131-142, 2013.

[9] S. Chaplick, G. Guśpiel, G. Gutowski, T. Krawczyk, and G. Liotta. The partial visibility representation extension problem. Algorithmica, 80(8):2286-2323, 2018.

[10] C. J. Colbourn and K. S. Booth. Linear times automorphism algorithms for trees, interval graphs, and planar graphs. SIAM J. Comput., 10(1):203-225, 1981.

[11] D. G. Corneil, S. Olariu, and L. Stewart. The LBFS structure and recognition of interval graphs. SIAM J. Discrete Math., 23(4):1905-1953, 2009.

[12] P. C. Fishburn. A characterization of uniquely representable interval graphs. Discrete Appl. Math., 12:191-194, 1985.

[13] P. C. Fishburn. Interval graphs and interval orders. Discrete Math., 55(2):135-149, 1985.

[14] D. R. Fulkerson and O. A. Gross. Incidence matrices and interval graphs. Pacific J. Math., 15:835-855, 1965.

[15] G. Hajós. Über eine Art von Graphen. Internat. Math. News, 11:65, 1957.

[16] P. Hanlon. Counting interval graphs. Trans. Amer. Math. Soc., 272(2):383-426, 1982.

[17] V. Jelínek, J. Kratochvíl, and I. Rutter. A kuratowski-type theorem for planarity of partially embedded graphs. Comput. Geom., 46(4):466-492, 2013.

[18] R. M. Karp. Mapping the genome: Some combinatorial problems arising in molecular biology. In Proceedings of the Twenty-fifth Annual ACM Symposium on Theory of Computing, STOC '93, pages 278-285, 1993.

[19] D. G. Kendall. Incidence matrices, interval graphs and seriation in archaeology. Pacific J. Math., 28(3):565-570, 1969. 
[20] P. Klavík, J. Kratochvíl, T. Krawczyk, and B. Walczak. Extending partial representations of function graphs and permutation graphs. In Algorithms, ESA 2012, volume 7501 of Lecture Notes in Computer Science, pages 671-682, 2012.

[21] P. Klavík, J. Kratochvíl, Y. Otachi, I. Rutter, T. Saitoh, M. Saumell, and T. Vyskočil. Extending partial representations of proper and unit interval graphs. Algorithmica, 77(4):1071-1104, 2017.

[22] P. Klavík, J. Kratochvíl, Y. Otachi, and T. Saitoh. Extending partial representations of subclasses of chordal graphs. Theoret. Comput. Sci., 576:85-101, 2015.

[23] P. Klavík, J. Kratochvíl, Y. Otachi, T. Saitoh, and T. Vyskočil. Extending partial representations of interval graphs. Algorithmica, 78(3):945-967, 2017.

[24] P. Klavík, J. Kratochvíl, and T. Vyskočil. Extending partial representations of interval graphs. In Theory and Applications of Models of Computation, TAMC 2011, volume 6648 of Lecture Notes in Computer Science, pages 276-285, 2011.

[25] P. Klavík, Y. Otachi, and J. Šejnoha. On the classes of interval graphs of limited nesting and count of lengths. In Algorithms and Computation, ISAAC 2016, volume 64 of Leibniz International Proceedings in Informatics (LIPIcs), pages 45:1-45:13, 2016.

[26] P. Klavík, Y. Otachi, and J. Šejnoha. On the classes of interval graphs of limited nesting and count of lengths. Algorithmica, 2018. https://doi.org/10.1007/s00453018-0481-y

[27] P. Klavík and M. Saumell. Minimal obstructions for partial representations of interval graphs. In Algorithms and Computation, ISAAC 2014, volume 8889 of Lecture Notes in Computer Science, pages 401-413, 2014.

[28] N. Korte and R. Möhring. An incremental linear-time algorithm for recognizing interval graphs. SIAM J. Comput., 18(1):68-81, 1989.

[29] T. Krawczyk and B. Walczak. Extending partial representations of trapezoid graphs. In Graph-Theoretic Concepts in Computer Science, WG 2017, volume 10520 of Lecture Notes in Computer Science, pages 358-371. 2017.

[30] C. Lekkerkerker and D. Boland. Representation of finite graphs by a set of intervals on the real line. Fund. Math., 51:45-64, 1962.

[31] J. M. Lewis and M. Yannakakis. The node-deletion problem for hereditary properties is NP-complete. J. Comput. System Sci., 20(2):219-230, 1980.

[32] N. Lindzey and R. M. McConnell. Linear-time algorithms for finding tucker submatrices and lekkerkerker-boland subgraphs. SIAM J. Discrete Math., 30(1):43-69, 2016.

[33] M. Patrignani. On extending a partial straight-line drawing. Int. J. Found. Comput. Sci., 17(5):1061-1070, 2006.

[34] I. Pe'er and R. Shamir. Realizing interval graphs with size and distance constraints. SIAM J. Discrete Math., 10(4):662-687, 1997.

[35] F. S. Roberts. Discrete Mathematical Models, with Applications to Social, Biological, and Environmental Problems. Prentice-Hall, Englewood Cliffs, 1976. 
[36] D. J. Rose, R. E. Tarjan, and G. S. Lueker. Algorithmic aspects of vertex elimination on graphs. SIAM J. Comput., 5(2):266-283, 1976.

[37] F. J. Soulignac. Bounded, minimal, and short representations of unit interval and unit circular-arc graphs. Chapter I: theory. J. Graph Algorithms Appl., 21(4):455489, 2017.

[38] F. J. Soulignac. Bounded, minimal, and short representations of unit interval and unit circular-arc graphs. Chapter II: algorithms. J. Graph Algorithms Appl., 21(4):491$525,2017$.

[39] K. E. Stoffers. Scheduling of traffic lights-a new approach. Transportation Research, 2:199-234, 1968.

[40] P. Zeman. Extending partial representations of unit circular-arc graphs. arXiv:1706.00928 2017. 